\title{
Hybrid Renewable Energy System Based On Intelligent Optimization Techniques
}

By

Katheryn Donado Mercado

\section{MASTER THESIS}

Advisor

Dr. Christian G. Quintero M.

\author{
Barranquilla, Atlántico, Colombia \\ June 2017
}




\section{Hybrid Renewable Energy System Based On Intelligent Optimization Techniques}

A dissertation presented to the Universidad del Norte in partial fulfillment of the requirements for the degree of MASTER OF SCIENCE

By

Katheryn Donado Mercado

Advisor:

Dr. Christian G. Quintero M.

Barranquilla, Atlántico, Colombia

June 2017 


\title{
ABSTRACT \\ Hybrid Renewable Energy System Based On Intelligent Optimization Techniques
}

\author{
By Katheryn Donado Mercado \\ Advisor: Dr. Christian G. Quintero M.
}

Renewable energy aims to generate more ecological and non-pollutant energy and for that, they are essential for sustainable development of the society. Generation with renewable energies must take into account the deficiencies and limitations that arise because of the intermittent capacity of power supply. Hybrid renewable energy systems based on solar and wind resources are the most promising energy generation power systems due to their complementarity to exploit natural resources; however, the installation of a hybrid solar-wind system is dependent of the weather conditions and that makes the dimension of the system complex.

An optimal design of the hybrid system to be implemented becomes critical in order to have the best cost-reliability ratio. Applying intelligent optimization techniques makes possible to find an optimal design in a suitable time. The literature shows that the most used optimization technique is genetic algorithms.

This master thesis, proposes to use intelligent technologies to optimally size a hybrid renewable energy system using solar and wind power. The proposed approach requires the system configuration, providing or not grid connection, 
battery storage and the optimization approaches. Experimental results and conclusions emphasizing the advantages of intelligence optimization techniques for the improvement of hybrid renewable energy systems are presented. 


\section{Acknowledgments}

To God for His infinite blessings. To my parents for their unconditional support, motivation and care. To my husband for making this process simpler with his love, patience and human warmth.

Special thanks to my advisor Christian G. Quintero M. for his guidance, constant support, impartial corrections and confidence; for encouraging me to continue at all times and give me his friendship.

My teachers Luis Torres, Juan Velez, Ingrid Oliveros, Cesar Orozco for allowing me to grow as a master's student. To Jamer Jimenez for his supervision, friendship and care.

To my companions for sharing this path full of challenges with me and make it easier and his unconditional help. To my relatives and those whom I did not name, thank you. 
To my God, parents, family and friends 


\section{General Contents}

PART I: INTRODUCTION AND RELATED WORK

Motivation, objectives, main contributions and an overview of general concepts used in this thesis dissertation.

A review of relevant related work used as reference and inspiration to develop the proposed approach.

PART II: PROPOSED APPROACH

General considerations and implementation of the proposed system for sizing hybrid renewable energy systems approach that seeks to provide broad decisions when designing optimal systems with intelligence techniques

\section{PART III: EXPERIMENTAL RESULTS AND CONCLUSIONS}

Analysis and discussion of the experimental results, conclusions and future research related to hybrid renewable energy systems based on intelligent systems. 


\section{Detailed Contents}

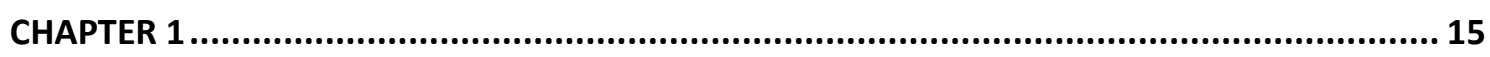

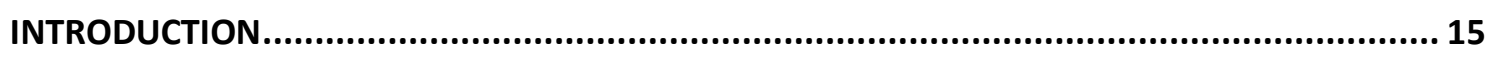

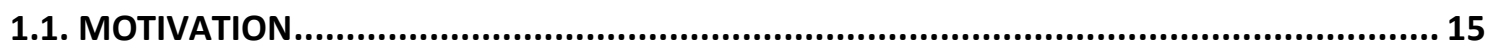

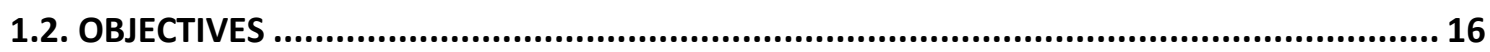

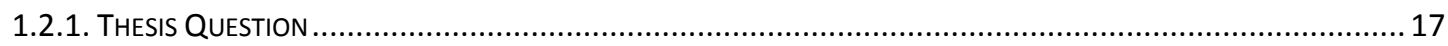

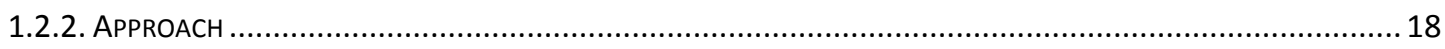

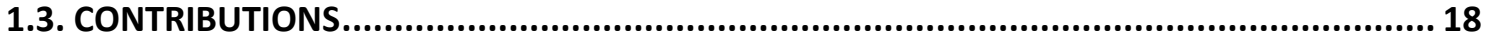

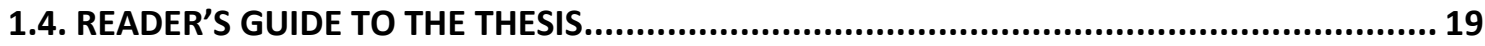

CHAPTER 2

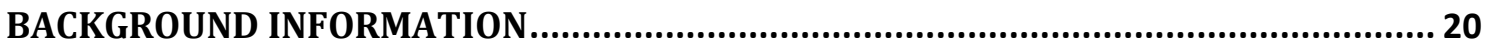

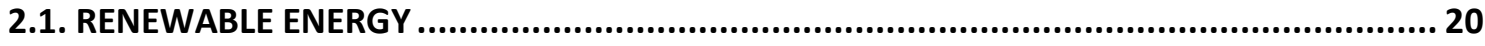

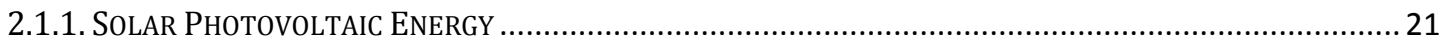

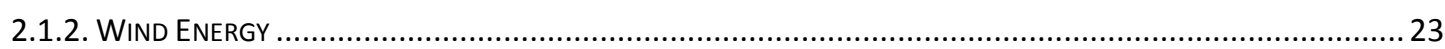

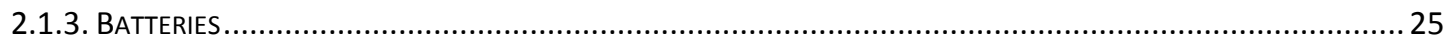

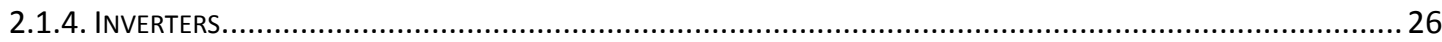

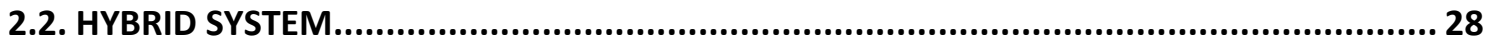

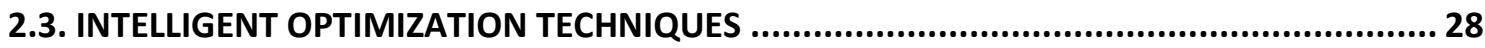

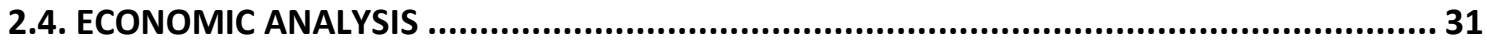

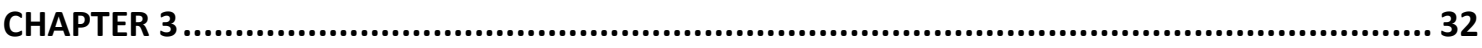

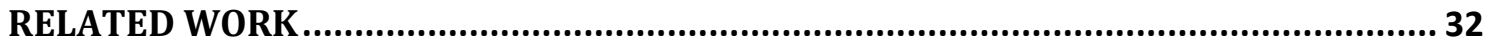

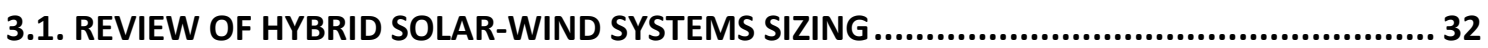

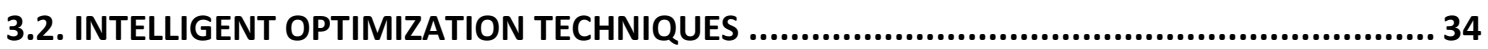

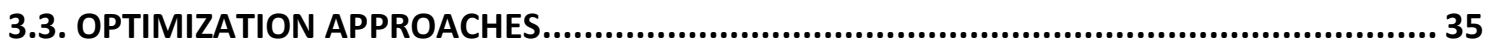

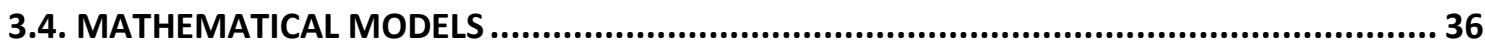

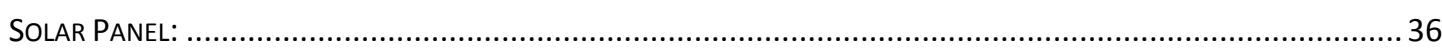

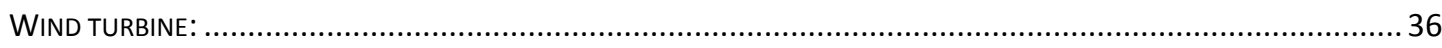




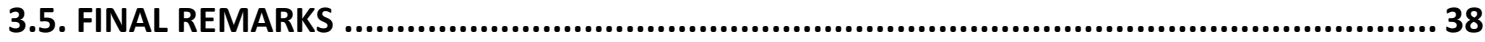

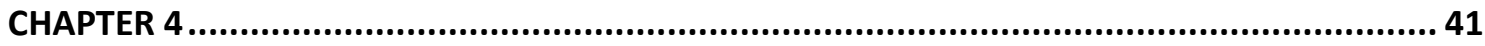

HYBRID RENEWABLE ENERGY SYSTEMS APPROACH .......................................... 41

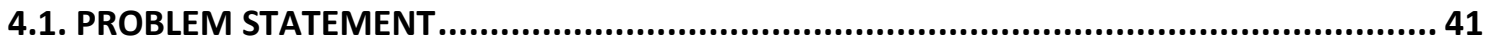

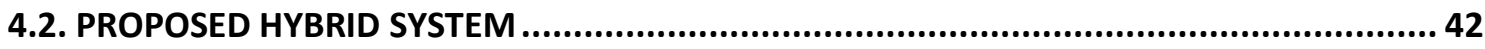

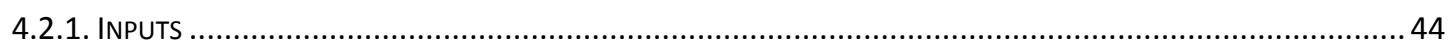

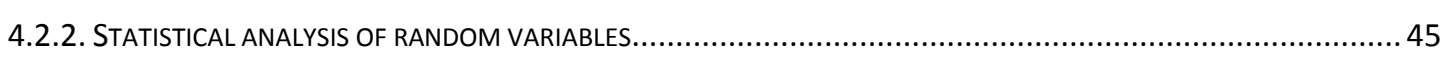

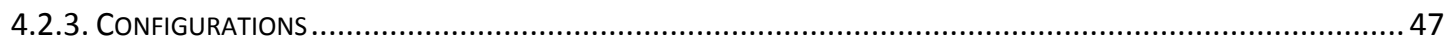

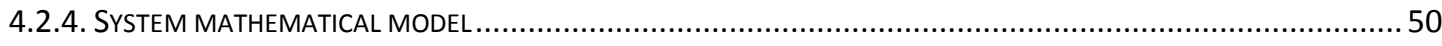

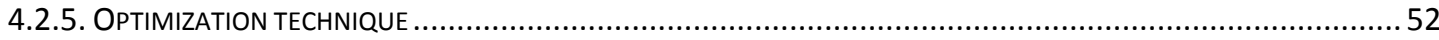

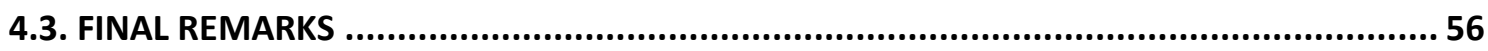

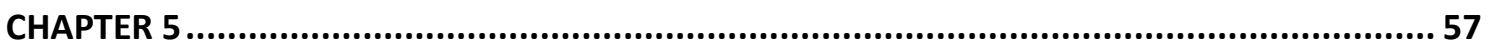

IMPLEMENTATION OF A SIZING APPROACH FOR HYBRID RENEWABLE ENERGY

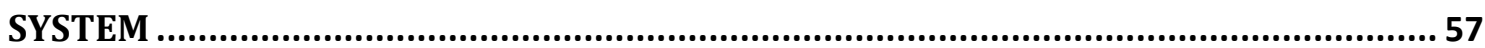

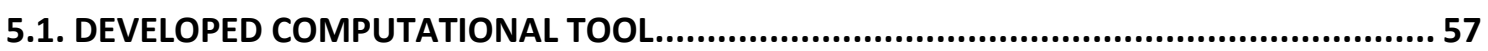

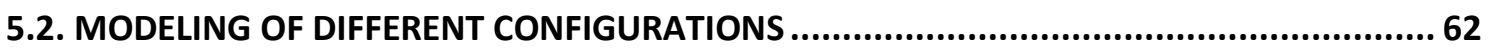

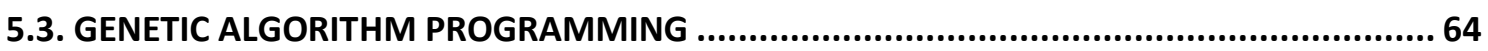

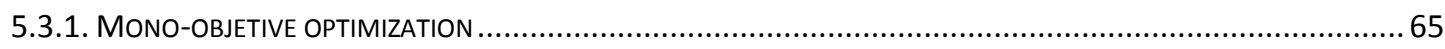

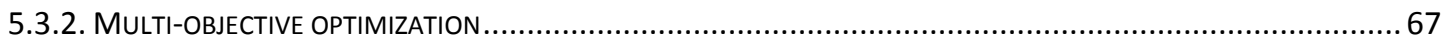

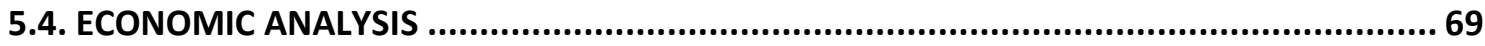

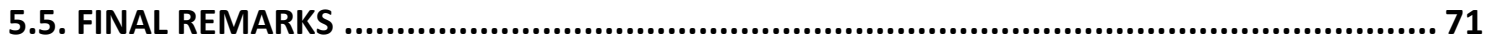

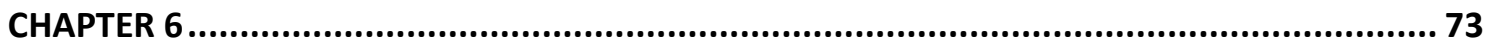

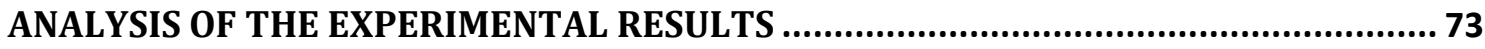

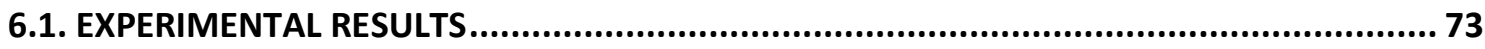

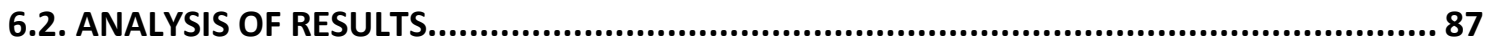

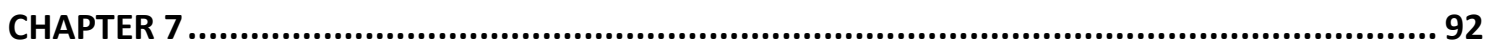

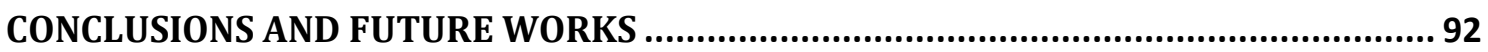


7.1. CONCLUSIONS

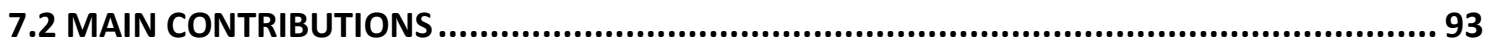

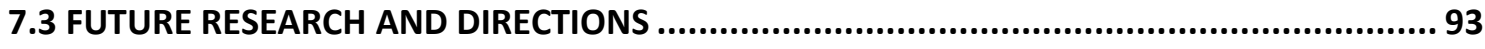

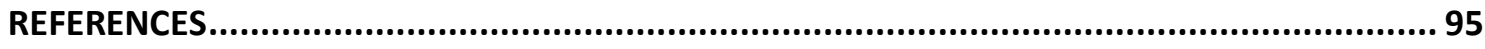

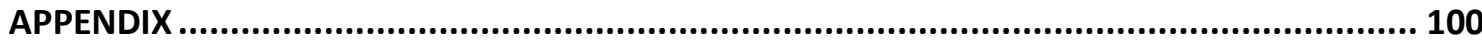




\section{List of Figures}

Figure 1. Natural energy currents on earth. Taken from (Twidell \& Weir, 2006)..21 Figure 2. Cell array composition with any virtually size. Taken by (Twidell \& Weir, 2006)

Figure 3. Main types of wind turbines. A) HAWTs, B) VAWTs. Taken by (Kalogirou, 2013)

Figure 4. Battery system connection with 16 batteries. 26

Figure 5. Connection of two inverters in parallel for hybrid systems 27

Figure 6. Comparison between intelligent optimization techniques used to optimize hybrid wind-solar systems.

Figure 7. Comparison between optimization approaches used to optimize hybrid systems.

Figure 8. Thesis context. 42

Figure 9. Schematic of the proposed hybrid renewable system 43

Figure 10. Steps for system implementation. 43

Figure 11. Inputs of the system 44

Figure 12. Wind speed and solar radiation of hour 9 with $50^{\text {th }}$ percentile in the left and $30^{\text {th }}$ percentile in the right image. Data from Barranquilla, Colombia. 46

Figure 13. Configuration operation: Grid-Connected with batteries 48

Figure 14. Configuration operation: Grid-connected with possibility to sale surplus energy . .48

Figure 15. Operation of the Off-grid configuration: Wind-Solar-batteries. 49

Figure 16. Operation of the Grid-connected configurationWind-Solar-Grid 49

Figure 17. General structure of the methodology 56

Figure 18. Selection of local currency in the developed system. 57

Figure 19. Inputs of the developed system. 58

Figure 20. Manual input displayed windows for solar panel, wind turbine and battery. 60

Figure 21. Graphical software interface . .61

Figure 22. Output with life cycle cost optimization approach selected. 62 
Figure 23. Interactive window showing the convergence of optimization .67

Figure 24. Interactive window showing the Pareto Front obtained ...........................69

Figure 25. Flow diagram in the system life cycle time. ................................................ 70

Figure 26. Load demand of user 1 ............................................................................. 74

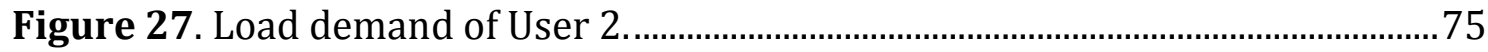

Figure 28. Result from HOMER for Case7 .......................................................................... 79

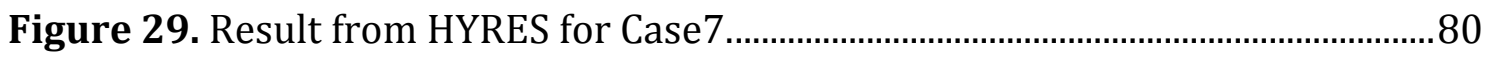

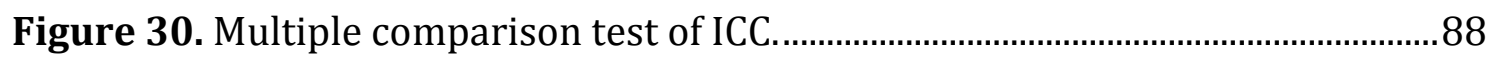

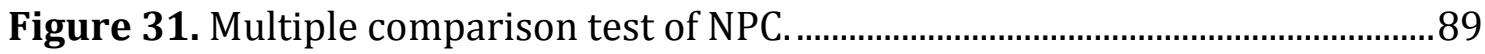

Figure 32. Multiple comparison test of Renewable Fraction. ..........................................89

Figure 33. Comparison of the results of mono-objective and multi-objective optimization with a) ICC, b)LCC, c)LPS, d)Reliability, e)Payback years approaches. 


\section{List of Tables}

Table 1. Studied mathematical model for solar panel.....................................................

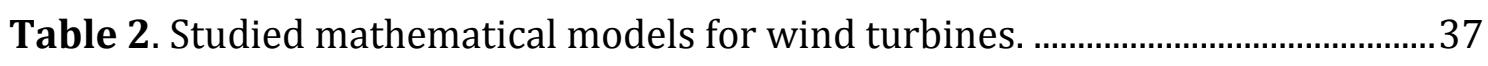

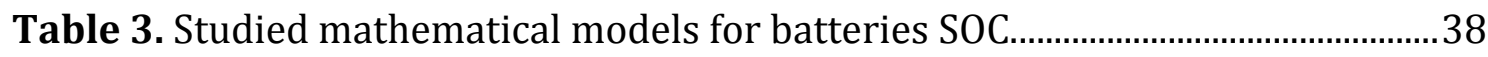

Table 4. Different cases for the comparison between HYRES and HOMER systems.

Table 5. Comparison between HOMER and HYRES systems......................................... 78

Table 6. Results of HYRES with mono-objective optimization ......................................84

Table 7. Results of HYRES with multi-objective optimization ......................................86

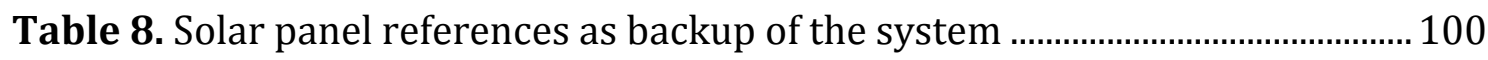

Table 10. Wind turbine references as backup of the system ...................................... 100

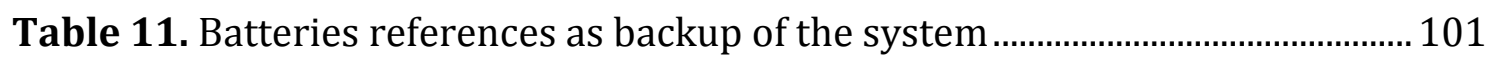




\section{PART I \\ INTRODUCTION AND \\ RELATED WORK}




\section{Chapter 1}

\section{Introduction}

This chapter introduces the work presented in this thesis. Specifically, the motivation in the research area, the pursued aims and the main contributions are briefly described. Finally, the chapter concludes with an overview of the structure and contents of the thesis.

\subsection{Motivation}

The cost of electricity is increasing due to its dependence on fossil fuels, the reduction of accessibility to its resources and the rise in energy consumption. In addition, greenhouse gas emissions are threatening natural ecosystems. On the other hand, renewable sources are unlimited and respectful of the environment. Amongst renewable energy, solar and wind energy systems are generally considered the most promising technologies to meet the consumption of electricity because of their accessibility and the omnipresent and free availability of their resources (Zhou, et al., 2010). However, systems using solar or wind resources must take into account the shortcomings and limitations that arise as a result of intermittent power supply capacity; in fact, the use of them is not valuable since the resource is not continuous. In this sense, it is evident the need to promote renewable energy generation systems to more robust, resistant, manageable and reliable systems, which generates great interest in this study.

In order to improve simple renewable energy drawbacks, the resources are integrated to construct hybrid system configurations. Such systems are characterized for the use of two or more energy sources, mainly renewables, in order to make them complementary, sustainable and economical. Studies conducted 
around the globe have demonstrated powerful effective of hybrid systems in satisfying a specific energy requirement.

Concerning the fact of making the use of renewable energy sources more efficient, an optimum design becomes critical, based on prediction of the renewable energy resources data. So that, there is a need to select and configure the optimal sizing of all components; in fact, over-sizing the system entails a high investment cost and other additional issues such as the area occupied by the system. Contrary to this, under-sizing the system may involve low investment costs but a high cost of operating limitations and therefore possible shortcomings in energy consumption. Hybrid systems may vary according to weather and restrictions due to the geographical area, limitations and conditions in the place where the system will be installed like available area for solar panels and wind turbines and grid service connection that may vary the configuration of the system as the quantity and type of elements. In addition, the optimization may be focused on low cost, high efficiency or both.

This research seeks to develop a system capable to adapt to the different environments: weather conditions, cost, reliability, available area $\left(\mathrm{m}^{2}\right)$, and configuration of the system (grid connected, off grid, storage system, ability to sale energy excess), technology of the elements to use (select references from a backup, set the technical parameters of the elements, optimize the best references from the backup), daily load consumption (hour by hour). In each case, an optimization approach can be selected and the other system limitations can be taken as constraints or a multi-objective optimization method can be used.

\subsection{0bjectives}

This work is focused on the use of intelligent optimization techniques to optimally size a hybrid system with renewable energy sources using solar photovoltaic and wind power. 
- Problem: Dimension a hybrid solar-wind system under weather, local, efficiency and economical restrictions.

- General Objective: Develop an intelligent energy hybrid power generation system with renewable energy sources to optimally size the configuration to be installed to satisfy electrical demand of the user with reliability parameters.

\section{- Goals:}

$\checkmark$ Characterize users and renewable energy sources to use in system.

$\checkmark$ Design and implement an intelligent system to optimally size the hybrid renewable energy system configuration considering previously characterized variables and constraints.

$\checkmark$ Evaluate the performance of the developed intelligent hybrid renewable energy system applied in several case studies.

The first objective refers to the mathematical models of the energy sources that have been selected as well as the technologies to be used, on the other hand, in terms of users, it is done in order to know the restrictions as available areas, energy demand, type of system to be implemented and economic conditions. The second objective looks to design and implement, in computational context, a system that allows with established input variables, to optimize the sizing of the hybrid system to be installed in each case study. Finally, it is sought to evaluate the behavior of the dimensioning system implemented in this thesis by comparing its answers with the HOMER software.

\subsubsection{Thesis Question}

The principal question addressed in this dissertation is:

¿Which is the optimal size of a hybrid generation system (solar photovoltaicwind based) to satisfy an electrical demand in a specific location and ensure system reliability and power supply set? 


\subsubsection{Approach}

In this research is proposed the design of a system capable to size a hybrid renewable energy system with solar panels and wind turbines for a load demand and restrictions set by user. This approach incorporates an intelligent optimization technique selected from the literature reviews in order to guarantee the best configuration in each case, also includes two types of reliability (annually and daily) in order to ensure the system behavior.

The assessment and optimization for sizing the hybrid solar-wind power generation system were carried out taking into account important stages. First, the evaluation of the weather conditions in every case, its importance is because they are the raw material for the production of electrical energy by renewable systems. After that, the selection of mathematical models and the technologies to be used in each case, because the accuracy of individual component model and technology selected for each one express the precision of the entire system. An optimization method is then used to select the optimal configuration satisfying the user needs and restrictions, for that, a review of literature in hybrid solar-wind systems has shown that Genetic Algorithms own superior structures that make it common for optimization of hybrid renewable energy systems.

\subsection{Contributions}

This thesis makes the following contributions in hybrid renewable energy systems problems:

- An intelligent approach to dimensioning renewable energy systems able to adjust to the needs and limitations of different cases. For this, a computational system is presented capable of adjusting to the conditions of each case study.

- Improve the accuracy of the system reliability by having two approaches: annually and daily, in order to establish the system's minimum statistically reliable hours. 


\subsection{Reader's Guide to the Thesis}

Following is a general description of the contents of this dissertation. This master thesis is organized in three main parts distributed by chapters.

\section{Part I: Introduction and Related Works}

Chapter 1 presents a motivational introduction on the main topics, objectives and contributions regarding this dissertation.

Chapter 2 gives a general overview of background information regarding renewable energies: solar and wind systems, batteries, inverters, hybrid systems, intelligent optimization techniques and economic analysis criteria that are required to develop the propose approach described in chapter 4 and 5.

Chapter 3 provides a general survey of the most relevant work related to the research addressed in this thesis.

\section{Part II: Proposed Approach}

Chapter 4 describes the formal aspects of the hybrid renewable energy system presented in this thesis.

Chapter 5 presents the implementation of the approach proposed in chapter 4 . The chapter also contributes to complete the description of such proposal.

\section{Part III: Results and Conclusions}

Chapter 6 provides experimental results of the implemented approach. Several cases are presented to evaluate the performance of different criteria sizing hybrid systems with solar and wind energy in simulation runs, also a comparison with HOMER software is made.

Chapter 7 discusses and analyzes the results, summarizes the conclusions and contributions of the thesis and outlines the most promising directions for future work. 


\section{Chapter 2}

\section{Background Information}

This chapter introduces and reviews general concepts of renewable energies, hybrid systems, solar and wind energy, batteries, inverters and a briefly description of computational intelligent systems used for sizing hybrid systems, also the economic analysis techniques required for developing the proposed approach.

\subsection{Renewable energy}

Renewable energy can be defined as "Energy obtained from the natural and persistent flows of energy that occur in the immediate environment". An example of that is solar energy, which is repetitive over a 24-hour period. The source that creates renewable energy is the sun that is converted into different forms e.g. solar radiation to the energy of wind or waves. Such energy can also be called Green Energy or Sustainable Energy (Twidell \& Weir, 2006).

Figure 1 shows the energy flows that constantly pass as renewable energy across the Earth. However, the global data of Figure 1 has little value for engineering applications, as certain sites may have environments and extraordinarily different possibilities to take advantage of renewable energies. For instance, plane regions as Denmark have high opportunities for wind power but less for hydropower, Norwegian regions dominate with hydrological sources and countries like Colombia has more heterogeneous supply mix, as wind can dominate the North but hydropower dominates the central and south regions. Therefore, practical renewable energy systems must be adapted to the local environmental energy flows that occur in a given region. 
Alternative energy resources such as solar, wind, biomass, hydroelectric and geothermal, etc., have being used largely to generate energy in recent years. Solar and wind power systems are considered as promising sources of power generation due to their availability and topological advantages for local power generation in remote areas. The use of solar and wind energy has become increasingly attractive and profitable in every oil crisis.

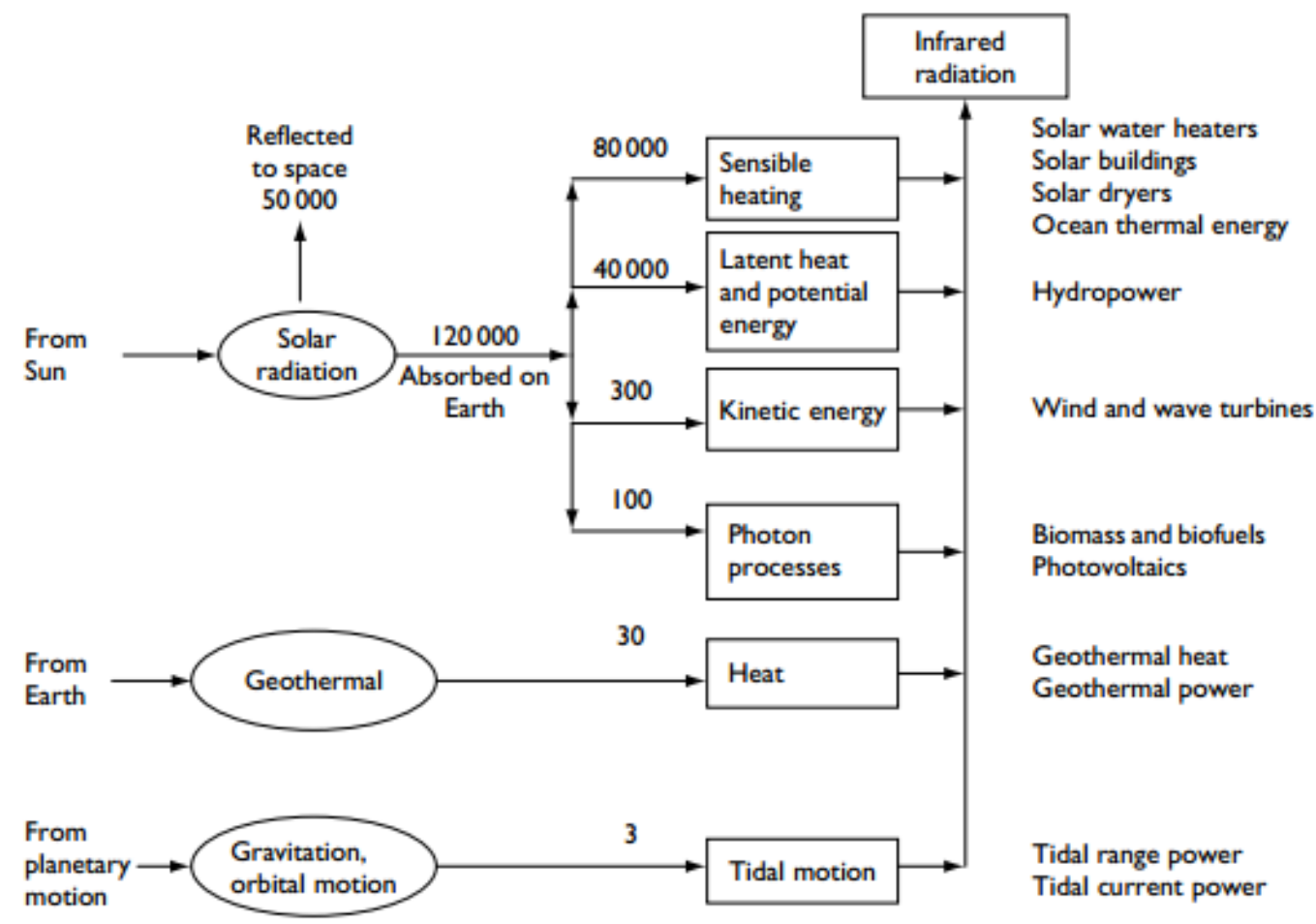

Figure 1. Natural energy currents on earth. Taken from (Twidell \& Weir, 2006)

\subsubsection{Solar Photovoltaic Energy}

Currently, there are only two methods of generating energy; the first one requires the use of an external motor or turbine and the second one is photovoltaic (PV) generation using solar cells. The PV effect was discovered by Becquerel in 1839, but was only developed as an energy source until 1954 by Chapin, Fuller and Pearson (Twidell \& Weir, 2006).

Solar cells are solid-state devices without moving parts that convert sunlight energy directly into DC electricity. They are made of two or more thin layers of semiconductor materials, usually silicon, and are specially treated to form an 
electric field with positive on one side (back) and negative on the other side (front side facing the sun). When the solar cell is exposed to light, the struck electrons are released from the atoms in the semiconductor material, creating electron-hole pairs. If the electric conductors are attached to the positive and negative sides, forming an electrical circuit, the electrons are captured as an electric current (Kalogirou, 2013).

The choice of cell material can have important effects on the design and performance of the system. Both the composition of the material and its atomic structure are influential. PV materials include silicon, gallium arsenide and many others. The atomic structure of a PV cell can be a single crystal, polycrystalline or amorphous. The most commonly produced photovoltaic material is crystalline silicon, either monocrystalline or polycrystalline (Kalogirou, 2013).

A PV generator is mainly a set of solar cells, connections, protective parts and supports. PV panels is the main element of a PV system and offer additional advantages over other renewable energy sources, as they emit no noise and require negligible maintenance; they also generate electricity without producing greenhouse emissions or any other gas. PV systems can be installed in a wider variety of locations (e.g. roofs and parking ceilings); a utility-scale power plant can be built in months, and is often located close to electricity users, which reduces transmission costs, these factors make PV electricity more "valuable" than a traditional power plant. PV systems can be built in virtually any size, from milliwatt to megawatt, and the systems are modular, that is, more panels can be easily added to increase output, as shown in Figure 2. It is also possible to configure them as stand-alone systems. 

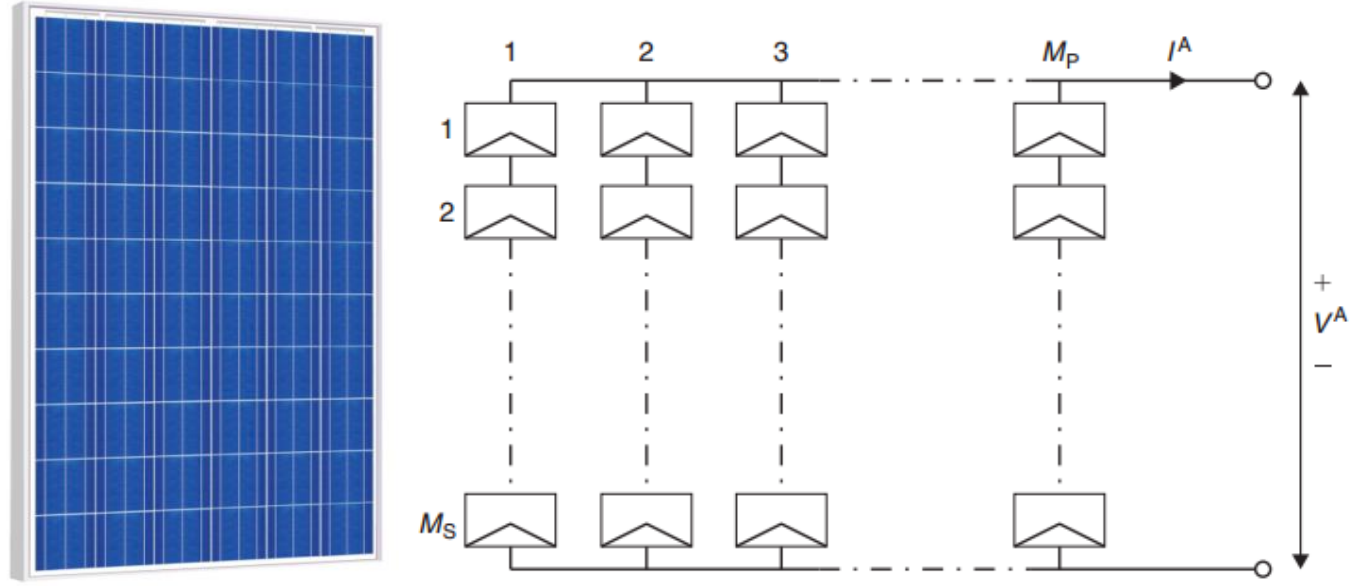

Figure 2. Cell array composition with any virtually size. Taken by (Twidell \& Weir, 2006)

\subsubsection{Wind Energy}

The winds are movements of masses of air in the atmosphere and an indirect action of the solar radiation that falls on the earth as they are generated mainly by differences of temperature. Therefore, wind energy can be considered a form of solar energy (Sørensen, 2004).

The device used to extract energy from the wind is the wind turbine (WT). They are considered a mature technology and it is an established industry. Nowadays, the machines are manufactured in a wide range of capacities that vary from $400 \mathrm{~W}$ to 7.5 MW and diameters from approximately $1 \mathrm{~m}$ to over $100 \mathrm{~m}$. The maximum rated power rating of a WT is given for a specified nominal wind speed, commonly around $12 \mathrm{~m} / \mathrm{s}$ (Twidell \& Weir, 2006). The maximum theoretical aerodynamic conversion efficiency of WTs from wind to mechanical power is 59\%. However, even the most efficient fundamental properties used for large and medium WT blades limit the maximum efficiency achievable to around 48\%. In practice, the need to economize on blade costs tends to lead to the construction of low-power WT, with optimum performance of $45 \%$ (Kalogirou, 2013).

The names of the different types of WT depend on their geometry of construction, and the aerodynamics of the wind that goes around the blades. The two main 
concepts of WTs are horizontal axis wind turbines (HAWTs) and vertical axis wind turbines (VAWTs). However, the world market is dominated by HAWT. Several designs have been proposed in the past in an attempt to reduce costs and increase efficiency; the main types are shown in Figure 3. Today, the HAWT basic system begins with a large rotor comprising a number of blades mounted on a horizontal axis at the top of a tower evenly distributed about the shaft, each blade having a suitable aerodynamic profile normally designed to produce a lifting force. Today most of the WT are manufactured with three blades, which is a compromise between the various types as they offer a smoother rotating operation, less noise and not a very high cost (Kalogirou, 2013).

Network-connected turbines and wind farms distribute energy to integrate with other forms of generation. For small applications connected to the grid, the generated AC electricity can be rectified to DC and then inverted to the AC grid frequency. For standalone applications, frequency and voltage do not need to be as standardized as end-use requirements vary. In all applications, it will be necessary to carefully adapt the characteristics of the machine to the local wind regime. In particular, sites with an average wind speed of less than $5 \mathrm{~m} / \mathrm{s}$ often have unacceptably long periods in which generation would not occur; however, if the annual average wind speed at $10 \mathrm{~m}$ in height is $5 \mathrm{~m} / \mathrm{s}$ or more, electricity generation can be contemplated (Sørensen, 2004).
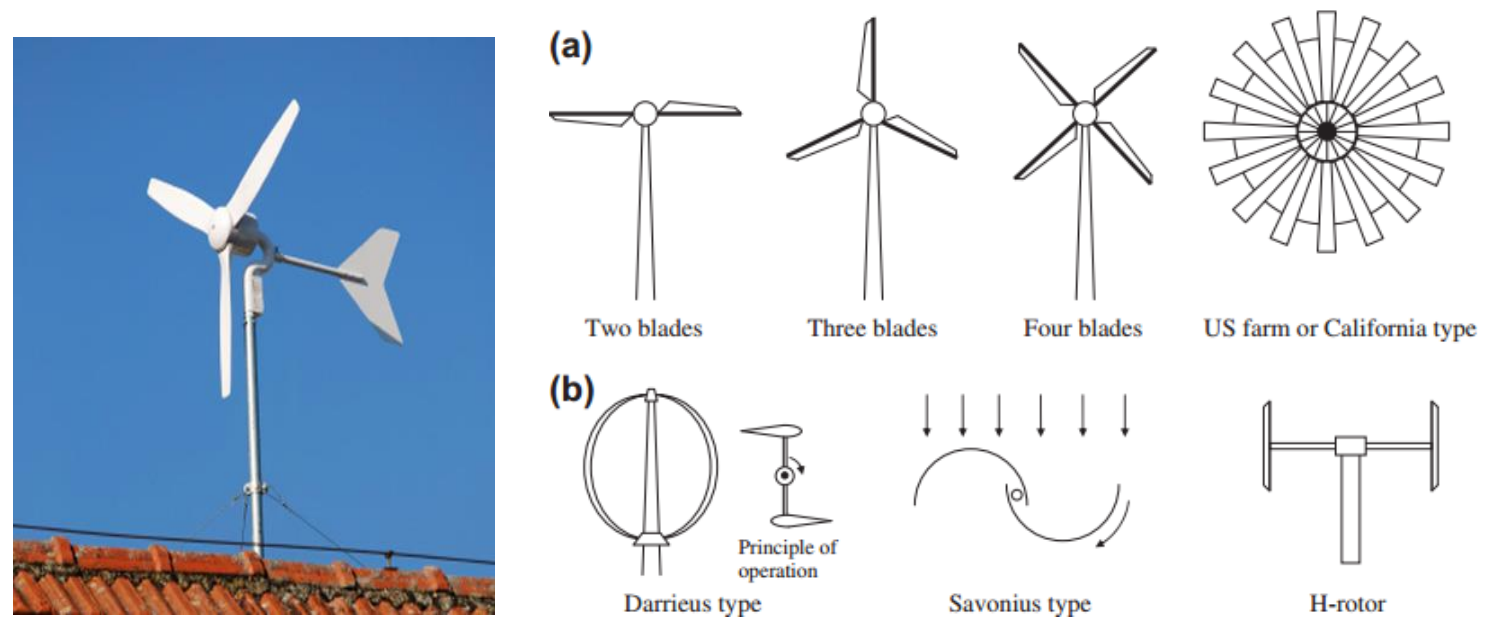

(b)
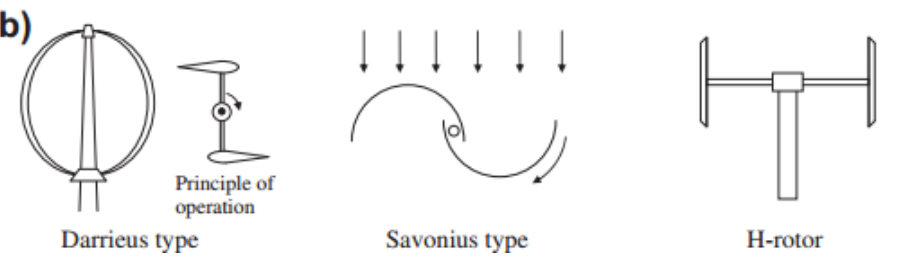

Figure 3. Main types of wind turbines. A) HAWTs, B) VAWTs. Taken by (Kalogirou, 2013) 


\subsubsection{Batteries}

Energy storage provides a way of adjusting to variations in energy demand, that is, a way of satisfying a load without time dependence. For renewable energy sources of a fluctuating nature, storage can help to make energy systems even as reliable as conventional systems. Generally, the low intensity and generalized location of most renewable sources favor decentralized end-use and the temporary dependency variable favors the integration of hybrid systems with storage to meet demand in real time; however, renewable energy supplies have different requirements for storage, including quick access and versatility of the way energy is delivered (Twidell \& Weir, 2006).

A device having both input and output electricity is called an electric accumulator. An electrochemical storage battery has properties determined by voltage, current, and time constants. Inside the battery, the ions are transported between the negative and positive electrodes through an electrolyte; this is why the electrodes can be solid, liquid or, in theory, gaseous (Sørensen, 2004).

The main types of batteries available today include lead acid, nickel cadmium, nickel hydride and lithium. Deep-cycle lead-acid batteries are the most commonly used, but despite considerable development efforts aimed at the power sector, battery storage is still restricted in practice to small-scale. The selection of the type and size of the battery depends mainly on the requirements of load and availability. When using batteries, they should be located in an area without extreme temperatures and the space where the batteries are located must be adequately ventilated (Sørensen, 2004).

Batteries are rated by their nominal capacity (qmax), which is the number of ampere-hours (Ah) that can be fully extracted from the battery under predetermined discharge conditions. The efficiency of a battery is the ratio of the extracted load (Ah) during discharge divided by the amount of load (Ah) needed to restore the initial state of charge (SOC). Therefore, the efficiency depends on the SOC 
and the load and discharge current. The SOC is the ratio of the current capacity of the battery to the rated capacity (Rangnekar, et al. 2016).

The primary requirement of batteries for a photovoltaic system is that they must be able to accept repeated loads and deep discharges without damage. Although photovoltaic batteries look similar to car batteries, the latter are not designed for repeated deep discharge and should not be used. For more capacity, the batteries can be arranged in parallel as shown in Figure 4.

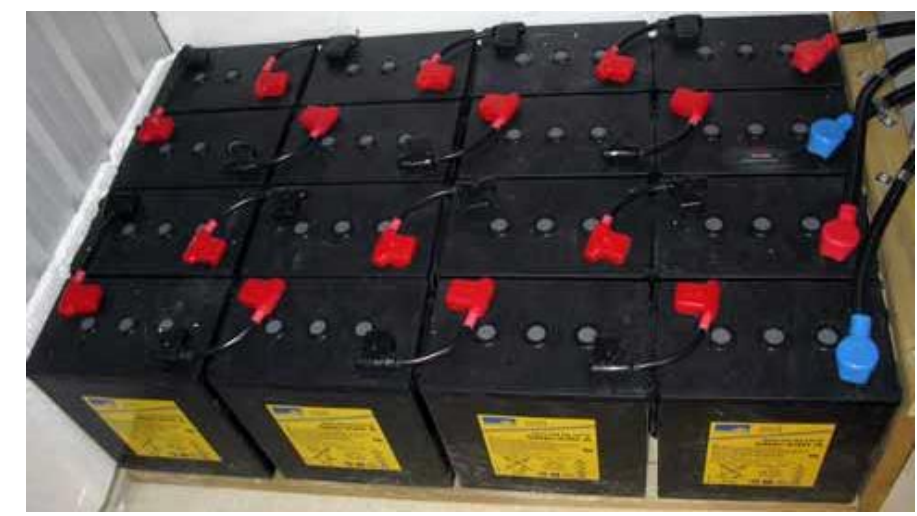

Figure 4. Battery system connection with 16 batteries.

\subsubsection{Inverters}

The inverters are the devices that convert DC energy into AC energy. These are necessary because most of the devices have AC connection like the grid; however, solar panels and batteries run on DC power. For a renewable installation with alternative energies, the inverter must be sized so that it can cope with the overload currents associated with starting motors, but not so large that it does not have a good cost-benefit ratio and work with little efficiency. Many inverter manufacturers offer the possibility of an overcurrent in the inverter for a specific time (Normally 530 minutes) (Kalogirou, 2013).

The waveform of the $\mathrm{AC}$ wave can be a square wave (cheap inverter) or an almost pure sine wave (solid-state electronic inverter). Solid-state electronic inverters are commercially available with excellent reliability and efficiency ( $\sim 95 \%)$. There are in the market investors that can vary in size, functionality and applications 
according to the needs of the system (Twidell \& Weir, 2006). They can be connected in parallel in order to increase the power capability as shown in Figure 5. There are several types of inverters, these depend specifically on the use that is required, among these are:

Grid-Tie Inverter (GTI): These inverters have the ability to connect the energy generated by the renewable system to the grid; for this, they synchronize the phase, the voltage (A little higher than the network so that there is flow out of the grid) and the frequency with the grid. Its main disadvantage is that if the grid power is not available, the inverter does not fulfill its function and therefore the end user has no service (Jana, et al., 2016).

Grid-Off Inverter: The inverters outside the network do not have to match the sinusoidal phase of the network. A stand-alone inverter uses an internal frequency generator and switching circuitry to transform the low voltage DC power to higher voltage AC power.

Hybrid inverters: Hybrid inverters operate mainly as Grid-tie inverters, however, they have the possibility to operate without being connected to the grid and in addition, they can manage the battery system in an efficient way.

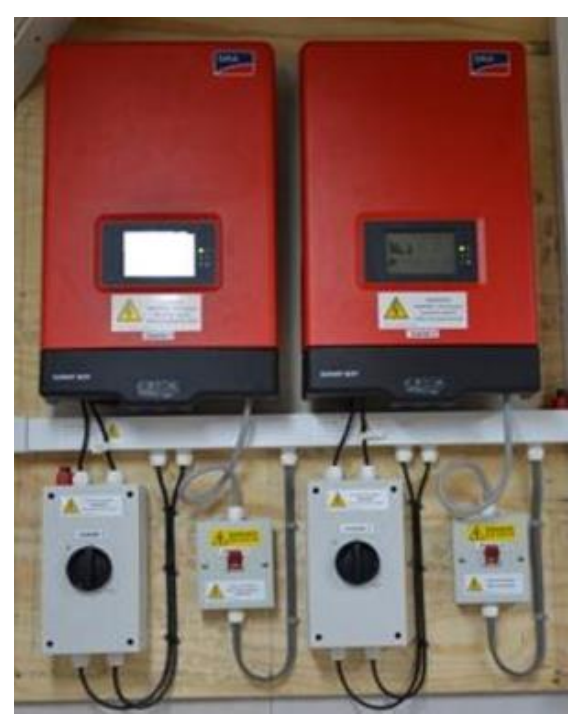

Figure 5. Connection of two inverters in parallel for hybrid systems 


\subsection{Hybrid system}

Hybrid systems are a competent choice to exploit power generation sources. The main characteristic of hybrid systems is that it consists of several sources of energy generation, which at least one of them being a renewable energy source. This is done in order to use the advantages of one type of energy used to complement the weaknesses of the others, to overcome the dependence of a specific type of source and to avoid the oversizing of components. In addition, these types of systems increase reliability and energy security, and reduce the cost of maintenance and emission of pollutants that are a global problem (Rangnekar et al., 2016).

The most commonly used sources for hybrid energy systems are wind, solar, biomass, geothermal, diesel and gasoline generators. This type of systems can be grid-connected or autonomous, and if adds energy storage it can overcome the reliability problems given by the intermittent resources. Solar photovoltaic power is considered the most popular and widely source used today. As for small loads, the most used hybrid systems are the photovoltaic and wind solar combination; this is due to the complementarity of wind speed-solar radiation resources (Twidell \& Weir, 2006).

The type of system installed and its configuration depend mainly on the availability of the resources to be implemented. Other aspects to be considered in the design and installation of a hybrid energy system is the load demand profile, economic constraints and local regulations for its implementation. With that, it is necessary to perform an optimization to make decisions and make optimal designs.

\subsection{Intelligent Optimization Techniques}

Renewable systems for energy generation such as solar and wind power are dependent on the resources of solar radiation and wind speed which are variable in each zone. A suitable design of a hybrid power generation system seeks, at least, to maximize power at low costs. Therefore, there is an increasing interest in the use of 
new generation optimizers, which are called artificial intelligence techniques. Intelligent optimization refers to an extended area of research that includes the use of memory, adaptation, incremental model development, experimental algorithms, intelligent fit, and heuristic design (S.N. Sivanandam \& S.N. Deepa, 2008), (Al Busaidi, et al., 2016).

There are different artificial intelligence based/metaheuristic/heuristic/nature inspired techniques that are widely used to optimize a hybrid energy system; Among these are "GA Genetic Algorithm", "PSO Particle Swarm Optimization”, "Tabu Search", "NN Neural Network", “ SA Simulated Annealing” (Sinha \& Chandel, 2015), (Mahesh \& Sandhu, 2015), (Zhou et al., 2010), (Al Busaidi, et al., 2016).

Genetic Algorithm is an adaptive heuristic search algorithm that simulate the process of natural and genetic selection developed by John Holland. Its operation is based on generating diverse solutions using techniques like mutation, selection and crossover. GA are applicable in various areas for problem solving and this is due to the advantages it has, like the ability of solving problems with multiple solutions, it is easy to understand and also always considers a population of solutions which makes it interesting, in addition to their adaptability. However, it has some limitations as a tendency to converge towards local optimum or even arbitrary points, it is a fairly general algorithm so that for specific problems other algorithms can be used that assure more rapidity and accuracy in the response, also it can not assure an exact time for the optimization response (S.N. Sivanandam \& S.N. Deepa, 2008).

The general operation of the GA follows these steps:

1. An initial population of solutions is randomly generated and represented as chromosomes in the form of genes. In that, each chromosome represent a possible solution of the problem and the genes are the workspace.

2. The objective function is used to evaluate the chromosomes and with that, each chromosome is ranked in terms of its fitness. 
3. A new generation is created depending on the ranking of the last population. Candidate solutions are selected based on their performance, the remaining set is generated by mutation and crossover functions.

4. The process of selection, mutation and crossover is repeated until one stop criteria is reached, in most cases, until the solution converges to a stationary value.

Particle swarm optimization, developed by Kennedy and Ebarjart, is a stock-based iterative stochastic optimization practice, guided by the social behavior of birds and fish. It has advantages such as convergence in a short time, easy to implement and complete. However, it has limitations as it can fall into local optimums and it cannot solve problems of the non-coordinated system.

Tabu Search, initially proposed by Glover, is an iterative heuristic algorithm that starts from a random initial solution and seeks to explore the space to find a better solution that escapes local minimums, avoiding solutions already found. Some cases, it evolves from a good solution to a worse looking after that find an even better solution than the initial one (Pirim, et al. 2008).

Neural Network is a group of connected artificial neurons trained based on observed data that uses a mathematical or computational model for information processing based on a connectionist approach to computation in order to solve problems by modeling the human brain information processing. The main advantage of Neural Network is the learning process and the architecture, also its applicability in different situations (F. Bilal, 2013).

Simulated Annealing, developed by Kirkpatrick, Gelatt and Vecchi was inspired by natural phenomena. It is a trajectory based on the random search technique for global optimization, and is based on the annealing process. This technique is recognized by it robustness and versatility dealing with highly nonlinear models, chaotic and noisy data and many restrictions. The main advantage of SA is its ability 
to avoid being trapped in local minimums; however, the main weakness of SA is that the quality of the result may be poor (Katsigiannis, et al. 2012) (danial kitchener, n.d.).

\subsection{Economic Analysis}

Inflation is an economic phenomenon that consists in the loss of the purchasing power of money over time, that is, the value of money changes over time. This makes forecasting future values more complex than just adding and subtracting (Baca Urbina, 2007). To calculate the present value (P) of money to be invested in the future (F) after $n$ periods, with an interest rate $i$, equation (1) is used:

$$
P=\frac{F}{(1+i)^{n}}
$$

This equation is called the equivalence formula of money over time. However, when making an investment, the scheme that is usually proposed is: Is it convenient to make the investment? In order to solve these questions, the concept of Net Present Value NPV arises which indicates if the investor is gaining an approximate percentage of profit; for this, investors must transfer the flows of the future years to the present time and subtract the initial investment that is already in present time. In the context of this research, it is important to know if it is profitable or not to install a hybrid system designed in order to know in each case the years of return on investment and annual profits. 


\section{Chapter 3}

\section{Related Work}

This chapter presents an overview of the main works focused on the topics addressed in this dissertation.

\subsection{Review of hybrid solar-wind systems sizing}

Several studies have been carried out around hybrid systems with solar and wind energy. For grid-connected systems, (Subrahmanyam, et al., 2012) worked on a system able to supply electricity to a private house, farmhouse or small company without optimize the system components. (Bayod-Rújula, et al. 2013) analyzed the influence of the parameters of a grid connected hybrid wind-PV system with energy storage; with that, they proved the importance of hybrid systems and the reliability and cost reductions in comparison with non-hybrid systems; the importance of a proper sizing is that the over-sizing increases the investment money and the electrical looses in the line. (Caballero, et al., 2013) developed a method for optimal sizing a HRES with the optimization approach of minimize the Life Cycle Cost (LCC) of the system; this method, allows to sell energy to the grid and receive it in a Net Metering Scheme. (Alsayed, et al., 2013) developed an optimal sizing system taking into account different criteria: environmental, economic and social performance; in order to achieve the optimal sizing of the system with different criteria, they used MCDA algorithm to solve it. (Arabali, et al., 2014) proposed a stochastic method to optimally size a hybrid system; in that, ARMA is used to model wind speed, solar radiation and load; Montecarlo simulation is used to make the mono-objective optimization of reliability requirements. (González, et al., 2015) worked with GA to optimize the system, the NPV is the mono-objective function of the optimization and the parameters are area occupied by solar panels and number of wind 
turbines.(Sinha \& Chandel, 2015c) and (Khare, et al., 2016) made a review of hybrid solar systems grid-connected and off-grid; they found that different optimization techniques have been used and all of them give different accuracy and execution time. The most used approaches optimization are divided in to groups: Reliability criteria and cost analysis method.

For off-grid hybrid solar-wind systems, (Belmili, et al., 2013) developed a software able to size a system under certain conditions; the optimization is made with the Loss of Power Supply Probability approach; the weather conditions data is taken from the NASA with the user given the latitude and longitude data of the zone, also the user must give the technical parameters of elements. (Ma, Yang, \& Lu, 2014) presented a feasibility study of and techno-economic evaluation for a remote island. The simulations were done by using HOMER software, the optimization approach was the Levelized Cost of Energy COE. (Grouz \& Sbita, 2014) designed a system for an on sea oil wellhead electrification this study seeks to minimize the total cost of the system. (Mokheimer, et al., 2015) developed a computational model for sizing optimization using Matlab tool; the optimization approach is Loss of Power Supply Probability LPSP. (Al Busaidi, et al., 2016) made a review of the techniques used to optimize hybrid systems; the optimization techniques are divided into approaches, the conventional techniques and the artificial intelligent techniques; in this study is recommended the use of hybrid systems due to the increase of reliability and decrease of Initial Capital Cost ICC and Life Cycle Cost LCC. (Al., 2017) proposed a new method for optimal sizing a hybrid system with a statistical approach that aims to exploit the capacity of a hybrid supercapacitor-battery storage system; a frequency management and a hysteresis controller have been also suggested to control the output of the storage units. The proposed study also purposes to smoot the variations in wind-solar power production and ensure a more controllable output power.

For both, off grid and grid connected systems there are also several studies. (Xu, et al., 2013) developed an optimal system for grid-connected and off-grid mode, looking for achieving high power supply reliability, making full use of the 
complementary characteristics of wind and solar, ensuring a small fluctuation of power injected into the grid, optimizing the batteries State Of Charge SOC and minimizing the total cost of the system. The optimization is made with an improved method and the mono-objective optimization approach is minimizing the system cost. (Saharia, et al., 2016) worked on a renewable energy system for electrification of an education institution; the system was proved for stand-alone and gridconnected system and optimized by HOMER; they showed that the cost of energy is high in stand-alone mode.

\subsection{Intelligent optimization techniques}

In this research, we carried out a study with the available literature to explore the most intelligent optimization techniques used to the optimal sizing hybrid renewable energy systems with solar PV and wind energy generation systems. This was done in order to select the intelligent optimization technique to be used for the system to be implemented. Figure 6 shows the comparison of the techniques found.

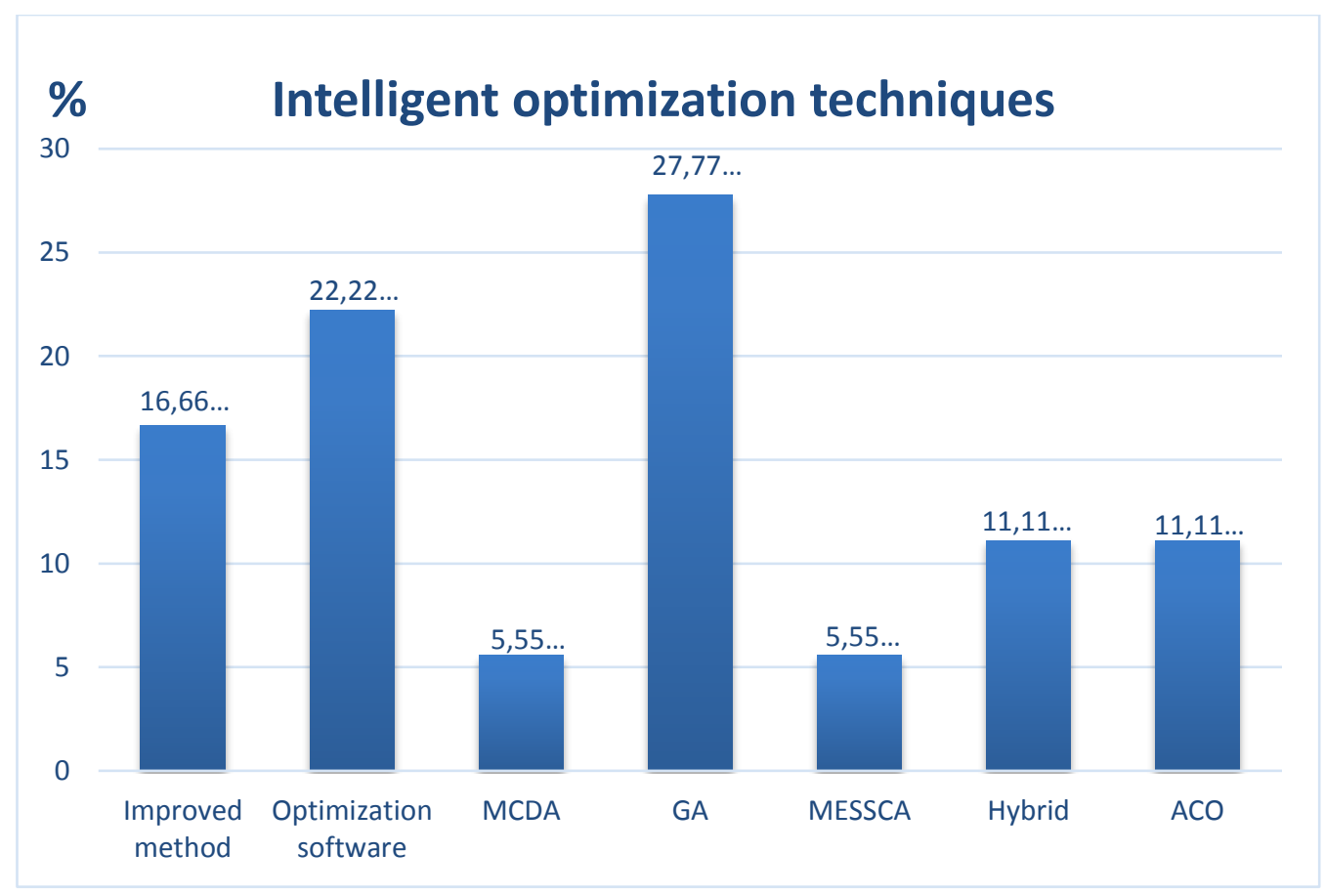

Figure 6. Comparison between intelligent optimization techniques used to optimize hybrid wind-solar systems. 
Figure 6 shows that the most common intelligent optimization technique used is GA, however the $22,22 \%$ of the studied papers prefer to use software like HOMER.

\subsection{Optimization approaches}

In this research, we carried out a study to explore the most used optimization approaches to optimally size hybrid renewable energy systems with solar PV and wind energy generation sources. Figure 7 shows the comparison of the optimization approaches found. For that,

Initial Capital Cost (ICC): it refers to the initial cost of the installed system.

Life Cycle Cost (LCC): it refers to the total cost of the system during its cycle life.

Loss of Power Supply Probability (LPSP): It refers to the power that cannot be supplied from the total demand.

Cost of Energy (COE): it refers to the cost of electricity generation as the ratio of total annualized cost of the system to the annual electricity delivered by the system Annualized cost of system (ACS): is defined as the ratio that includes all the costs over the system's lifetime to the years of the system life cycle cost.

Net Present Value (NPV): is defined as the ratio of the total annualized cost of the system to the annual electricity delivered by the system.

Loss of Power Supply (LPS): it refers to the hour of the day that the power is not $100 \%$ supplied.

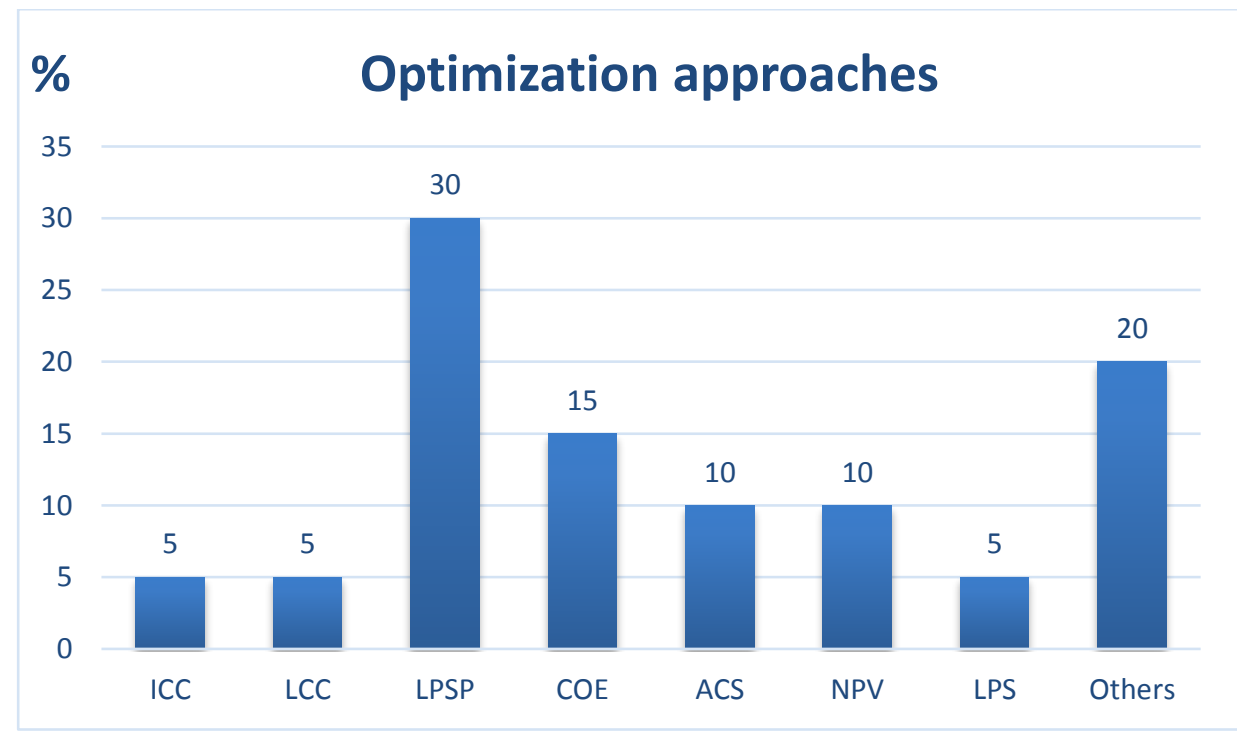

Figure 7. Comparison between optimization approaches used to optimize hybrid systems. 
Figure 7 shows that there is a great variety of optimization approaches used; however, the most commons are in reliability LPSP and LPP and in cost ACS and COE.

\subsection{Mathematical models}

The selection of the mathematical models of the main components of the system were made according to the most common and complete models used in literature. Results found are presented below.

These models need specific values from the physical element. For each case study, there will be three options for each subsystem of the hybrid system (solar, wind and batteries). The first one is that the system will select the reference element that best suits the design which means that it has a backup of specifications that will be constantly updated (For each element, the backup of selected references is shown in Appendix). Second, enter a specific reference with which the design will be performed, for that, some specifications of each elements will be required, and third select one of the options in the backup of the system.

\section{Solar Panel:}

A study was carried out to select the model of the solar panel. Twelve (12) models used for solar panels in published studies were analyzed. The main components of each model are shown in Table 1 . The most common and complete mathematical model founded was the selected one.

\section{Wind turbine:}

A study was carried out to select the model of the wind turbine. Twelve (12) models used for wind turbines in published studies were analyzed. The main components of each model are shown in Table 2. The most common and complete mathematical model founded was the selected one. 


\begin{tabular}{|c|c|c|c|c|c|c|c|c|c|}
\hline & 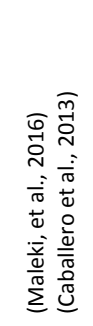 & 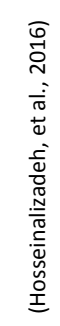 & 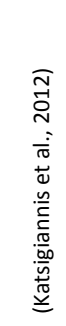 & 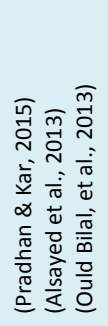 & 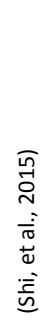 & 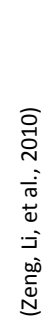 & 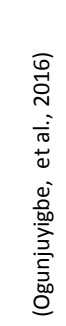 & 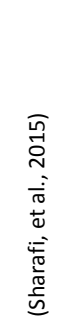 & 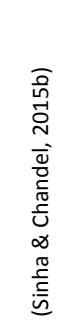 \\
\hline Solar radiation & $\checkmark$ & $\checkmark$ & $\checkmark$ & $\checkmark$ & $\checkmark$ & $\checkmark$ & $\checkmark$ & $\checkmark$ & $\checkmark$ \\
\hline $\begin{array}{l}\text { Surface area panel } \\
\text { number }\end{array}$ & $\checkmark$ & $\checkmark$ & & $\checkmark$ & $\checkmark$ & & $\checkmark$ & $\checkmark$ & $\checkmark$ \\
\hline Module temperature & & $\checkmark$ & $\checkmark$ & $\checkmark$ & $\checkmark$ & $\checkmark$ & & & $\checkmark$ \\
\hline Ambient temperature & & $\checkmark$ & $\checkmark$ & $\checkmark$ & $\checkmark$ & $\checkmark$ & & & $\checkmark$ \\
\hline Module efficiency & $\checkmark$ & $\checkmark$ & & & & & $\checkmark$ & $\checkmark$ & $\checkmark$ \\
\hline Fill Factor & & & & $\checkmark$ & $\checkmark$ & & & & \\
\hline Derating factor & & & $\checkmark$ & & & & & & \\
\hline Vco(stc) & & & & $\checkmark$ & $\checkmark$ & & & & \\
\hline Isc(stc) & & & & $\checkmark$ & $\checkmark$ & & & & \\
\hline NOCT & & $\checkmark$ & $\checkmark$ & $\checkmark$ & $\checkmark$ & & & & \\
\hline Nominal power & & & $\checkmark$ & & & $\checkmark$ & $\checkmark$ & & \\
\hline Others & & & & & $\checkmark$ & & $\checkmark$ & $\checkmark$ & \\
\hline
\end{tabular}

Table 1. Studied mathematical model for solar panel.

\begin{tabular}{|c|c|c|c|c|c|c|c|c|}
\hline & 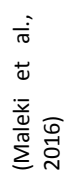 & 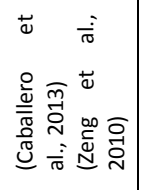 & 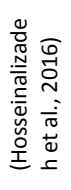 & 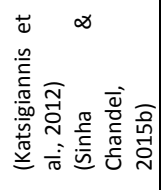 & 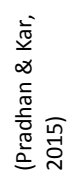 & 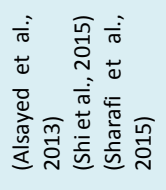 & 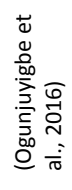 & 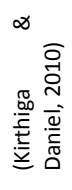 \\
\hline Wind speed & $\checkmark$ & $\checkmark$ & $\checkmark$ & $\checkmark$ & $\checkmark$ & $\checkmark$ & $\checkmark$ & $\checkmark$ \\
\hline Power Coefficient & $\checkmark$ & & $\checkmark$ & & $\checkmark$ & $\checkmark$ & & $\checkmark$ \\
\hline Vci (Cut in speed) & $\checkmark$ & $\checkmark$ & & $\checkmark$ & & $\checkmark$ & $\checkmark$ & \\
\hline $\operatorname{Vr}$ (rated speed) & $\checkmark$ & $\checkmark$ & & $\checkmark$ & & $\checkmark$ & $\checkmark$ & \\
\hline Vco (cut off speed) & $\checkmark$ & $\checkmark$ & & $\checkmark$ & & $\checkmark$ & $\checkmark$ & \\
\hline Generator rated power & $\checkmark$ & $\checkmark$ & & & & $\checkmark$ & $\checkmark$ & \\
\hline Area swept & $\checkmark$ & & $\checkmark$ & & $\checkmark$ & $\checkmark$ & $\checkmark$ & $\checkmark$ \\
\hline High relation & & & $\checkmark$ & & & $\checkmark$ & & $\checkmark$ \\
\hline Efficiency gear box & $\checkmark$ & & & & $\checkmark$ & $\checkmark$ & & \\
\hline Turbine efficiency & $\checkmark$ & & & & $\checkmark$ & $\checkmark$ & $\checkmark$ & \\
\hline Others & & & & $\checkmark$ & $\checkmark$ & & & \\
\hline
\end{tabular}

Table 2. Studied mathematical models for wind turbines.

\section{State of charge of battery (SOC):}

A study was carried out to select the model of the batteries. Seven (7) models used for batteries in published studies were analyzed. The main components of each model are shown in Table 3. The selected model was the most used in the studies 
analyzed; in addition, it was taken into account the required parameters because some of them are not available in batteries datasheets.

The SOC of the battery depends on the previous SOC and the possible situations that may arise: 1. Charging state of batteries: the power-generated system exceeds the load demand. 2. Discharging state of batteries: the load demand exceeds the powergenerated system. The efficiency of the batteries at discharging state is assumed depreciable.

\begin{tabular}{|c|c|c|c|c|c|}
\hline & 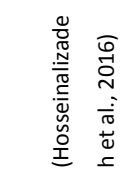 & 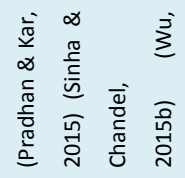 & 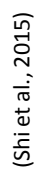 & 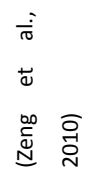 & 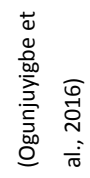 \\
\hline SOC before & & $\checkmark$ & $\checkmark$ & $\checkmark$ & \\
\hline $\begin{array}{l}\text { SOC } \\
\text { minimum }\end{array}$ & & $\checkmark$ & $\checkmark$ & $\checkmark$ & \\
\hline $\begin{array}{l}\text { SOC } \\
\text { maximum }\end{array}$ & & $\checkmark$ & $\checkmark$ & $\checkmark$ & \\
\hline Efficiency & & $\checkmark$ & $\checkmark$ & & $\checkmark$ \\
\hline$\sigma$ & & $\checkmark$ & & & \\
\hline Power & $\checkmark$ & $\checkmark$ & $\checkmark$ & & \\
\hline Voltage & $\checkmark$ & & $\checkmark$ & $\checkmark$ & $\checkmark$ \\
\hline Current & & & & $\checkmark$ & \\
\hline $\begin{array}{l}\text { Depth of } \\
\text { discharge }\end{array}$ & $\checkmark$ & $\checkmark$ & & & $\checkmark$ \\
\hline Others & & & & & $\checkmark$ \\
\hline
\end{tabular}

Table 3. Studied mathematical models for batteries SOC.

\subsection{Final Remarks}

Several studies have been carried out in order to optimally size hybrid renewable energy systems with solar and wind energies. In literature, there is a great variety of designs that apply to optimum sizing these systems, as well as different ways of analyzing climatic conditions, mathematical models developed for each technology used, optimization approaches asnd intelligent techniques. The design characteristics are in most cases established by the researchers for specific conditions but do not allow great variability of the system inputs. In addition, only one work was found which optimized also different possible technologies and its 
important because it influences considerably in the cost and performance of the system.

In the later chapters, the development of a system that allows for different cases with different restrictions and configurations optimally size the solar-wind hybrid system is shown. Within the allowed configurations, there are grid connected and off-grid systems, in addition, each one of them has the option of having a storage system or not. A new method of analyzing reliability with weather conditions is implemented which allows having two different kinds of reliability (annually and daily). The reference of the elements can be optimized. The system presented is not subject to specific possibilities because it is adaptable and flexible according to the needs of each particular case where it is desired to be implemented. 


\section{PART II}

PROPOSED

APPROACH 


\section{Chapter 4}

\section{Hybrid Renewable Energy Systems}

\section{Approach}

This chapter presents the proposed approach in this dissertation applied to hybrid renewable energy systems. The main definitions, general considerations, algorithms for the statistical analysis of random weather variables and load, model of the components of a hybrid solar-wind system and optimization technique used in this thesis are introduced in this chapter.

\subsection{Problem Statement}

In order to contextualize the proposed system, Figure 8 shows this thesis context, which refers to the generation of electrical energy through renewable energy sources to optimally size systems with loads with less than $5 \mathrm{~kW} / \mathrm{h}$. For the correct use of renewable energy sources, hybrid systems (systems with two or more generation sources and at least one of them renewable) are used, with solar and wind resources due to their complementarity and their great use in hybrid renewable small-scale systems. This topic is widely researched today due to its multiple benefits, highlighting among them the ability to generate clean energy for systems off-grid and decrease in the bill energy and support to grid-connected systems.

There are different combinations of hybrid systems with renewable energy sources according to the needs of the user. However, dimensioning each of these supposes a different study for each case. In literature, it is possible to find different studies for 
specific cases of users with different types of limitations and restrictions; however, there are no studies that cover the optimization of hybrid solar and wind systems open to any case.

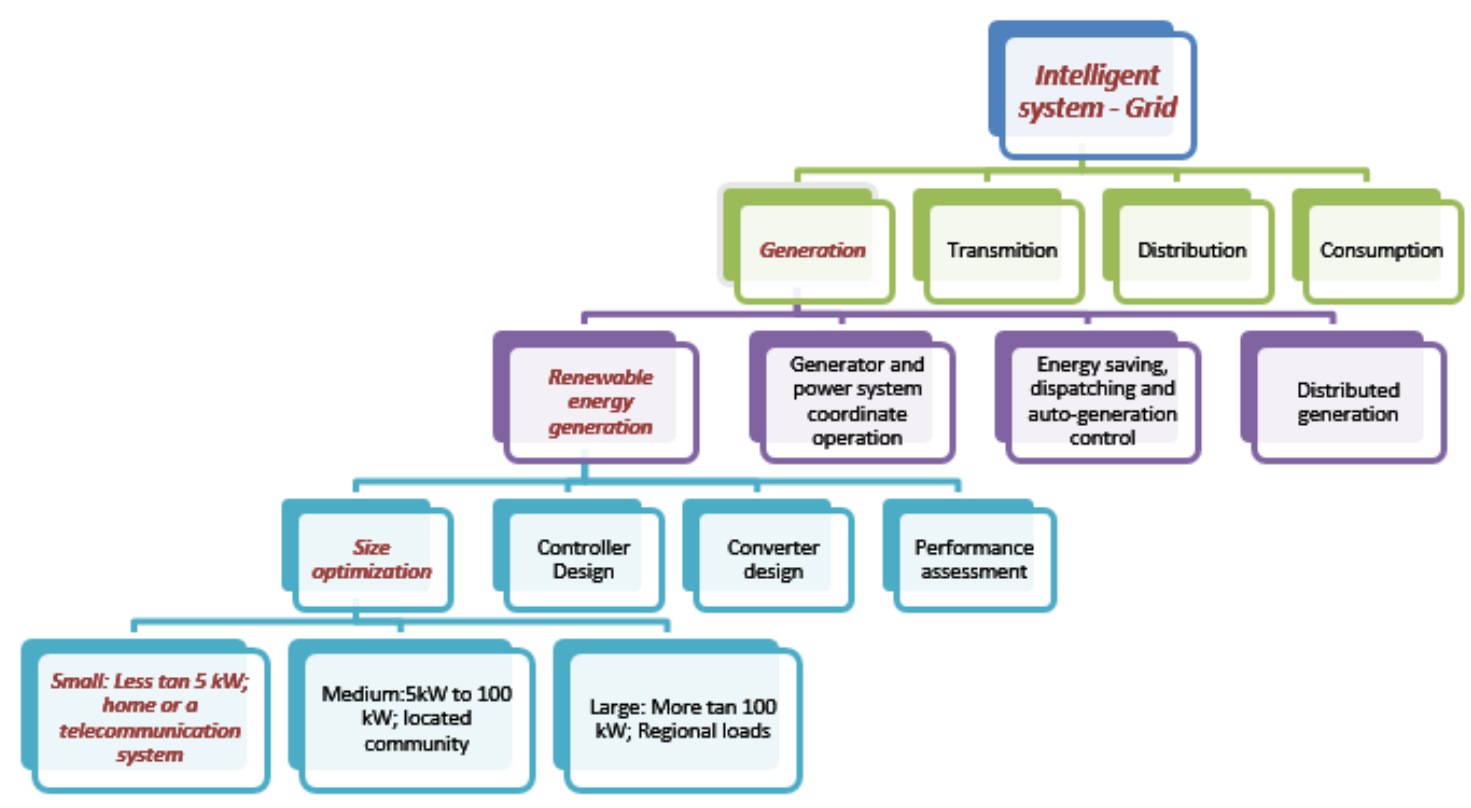

Figure 8. Thesis context

\subsection{Proposed Hybrid System}

The proposed hybrid system is shown in Figure 9. The main components of the system are solar panels and wind generators (renewable sources to generate electricity, the selection and the amount of them will depend of each case study, load and weather conditions), the battery bank (optional) in order to save electricity for low generation periods and an inverter to convert DC electricity into AC electricity for user needs and grid (optional).

The development of the optimum sizing system for hybrid power generation with solar and wind sources was performed as shown in Figure 10. 


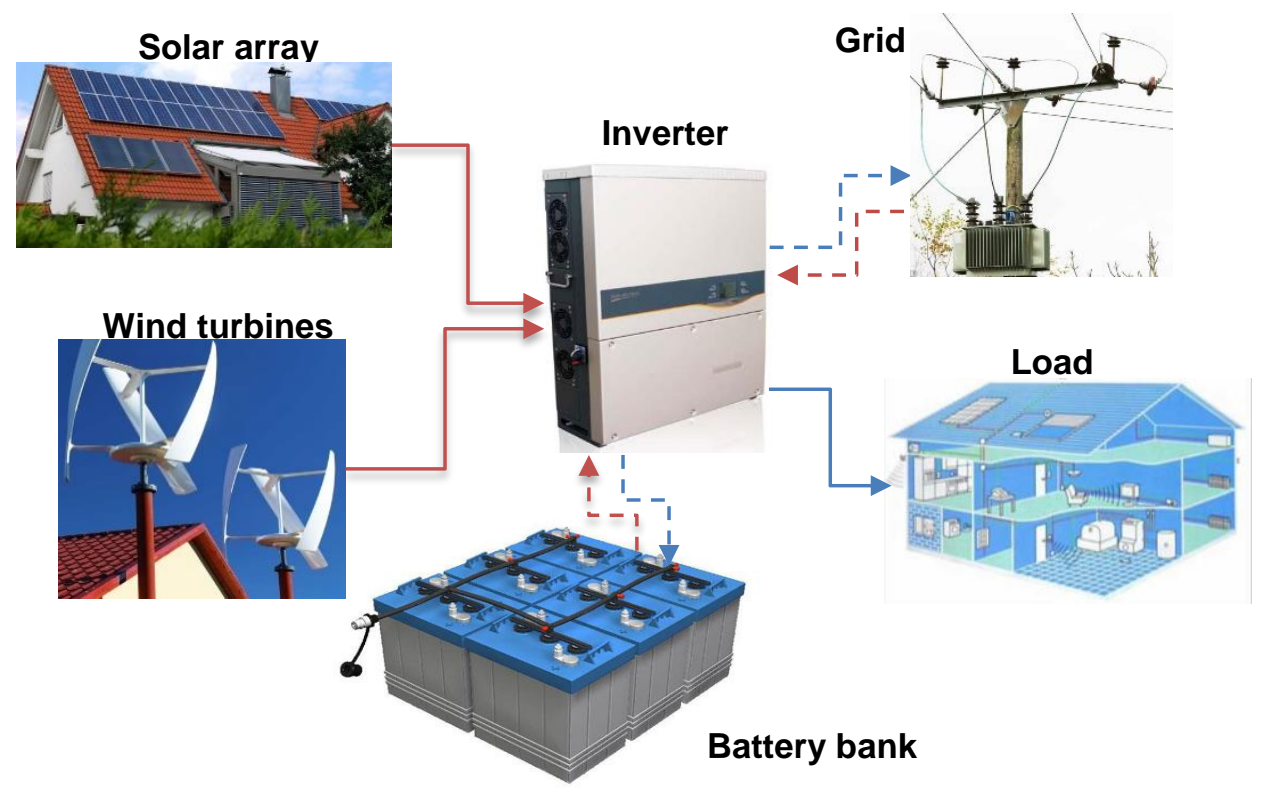

Figure 9. Schematic of the proposed hybrid renewable system

5.

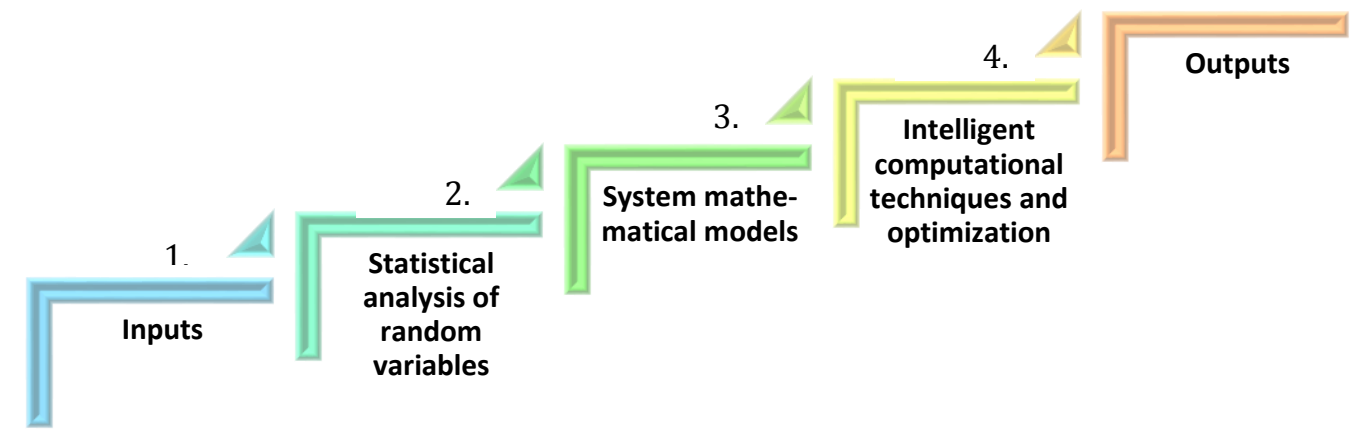

Figure 10. Steps for system implementation.

1. Definition of the system inputs. It seeks to create system able to be adapted to any configuration to meet the basic needs and requirements in each case.

2. Compilation and statistical study of meteorological data.

3. Development or selection of the mathematical models of the energy sources, batteries, inverter and load.

4. Selection, analysis, design and implementation of the optimization technique. The approaches in which the optimization of the hybrid system will be focused are also defined in this step, and finally

5. System results. 


\subsubsection{Inputs}

The Inputs are set looking to give freedom to the system to be adapted to any case study. It is possible to divide the system inputs in three large groups. This is presented in Figure 11.

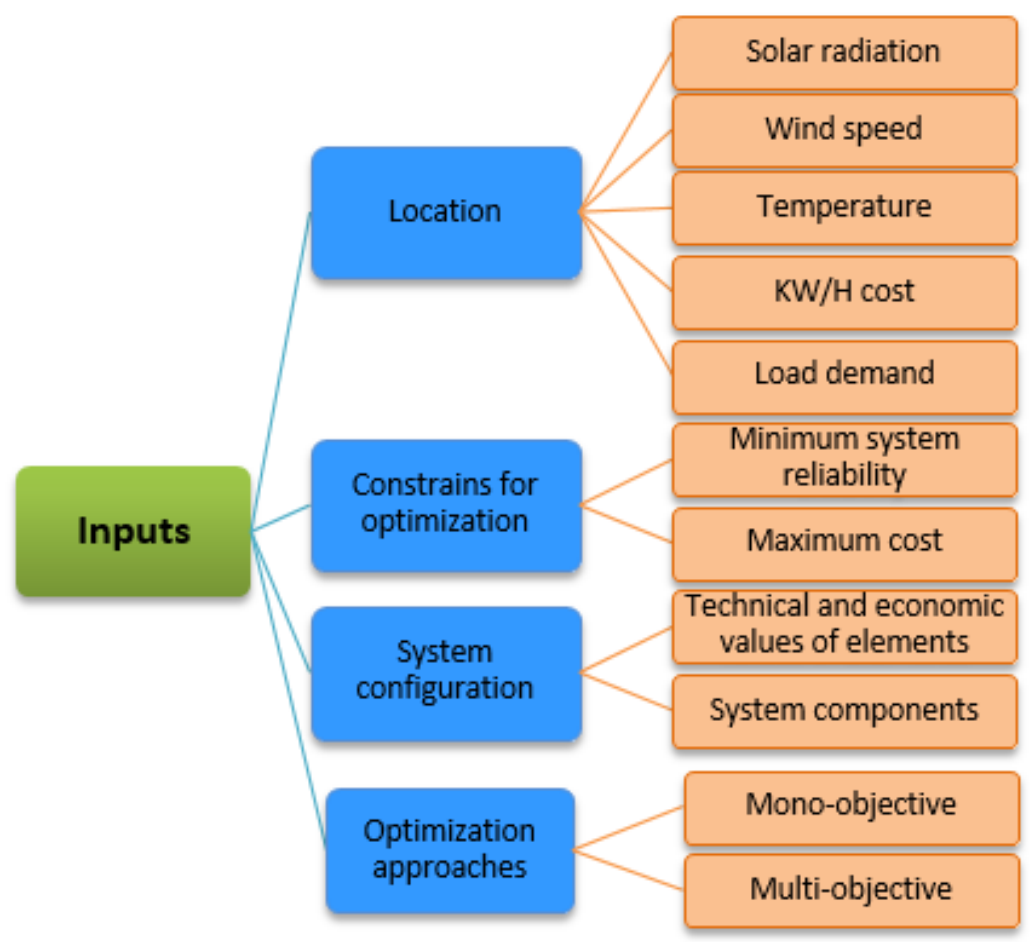

Figure 11. Inputs of the system

The first group is Location variables. This includes the random variables of the system as solar radiation, wind speed and temperature; as far as possible, it is desirable three-year history data in order to obtain better results due to the tendency and stochasticity of the data; however, at least one year history data could work. It also includes the $\mathrm{KW} / \mathrm{h}$ price in order to perform saving cost calculations and power purchase charges if the system grid-connected.

The second group is called constraints for optimization; in this stage, the values of minimum reliability and maximum cost are defined. In addition, available area for solar panels and the maximum number of wind turbines adjusted to each case study are required. 
System configuration inputs include the technical and economic values of the elements to be installed. Three options are available: The first is a backup of references of the elements of the system; for this, one of the references shown must be selected (references in the backup are presented in Appendix). The second one is the optimization of the reference to select the best among the backup. The last one refers to the fact of testing a specific reference in a certain case study, for this, the required values must be entered for each element.

In terms of configuration, for the development of this thesis, four possibilities were taken into account in order to cover the particular cases found in literature: Connected to grid with batteries, connected to grid with the possibility to sale the surplus energy to grid, grid-connected and off-grid. Finally, the capability of select one or two optimization approaches from a presented list.

\subsubsection{Statistical analysis of random variables}

The energy generation of systems with renewable energy sources depends on the climatic conditions of the area where the system is going to be implemented, because for each place the conditions change. Therefore, for optimum sizing of the hybrid systems, the analysis of the meteorological data is a very important step in order to dimension the amount of power generated.

The required meteorological data are mostly available at local weather stations. For this, after having defined the configuration of the system, it is necessary to obtain data of solar radiation, temperature, wind speed and load demand. Different methods can be found in literature to collect data; intervals from 1 year to 30 years to obtain a clear understanding of patterns, data generated with statistical methodologies (especially when there is no data is available) and the annual average monthly values are some of them.

In this work, a new method of data collection was developed, from which a variable called Annual Reliability was obtained. For this, the range of hourly values of three 
years was taken. The data collected were grouped hour by hour, which is, 1095 data were collected for each hour of the 24 hours of a day. This is done due to the seasonality of the weather conditions,

In order to define the work interval, the developed selection system was focused on the possibility of selecting a statistical reliability. Once this value is obtained, the percentile that statistically covers the chance of occurrence of this phenomenon during the year is selected. That is, if a user sets Xpercentage of annual reliability, the data taken will be the percentile $(100-\mathrm{X})$ of the total data for each hour of the day in order to ensure that at least the Xpercentage of the days per year the occurrence phenomenon is within the selected ones. Figure 11 shows an example of hour 9 with the $50^{\text {th }}$ and $30^{\text {th }}$ percentile, the $50^{\text {th }}$ represents the mean of value (that is a method widely used), the $30^{\text {th }}$ percentile that covers the $70 \%$ of observation. The contrast in the figures shows that the selection of the $70^{\text {th }}$ percentile covers more data than the $50^{\text {th }}$ one; however, in terms of design, the $30^{\text {th }}$ percentile will demand more elements.
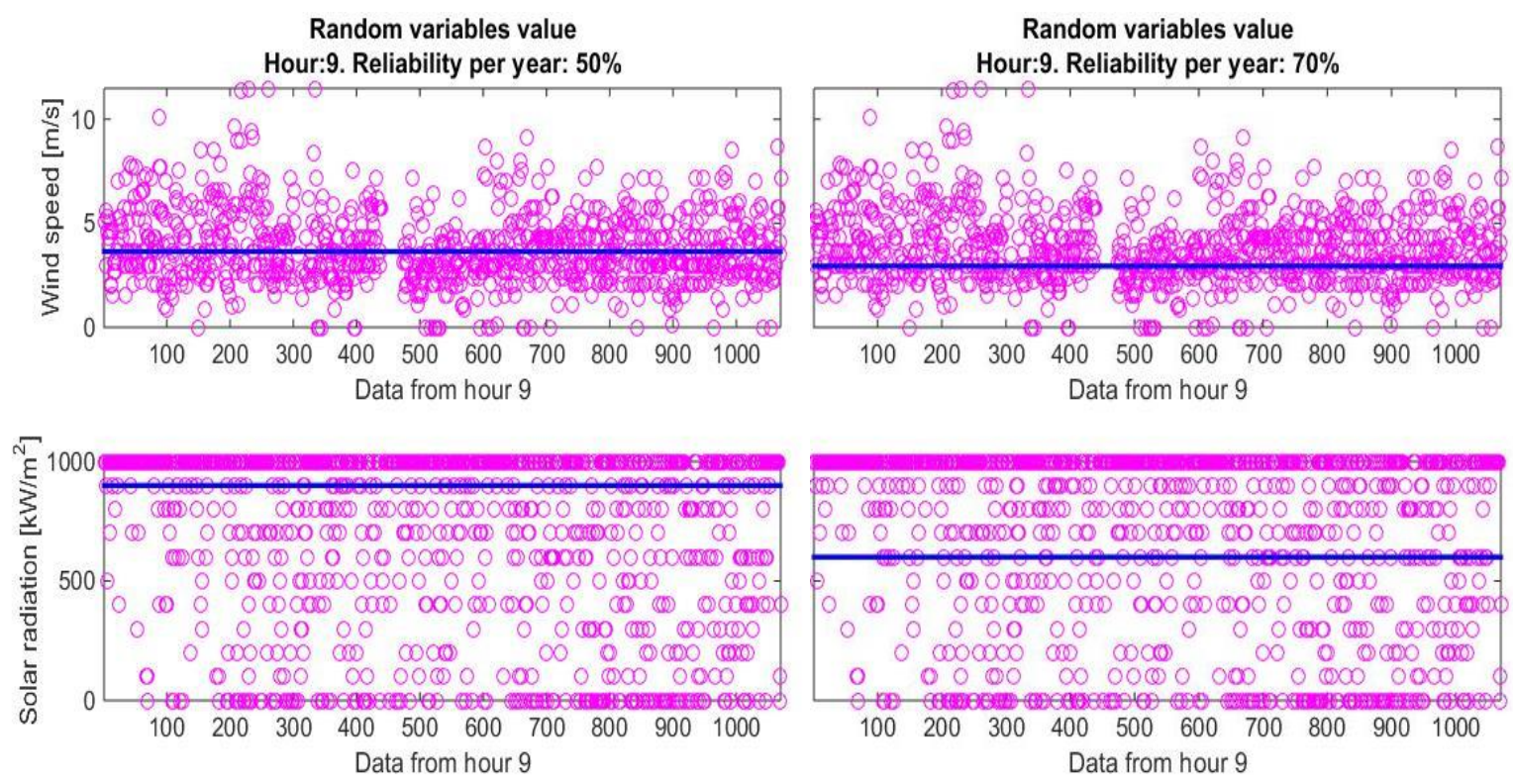

Figure 12. Wind speed and solar radiation of hour 9 with $50^{\text {th }}$ percentile in the left and $30^{\text {th }}$ percentile in the right image. Data from Barranquilla, Colombia. 


\subsubsection{Configurations}

Regarding the configuration of the system, there are four different possibilities of implementation: Configuration 1. Connected to the network with storage system, Configuration 2. Connected to the network with possibility of selling surplus energy, Configuration 3. Off-grid with storage system and Configuration 4. Grid-connected.

For each configuration, the energy generated by renewable systems is prioritized, always seeking to deliver all the required power to the load. For the case of the configuration 1 when there is surplus generated energy, the excess is stored in the batteries in case of availability, when the generated energy is not enough, the power stored in the batteries is used, and when this is not enough the missing power is bought to the grid, this is shown in Figure 13. In the case of configuration 2 when there is excess power generated, it is sold to the grid; when there is not enough power generation to supply the load, it is bought to the grid; this is shown in Figure 14. In the case of the configuration 4 when there is excess of power generated this is stored in the batteries if there is availability, but when it is not enough the energy stored in the batteries is used, this is shown in Figure 15. In the case of the configuration 5 when there is excessively generated power the load is fed without problem in case the grid is available due to the grid-connected inverters conditions, when there is not enough energy generated, the missing power is taken from the grid as shown in figure 15.

Figure 13, Figure 14 and Figure 15 show the operation of the system with each configuration in order to select the optimal elements for each case. For that, $P G=$ generated power from renewable energies $C B=$ batteries capacity 


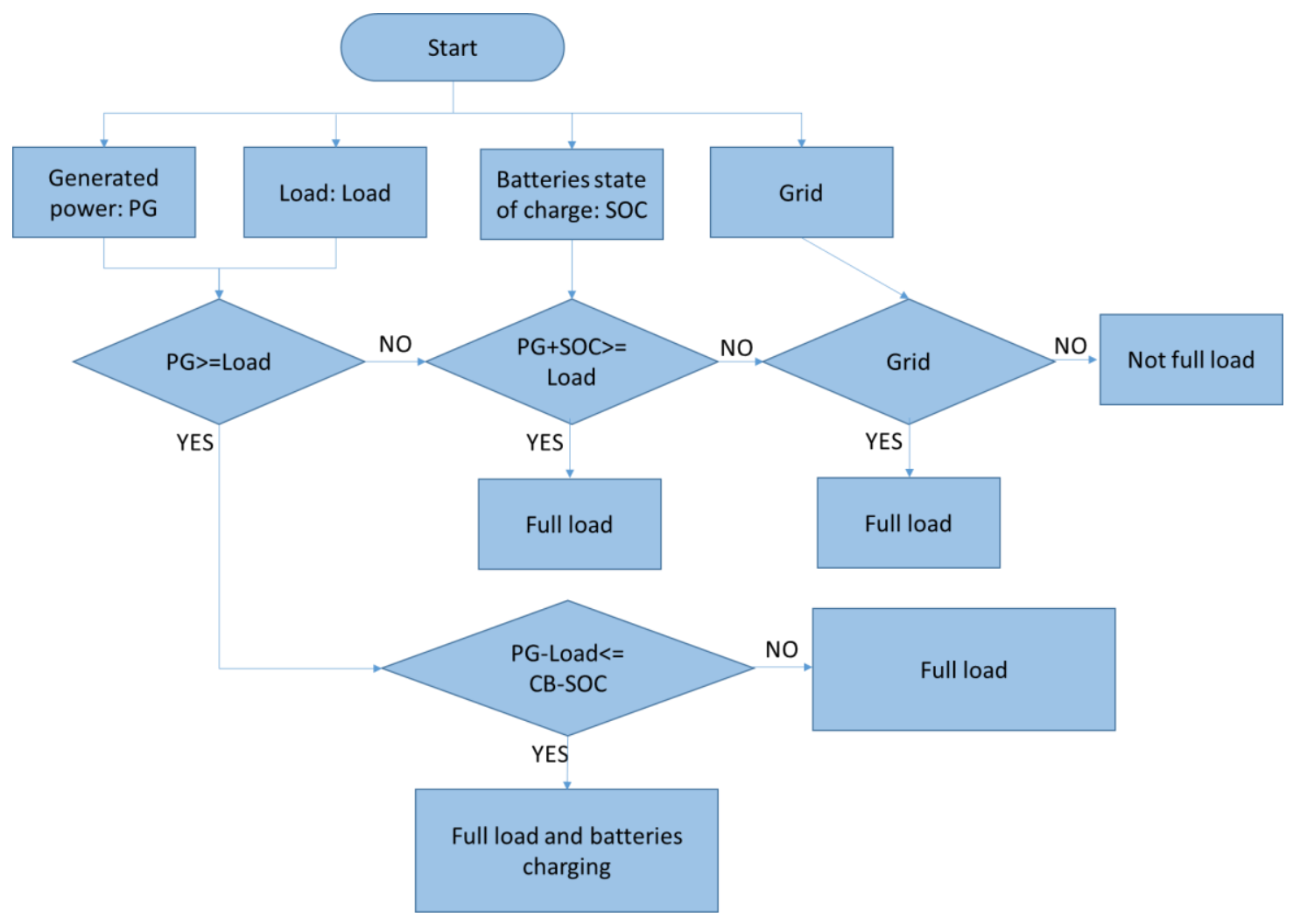

Figure 13. Configuration operation: Grid-Connected with batteries.

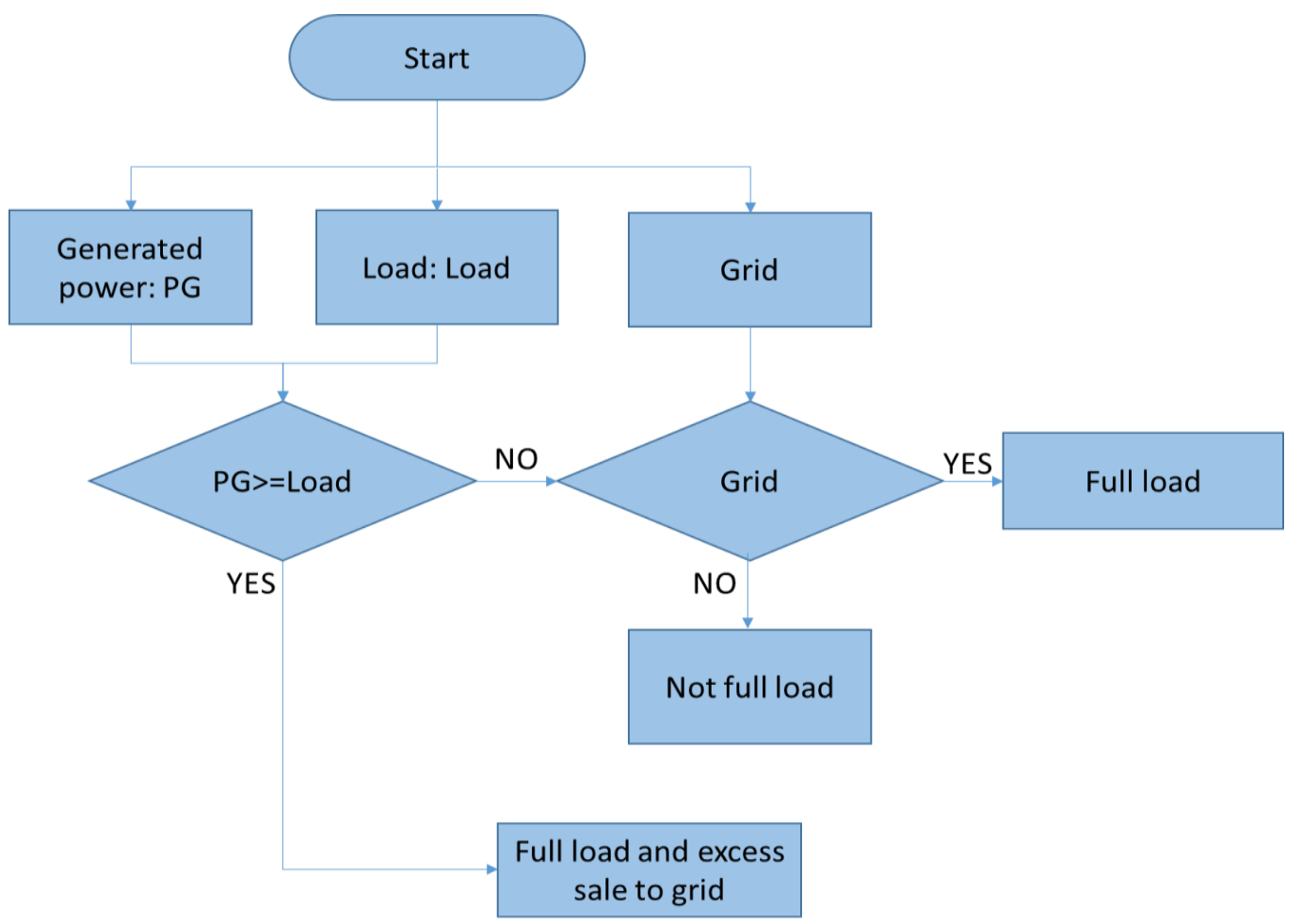

Figure 14. Configuration operation: Grid-connected with possibility to sale surplus energy 


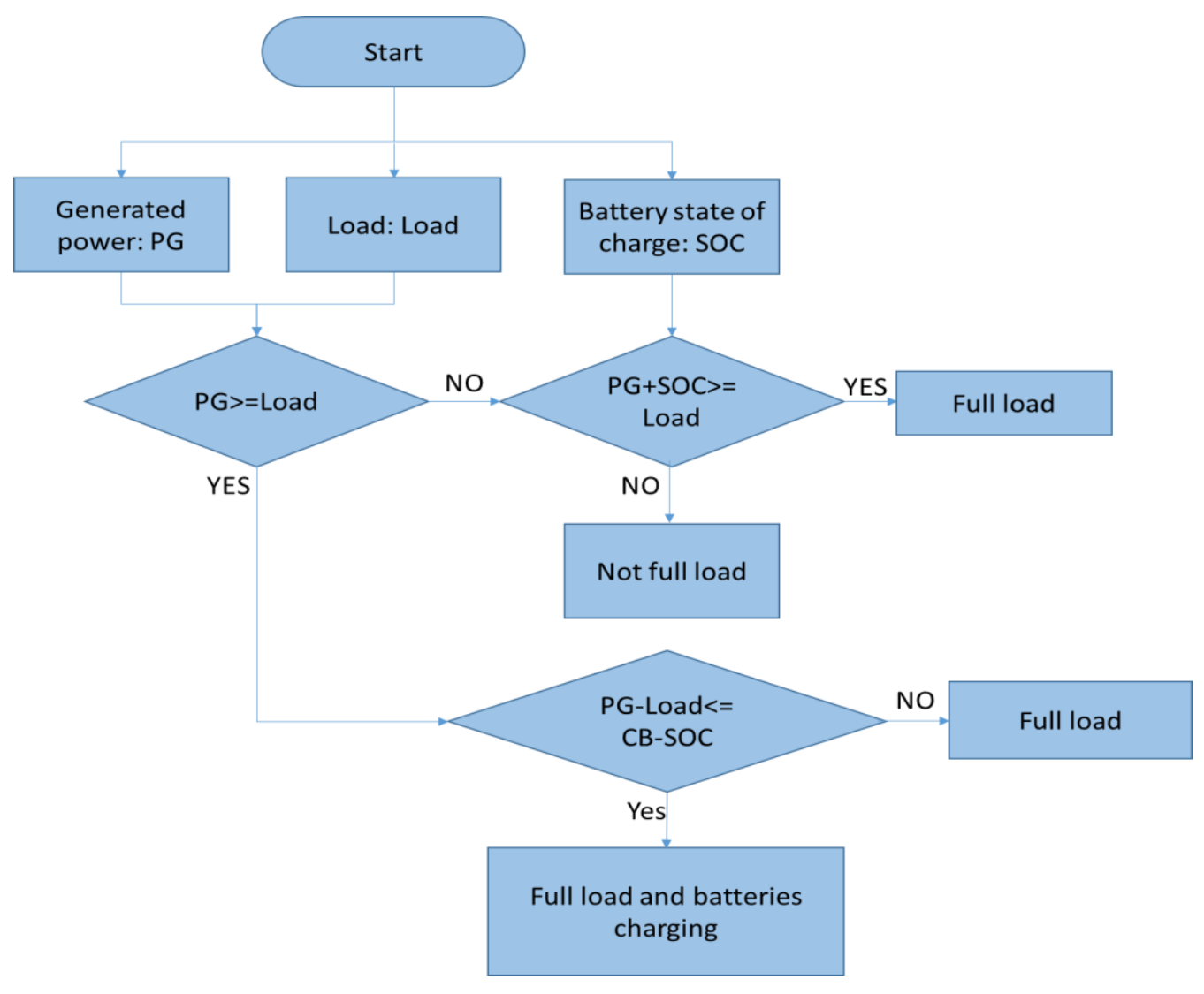

Figure 15. Operation of the Off-grid configuration: Wind-Solar-batteries

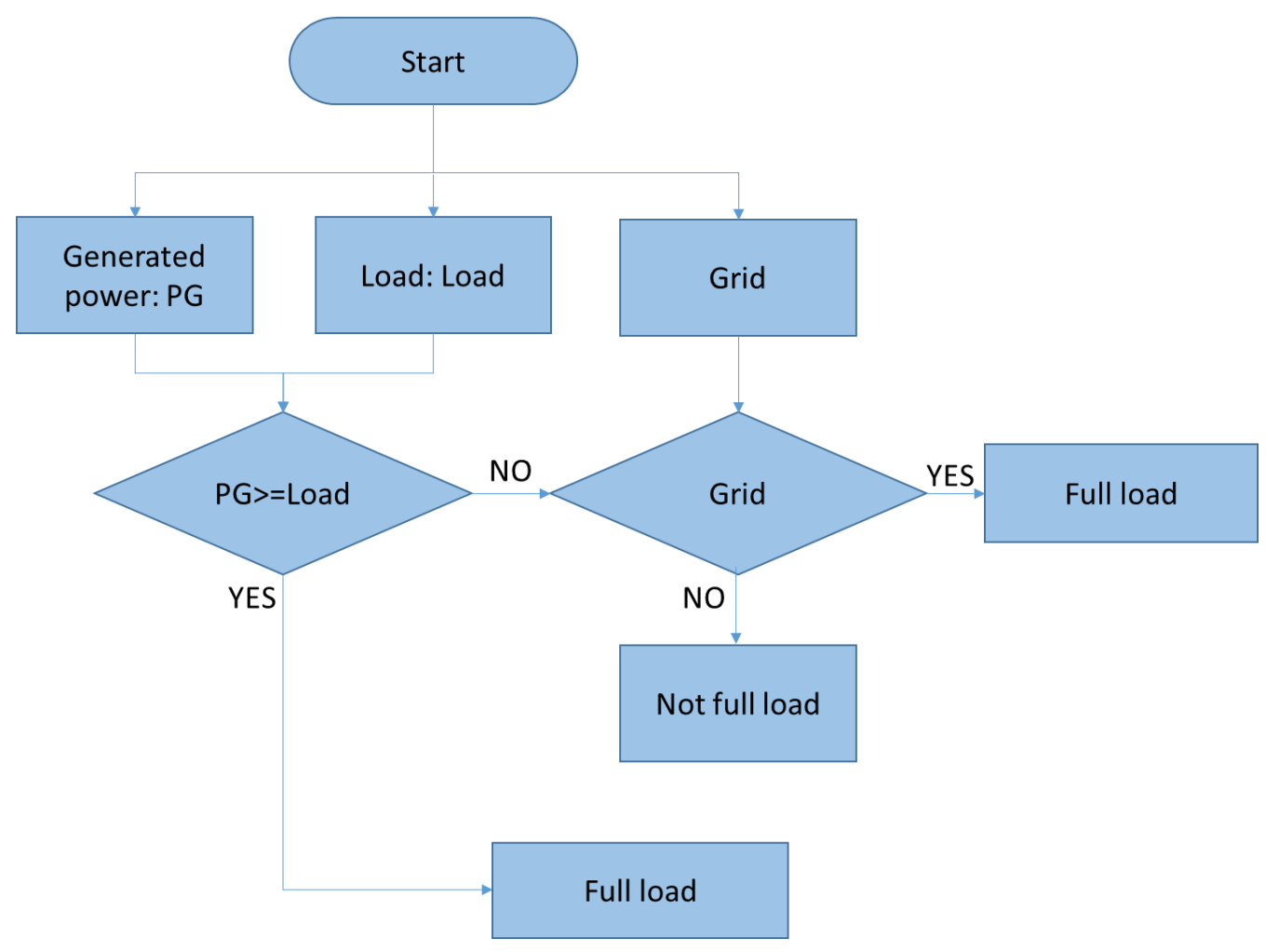

Figure 16. Operation of the Grid-connected configurationWind-Solar-Grid 


\subsubsection{System mathematical model}

According to the Table 1, Table 2 and Table 3, the selected mathematical models for solar panels, wind turbines and batteries are:

\subsubsection{Solar Panels}

The selected model was the most used in the studies analyzed. The governing equation that models the power generated by a solar panel is:

$$
P_{s p v}=N_{p a n} * V_{o c} * I_{s c} * f f
$$

And

$$
\begin{aligned}
& V_{o c}=V_{o c_{-} s t c}+k v *\left(T-T_{a m b}\right) \\
& I_{s c}=\left(I_{s c_{s t c}}+k i *\left(T-T_{a m b}\right)\right) * \frac{G}{1000} \\
& T=T_{a m b}+\left(\frac{N O C T-20}{800}\right) * G
\end{aligned}
$$

Where $N_{p a n}$ is the number of panels, $V_{o c}$ is open circuit voltage, $I_{s c}$ is short circuit current and $f f$ is the fill factor. $V_{o c \_s t c}$ is the open voltage measured under standard test conditions, $I_{s c_{-} s t c}$ is the short circuit measured under standard test conditions, $k v$ is the open voltage coefficient, $k i$ is the short circuit current coefficient, $\mathrm{T}$ is the standard temperature, $G$ is the global solar radiation $\left[\mathrm{W} / \mathrm{m}^{2}\right], T_{a m b}$ is ambient temperature $[\stackrel{0}{ }]$ and NOCT is Nomial Operating Cell Temperature.

\subsubsection{Wind Turbines}

The selected model was the most used in the studies analyzed. The governing equation that models the power generated by a wind turbine change depending of the wind speed as follows: 


$$
P_{w t}=\left\{\begin{array}{rl}
0 & v(t)<v_{c i} \\
P_{W T \text { max }} & v_{c i} \leq v(t)<v_{r a} \\
P_{r} \quad & v_{r a} \leq v(t) \leq v_{c o} \\
0 & v_{c o}<v(t)
\end{array}\right.
$$

And

$$
\begin{aligned}
& P_{W T \text { max }}=\frac{1}{2} r o * A * V(t)^{3} * C_{p} * n w * n m e c \\
& A=\pi * r^{2} \\
& V=v *\left(\frac{H \text { med }}{H t}\right)
\end{aligned}
$$

Where $P_{W T}$ is the power output of the wind turbine, $P_{W T \text { max }}$ is the maximum available output wind turbine power and $P_{r}$ is the nominal power generation. $r o$ is the air density, $v$ is the wind speed, $C_{p}$ is the efficiency of wind turbine, $n w$ is the inverter efficiency and nmec is the mechanical components efficiency, A is the area swept by the blades of the turbine and $r$ is the radio of blades, $\mathrm{V}$ is the wind speed at reference data, $\mathrm{H}_{\text {med }}$ is height of wind speed measurement, $\mathrm{H}_{\mathrm{t}}$ is the height of wind speed. $V_{c i}$ is the wind speed cut in, $V_{r a}$ is the wind speed rated power; $V_{c o}$ is the wind speed cut out.

\subsubsection{State of Charge of Batteries}

The state of charge of the bank batteries is:

$$
S O C=S O C_{-} *(1-r o)+P * n_{b a t}
$$

and

$$
P=P_{G}(t)-P_{L}(t)
$$

$P$ will be a negative value if the load exceeds the power generated from system. At any hour, the storage capacity is subject to the following constraints:

$$
S O C \max * D O D \leq S O C \leq S O C \max
$$


Where $S O C_{-}$is the initial value of the battery, $S O C_{\max }$ is the maximum value of power of the battery, $D O D$ is the maximum permissible depth of battery discharge, $P$ is the battery power, ro is the self-discharging rate and $n_{b a t}$ is the battery bank efficiency at charging state.

Due to the different configurations available, the inverters selected for each of them have different functions and configurations. In order to generalize the inverters, its mathematical model was performed only in function of its efficiency, (B. O. Bilal, et al., 2013) expressed the mathematical model of the inverter as shown in equation (5). For the Grid-connected configuration with bank of batteries and with possibility of sale excess to the grid, hybrid inverters are used; for the Grid-Off configuration, a Grid-off inverter is used and for Grid-Connected configuration, a Grid-tie inverter is used.

$$
P_{\text {out_inv }(t)}=p_{\text {in }} * n_{\text {inv }}
$$

Where Pout_inv is the output power of the inverter, $P_{\text {in }}$ is the input power of the inverter and $n_{\text {inv }}$ is the inverter efficiency.

\subsubsection{Optimization technique}

The genetic algorithm was the intelligent optimization technique selected to dimension the hybrid renewable energy system because according to Figure 4 that is the most common intelligent optimization technique used for hybrid wind-solar systems, also its adaptability and ability of solving problems with multiple solutions. The optimization seeks to determine the optimal: number of solar panels, wind turbines and batteries if the storage system is available. In addition, in dependence of the case study and the needs of the user, the optimization can also determine the optimal solar panel reference, wind turbine reference, and battery reference. Which means that the variables in the objective function could change in each case from two to six variables. 
Matlab environment was selected to code and implement the methodology with GA and GAMultiobjective tools. GA toolbox finds the minimum value of a function with only one fitness function using simple GA, this tool handles a row vector of numbers of length as variables, it also accept integer values as variables in the objective function, but it cannot be used linear equalities constraints when integer values are required. GAMultiobjective toolbox finds the minimum value of a function with two fitness functions using simple GA, this tool also handles a row vector of numbers of length as variables; however this one do not accept integer values as variables. The output of the GAMultiobjective tool is a Pareto Front for the fitness functions (MatWorks, 2015).

The fitness functions presented are selected according to Figure 7. However, cost selection was made according to money outflow values: both the initial cost of the system and the cost of the system over its useful life. The optimization approaches selected are:

1. Loss of Power Supply Probability (LPSP)

It refers to the power that cannot be supplied from the total demand, what is sought with this approach is to have a $0 \%$ probability of loss of charge. Mathematically it is expressed by equation (6).

$$
\text { LPSP }=\frac{\sum_{1}^{24} \text { Power supplied-Load Power }}{\text { Load Power }} * 100
$$

2. Initial Capital Cost (ICC)

Refers to the initial investment made by the user to purchase the elements of the system, it is sought to minimize this value to pay as little as possible following the established restrictions. The ICC is mathematically represented as shown in equation (7).

$$
\text { InitialCost }=C_{\text {pan }} * N_{\text {pan }}+C_{w t} * N_{w t}+C_{b a t} * N_{b a t}+C_{i n v}
$$


Where:

Cpan=Cost of each solar panel.

Npan=Number of solar panels.

Cwt=Cost of each wind turbine.

Nwt=Number of wind turbines.

Cbat=Cost of each battery.

Nbat=Number of batteries.

Cinv $=$ Cost of inverter.

3. Life cycle cost (LCC)

It refers to the total cost of the system during its life cycle. For this, values as life cycle of each element and its replacement in case of being less than the life cycle of the system, operation and maintenance of each element and initial cost of them are taken into account. Its mathematical expression is found in equation (8).

$$
L C C=C_{l p}+C_{l w}+C_{l b}+C_{l i n v}
$$

Where:

Clp=life cycle cost of solar panels. This is expressed according to equation (8.1).

Clw=life cycle cost of wind turbines. This is expressed according to equation (8.2).

$\mathrm{Clb}=$ life cycle cost of batteries. This is expressed according to equation (8.3).

Clinv=life cycle cost of inverter. This is expressed according to equation (8.4).

$$
\begin{aligned}
C_{l p} & =C_{s 1}+C_{s 2} \\
C_{s 1} & =C_{p a n} * N_{p a n} \\
C s_{2} & =\propto_{\text {po\&m }} * N_{\text {pan }} * \sum_{k=1}^{N_{y}}\left(\frac{1+\varepsilon_{p v}}{1+r}\right)^{k}
\end{aligned}
$$


Where $\varepsilon_{p v}$ is the inflation rate of the solar panel; $r$ is the real interest rate; $\propto_{\text {po\&m }}$ represents the maintenance rate per year; $\mathrm{N}_{\mathrm{y}}$ represents the horizon of the project in years and assumed equal to the lifetime of the solar panel.

$$
\begin{gathered}
C_{l w}=C_{w 1}+C_{w 2} \\
C_{w 1}=C w * N w * \sum_{x=1}^{X w}\left(\frac{1+\varepsilon_{w}}{1+r}\right)^{(x-1) l_{w}} \\
C_{w 2}=\propto_{w o \& m} * N p * \sum_{1}^{N_{y}}\left(\frac{1+\varepsilon_{w}}{1+r}\right)^{k}
\end{gathered}
$$

Where $\mathrm{X}_{\mathrm{w}}$ is the number of times the wind turbine requires replacement; lw life cycle year of the wind turbine; $\varepsilon_{w}$ is the inflation rate of the wind turbine; $\alpha_{\text {wo\&m }}$ represents the maintenance rate per year; $l_{\mathrm{w}}$ is the wind turbines lifetime.

$$
C_{l b}=C_{b a t} * N_{b a t} * \sum_{1}^{X_{b}}\left(\frac{1+\varepsilon_{b}}{1+r}\right)^{(x-1) l_{b}}
$$

Where $\mathrm{X}_{\mathrm{b}}$ is the number of times the batteries requires replacement; $\mathrm{l}_{\mathrm{b}}$ is the life cycle year of the battery and $\varepsilon_{b}$ is the inflation rate of the battery.

$$
C_{l i n}=C_{i n v} * \sum_{1}^{X} X_{i n v}\left(\frac{1+\varepsilon_{i n v}}{1+r}\right)^{(x-1) l_{i n v}}
$$

Where $C_{\text {inv }}$ is the initial cost of the inverter; $\mathrm{X}_{\text {inv }}$ is the number of times the inverter requires replacement; linv is the life cycle year of the inverter and Einv is the inflation rate of the inverter.

4. Loss of Power Supply (LPSP)

It refers to the hours of the day that the power of the load is not $100 \%$ supplied by the renewable system. The ideal is to have $0 \%$ of the power supply loss. Mathematically it is expressed by equation (9).

$$
L P S=\frac{\sum_{1}^{24}(\text { Power supplied }<\text { Load Power } \geq 1)}{24} * 100
$$

According to the GA toolboxes: GA and GAmultiobjective, one or two optimization approaches can be selected in order to optimize the hybrid system. 


\subsection{Final Remarks}

The sizing of a hybrid system has different topics to consider for optimal results. Starting with the inputs and configuration of the system, which are adaptable to the needs and requirements of each user; the meteorological conditions selected for the design, the mathematical models of the main elements and the selection and implementation of the optimization technique to be used. Figure 17 shows the general structure used for the optimum sizing of hybrid solar - wind systems adjusted to restrictions and requirements for different case studies.

Taking into account all of that, the next step is the development of the tool that will allow users to interact with the system in a simply and efficient way. For this, the implementation of the steps developed in this chapter and the development of the computational tool for its use is shown in the following chapter.

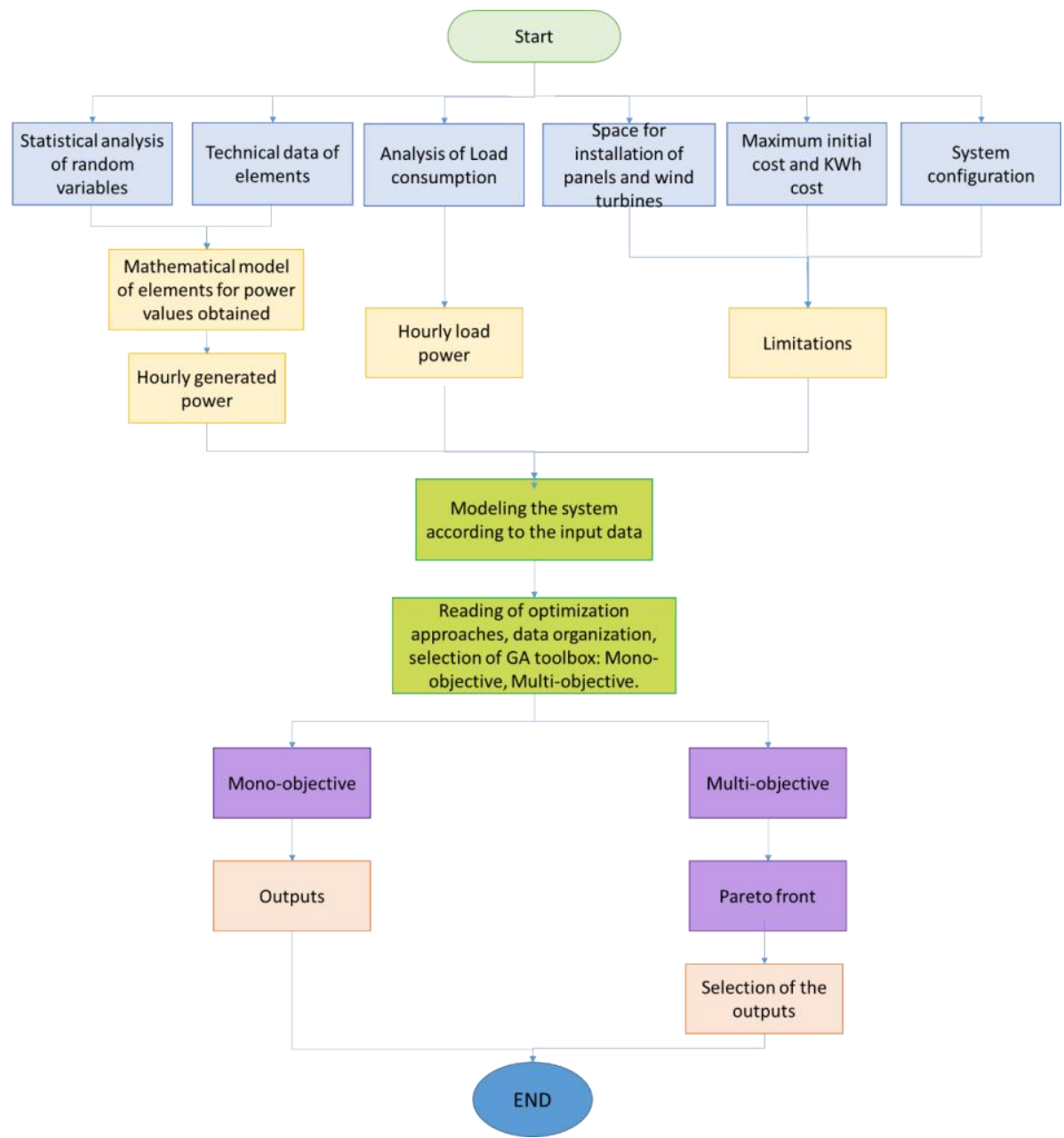

Figure 17. General structure of the methodology 


\section{Chapter 5}

\section{Implementation of a sizing approach for hybrid renewable energy system}

This chapter presents the application of the proposed sizing approach for hybrid renewable energy system with solar and wind energy.

\subsection{Developed Computational Tool}

In order to develop a computational tool that allows a feasible sizing optimization, the MATLAB GUI application was used for its implementation. GUIs (also known as graphical user interfaces or UIs) provide a graphical language software applications, eliminating the need to type commands in order to run the application.

When the user decides to use the developed computational tool for sizing hybrid renewable energy systems, the first thing to define is in which currency the sizing will be performed, the two options allowed are USD Dollars and Colombian Pesos COP. To do this, a window is displayed like the one shown in Figure 188.

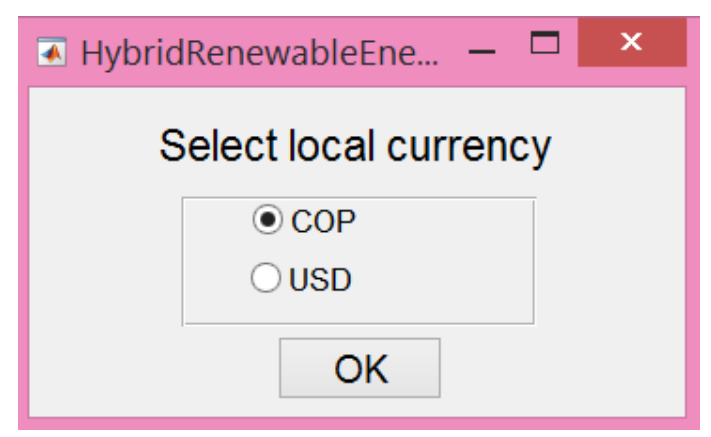

Figure 18. Selection of local currency in the developed system. 
For the correct operation of this tool with updated data, due to the fact that the data found of trade and price of the elements is mostly found in dollars, the stored data in this software is saved in this currency, so, when the user selects COP, all values as inputs given by the user in COP are internally passed to USD. For this, the current value of a USD in COP is downloaded from the web page: http://www.dolarcolombia.com/ and the change of currency is made. However, when the system returns the responses to the user, it passes from USD to COP so that the user receives the values in Colombian pesos.

After defining the currency and clicking in OK button, the main window of the system is displayed and in this, the user must select and/or introduce the system inputs, as shown in Figure 19. The system has default values but they can be modified. At the end of filling all the input blanks, the user must press the Execute button to start the process of sizing the hybrid system according to his needs and requirements.

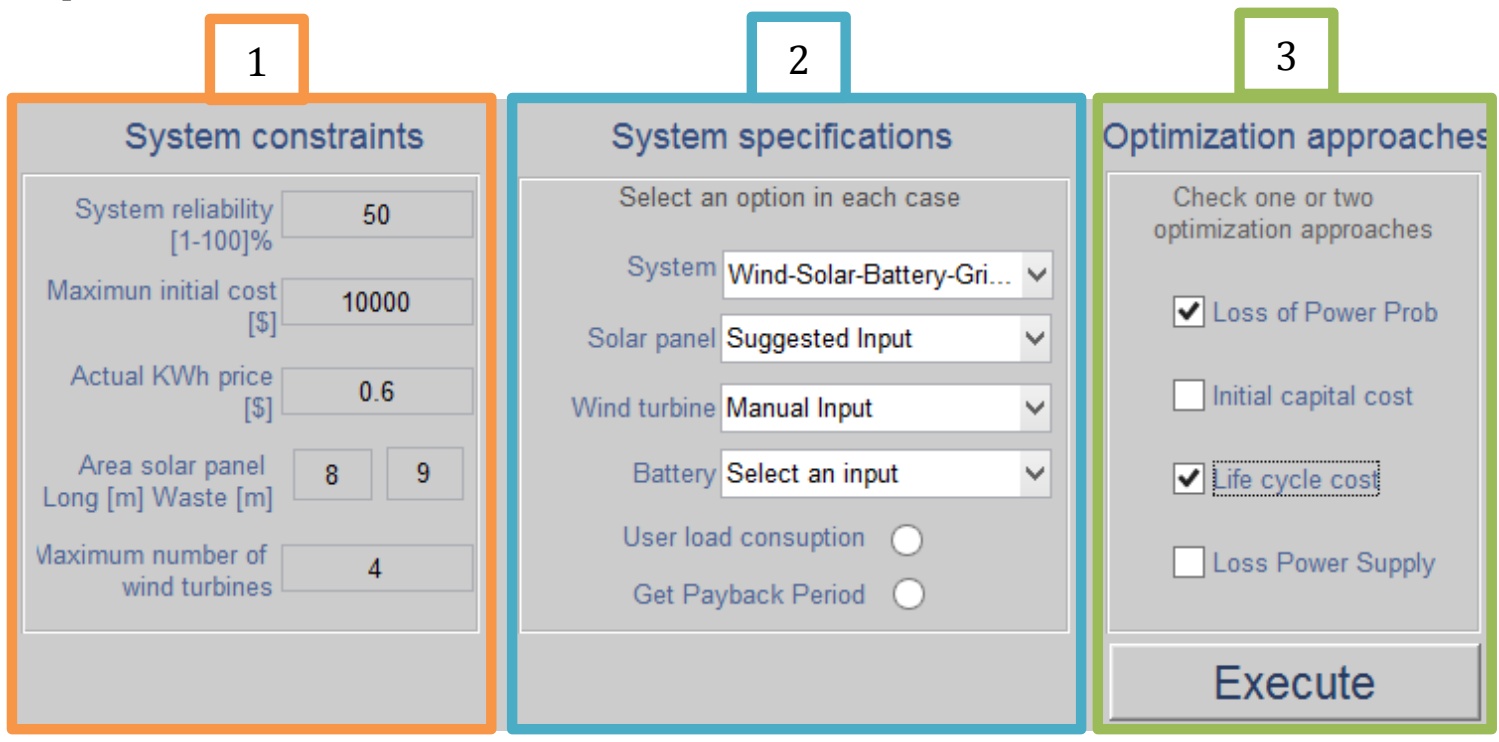

Figure 19. Inputs of the developed system.

Figure 19 shows three large blocks, the first of which refers to the constraints of the system. For that, the minimum reliability percentage in the hybrid system to be implemented is required. This reliability value is annual and with that, the statistical process of selecting a 24-hour interval data from the climatological random variables of the system starts; in this case, it is recommended that the reliability 
values be in a $25-75 \%$ range since values outside this range are considered isolated data. After that, the maximum initial cost value that the user is able to pay for the system. It is important to note that according to the specifications presented, these restrictions can or not be always met because the values can be outside a common range, for example, a user can request to have a $70 \%$ of reliability of the system during the year with only 100 dollars. Then, the KW/h cost handled by the network operator is required in order to carry out the purchase and sale of energy if its possible. Also, the area available for the placement of the solar panels so that the amount of them is limited, as well as the maximum amount of wind turbines.

The second block, called system specifications, requires the configuration of the system to be implemented. The options offered are four: 1 . Wind-solar-battery-grid, 2. Wind-solar-grid-sale excess, 3. Wind-solar-battery and 4. Wind-solar-grid. User must select the option from the list displayed. After this, the reference of the solar panels, wind turbines and batteries must be selected; for this, there are three options in each case: Suggested input, which refers to allowing the software to optimize the reference to use, this is done according to some backup references saved previously (these references are shown in Appendix). Manual input, when the user selects this input, a window like the one shown in Figure 20 is displayed; in this, the user must enter the data of his selected reference. Select an input, when the user can choose a reference from those stored in the system; to do this, the option must be selected from a list in a displayed window that shows the possible selection references.

After these options, User Load Consumption can be found. By pressing the button adjacent to this name, a window is displayed where the user must enter in a $24 \mathrm{~h}$ interval, the load data during a typical day of consumption. Next is Get Payback Period, these data are requested to calculate the payback period in years. For that, interest rates in the location where the user is, as well as the operation and maintenance rates of solar panels, wind turbines and batteries are requested. 

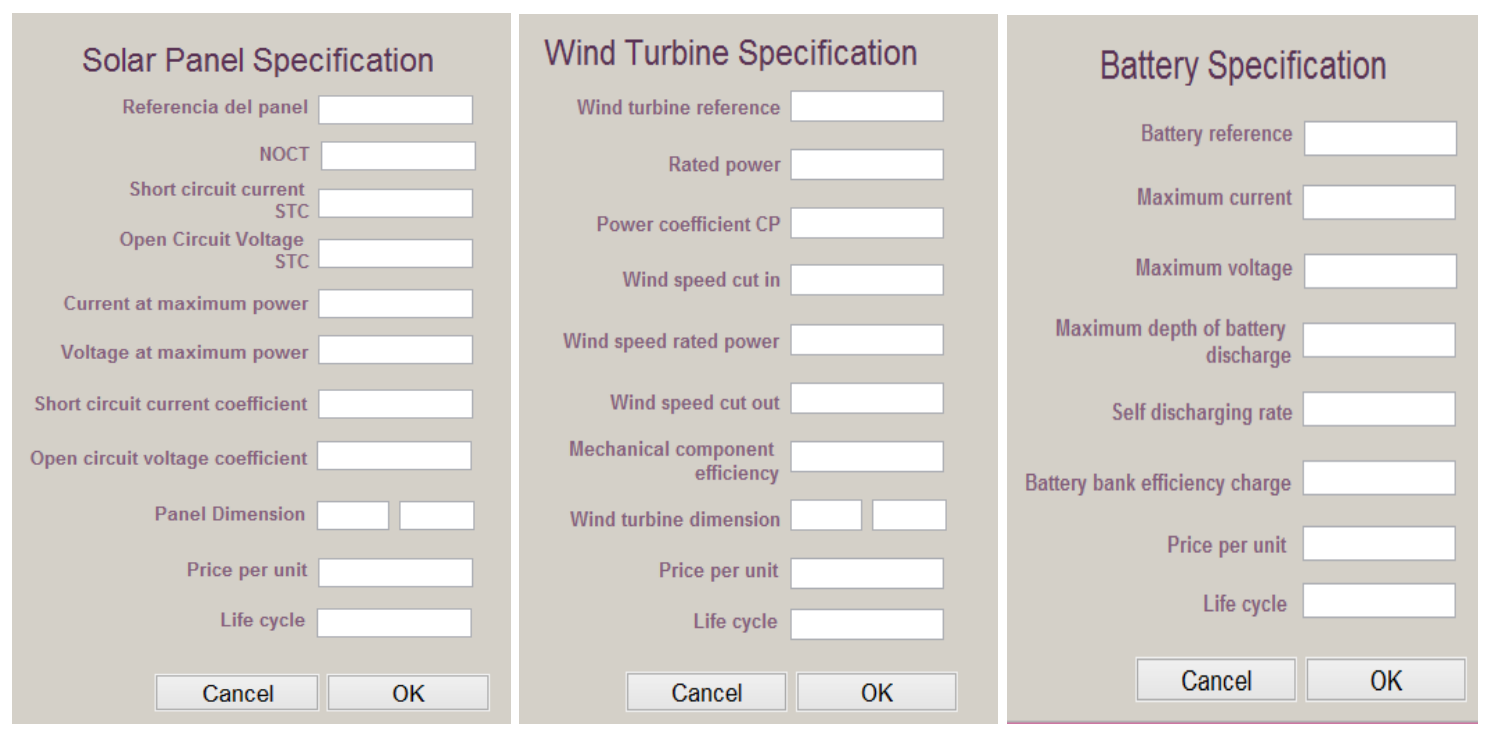

Figure 20. Manual input displayed windows for solar panel, wind turbine and battery.

The third block of entries is the block of optimization approach, in this, the user has the possibility to select one or two optimization approaches in order to dimension the system. The options presented are four: Loss of Power Probability (LPP), Initial Capital Cost (ICC), Life Cycle Cost (LCC) and Loss of Power Supply Probability (LPSP).

Figure 21 shows the graphical interface with which the user will be related while the sizing of the system is performed. As explained above the first thing to do is to enter all the entrances and press the execute button. Next, the user must wait for the system to perform the sizing and output the results. Figure 18 is divided into boxes in order to allow the explanation of what is shown on it. 


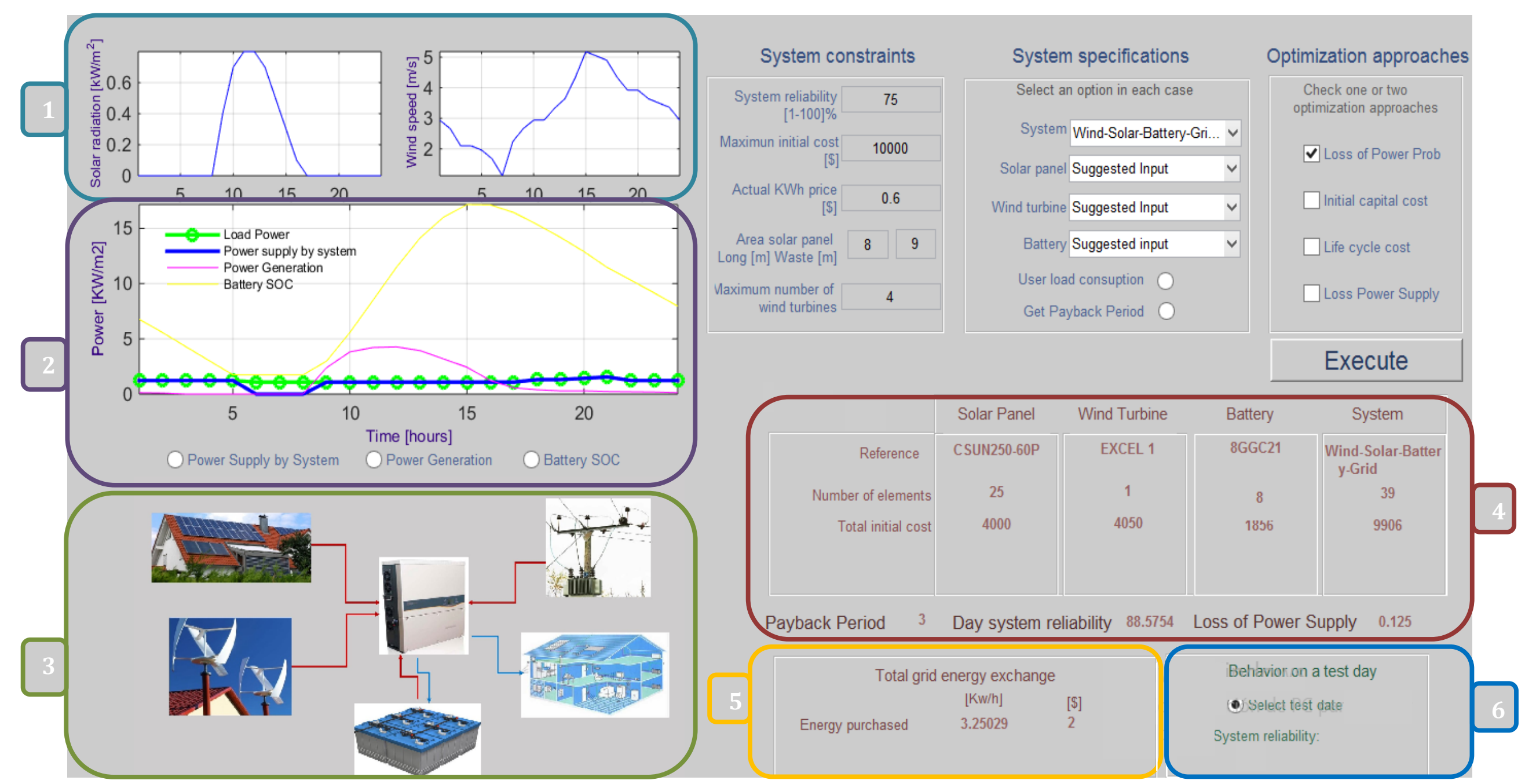

Figure 21. Graphical software interface 
Box 1 shows the selected values of solar radiation and wind speed according to the selected reliability; these are displayed in a $24 \mathrm{~h}$ interval and are expressed in $\mathrm{KW} / \mathrm{m}^{2}$ and $\mathrm{m} / \mathrm{s}$ respectively. Box 2 shows the behavior of the system with the selected values. The green line is the load introduced by the user, the blue line, is the power delivered by the system to the load, the magenta shows what is generated by the solar and wind sources in each hour and finally the yellow one that shows the behavior of the batteries. Below this graph, the names of the three lines are shown and a button next to them is available to click; that is in order to enable or disable them in the graph if required. Box $\mathbf{3}$ shows the system components and power flow according to the user's selection.

Box 4 shows the reference, quantity and initial cost of each element and finally the total elements and initial cost of the system. If life cycle optimization has been selected, the life cycle cost values of the system are displayed as shown in Figure 22. Under that, the Payback period of the system in years, the reliability per day and the loss of power supply value are presented. Box $\mathbf{5}$ shows in case of being enabled the purchase and sale of energy daily values in $\mathrm{KW} / \mathrm{h}$ and cost of these transactions. Finally, Box 6 allows a test to be run with a specific day of the year selected.

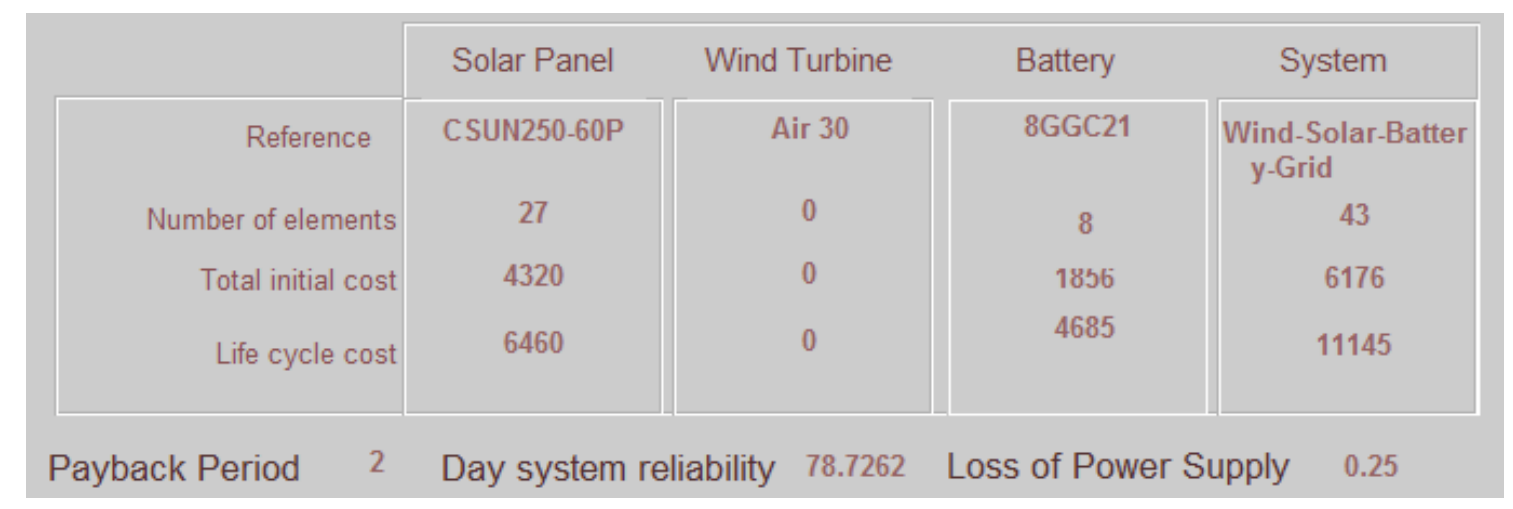

Figure 22. Output with life cycle cost optimization approach selected.

\subsection{Modeling of different configurations}

It has been mentioned that there are four (4) different possible configurations of the system and Figures 11, 12, 13 and 14 show their operation. The programming of each of the configurations was different, due to the elements and restrictions; 
however, the main idea is the similar. For the general operation of the system, different variables were defined for power values. For this

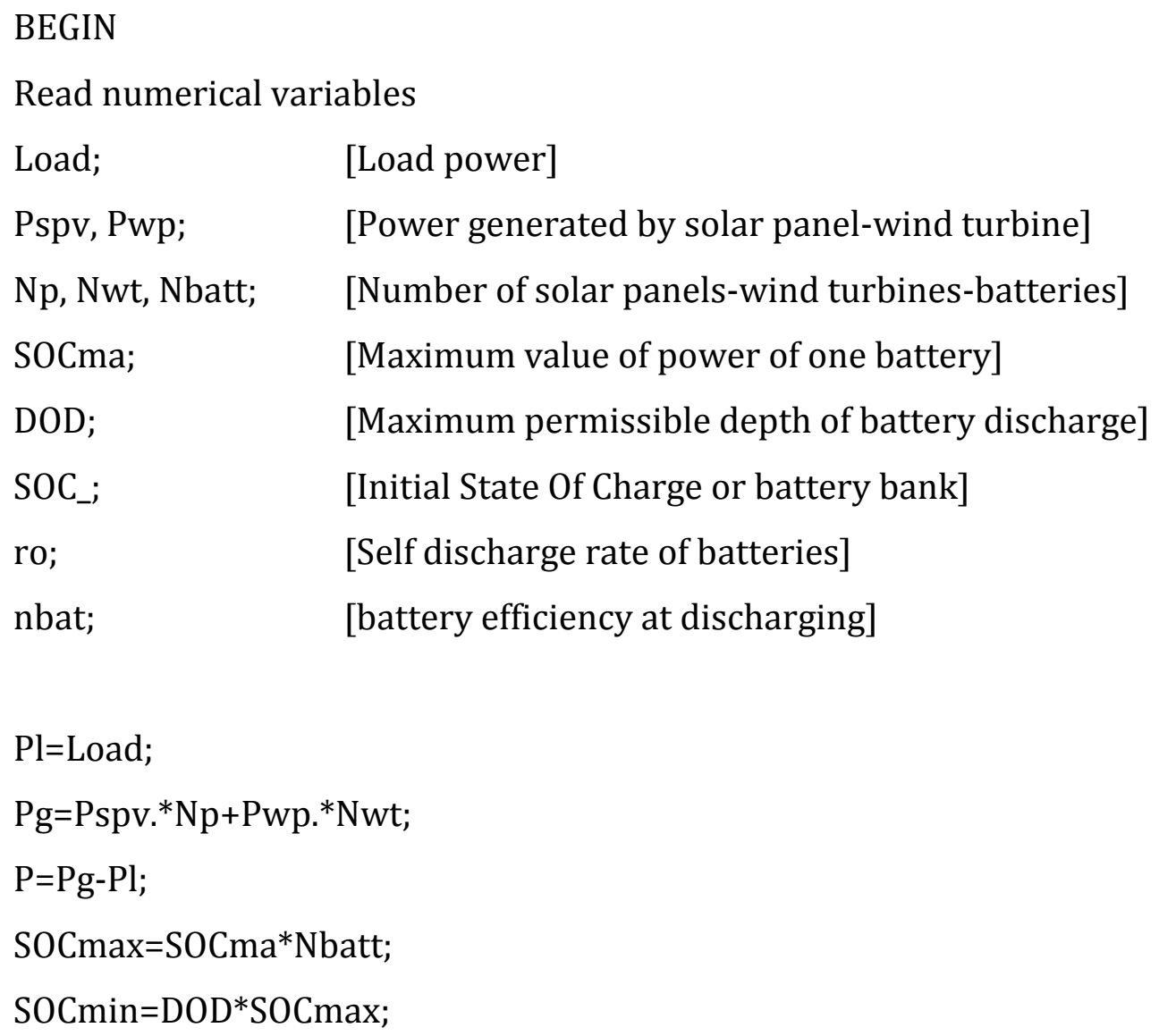




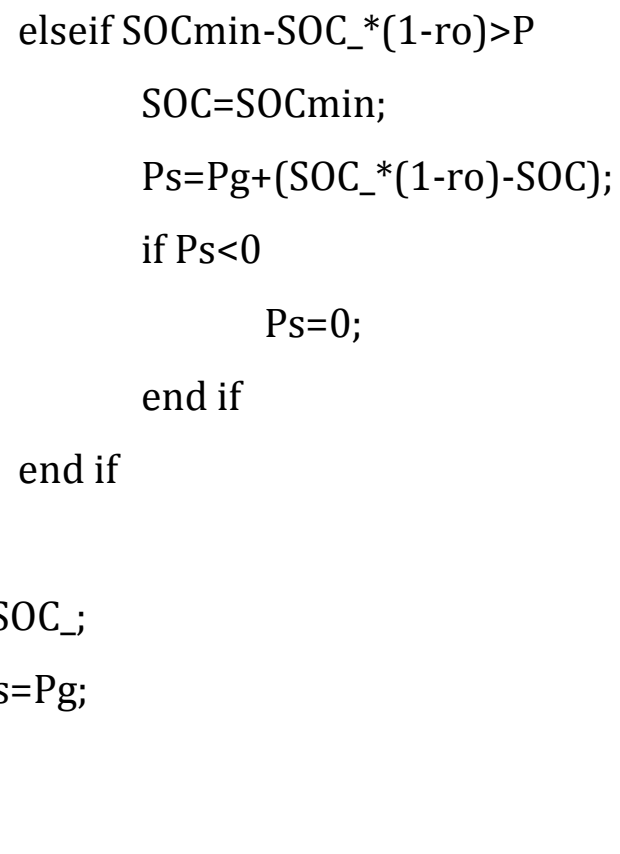

This process is repeated for the 24 hours of the day. In order to know the initial power of the storage system, the process is repeated for other 24 hours taking into account the last State of Charge of batteries at the period 24. When there is no storage system available, the process is the same, assuming that the SOC is always Cero and the batteries cannot be fulfilled.

\subsection{Genetic algorithm programming}

The optimization for the sizing of the solar-wind hybrid system was carried out using genetic algorithms. For this, GA and GAMultiobjective toolbox of Matlab were used. The parameters of the optimization are variable for each particular case. The objective function is variable according to the selection of the user, in this case, one or two optimization approaches can be selected (mono-objective or multi- Objective optimization) from the four presented. The number of variables changes depending on the configuration of the system (whether or not with batteries) and the optimization of the references of the elements. Therefore, it is possible to have between two (2) and six (6) variables for each case. Regarding the restrictions, these are also dependent on the configuration that is used, of the variables set by the user 
as maximum initial cost and minimum reliability and of the physical conditions for the installation as the maximum number of solar panels and wind turbines.

\subsubsection{Mono-objetive optimization}

The Matlab GA toolbox was used to perform the mono-objective optimization. In this step, variables, objective function and constraints must be defined.

The objective function was previously selected by the user, therefore this does not represent a big problem; in the case of the variables, depending of the user selection, a vector called is created where the selected variables are saved. All variables in this optimization are integer, so a vector called IntCon that contains the number of variables must be created; in addition to this, the upper $(u b)$ and lower $(l b)$ limits vectors must be defined in order to be introduced into the genetic algorithm programming. The minimum number of elements can be zero, but in terms of the optimization of references, the minimum number must be one since a reference should always be selected. For the upper bound, the number of panels is given by the user's available area and the dimensions of the panels, the maximum number of wind turbines is given by the user as input and the maximum number of batteries was selected as eight (8) since this system is for loads less than $5 \mathrm{~kW} / \mathrm{h}$.

System constraints are given in terms of minimum reliability and maximum cost, for this, a function called ConstraintFunction was created for acquiring those values. The only case in which these two restrictions are not available is when the system is not connected to network, since the system is required to have a $100 \%$ daily reliability at any cost. It is important to note that when working with the Matlab GA toolbox with mixed-integer problems, it is not possible to have equality in equations or in-equations as constraints.

The GA toolbox allows modifications to the genetic algorithm options; however, when talking about integer variables, it has some restrictions in them, so that the optimization was proposed with the following parameters: 
Modifiable:

CrossoverFraction $=0.7$;

PopulationSize $=28^{*} n$ Vars;

Generations $=700 * n$ Vars;

FunctionTolerance=1e-9;

MaxStallGenerations $=500 * n$ Vars;

Not modifiable:

Binary tournament selection function (SelectionFcn option)

To obtain integer variables, use special creation, crossover, and mutation functions.

The modifiable variables were selected by doing tests trying to obtain low execution time and similar responses in each run, which would indicate that the process was being performed correctly and always converged to the same solution. While the genetic algorithm is running, the user has the possibility to observe how the best individual converges. Figure 233 shows the windows that appears in the user interface when the optimization is in process. The graphic in the top indicates in black the best results obtained and in blue the mean of results of the population that has been analyzed. The graphic in the back shows the variables with which the best result was obtained. The figure is interactive and is changing as the result changes. The answer in the graph is with negative values since the GA function of Matlab seeks to minimize results and not to maximize them, and in the case of efficiency, this one should be maximized.

With all optimization parameters defined, the $G A$ toolbox is called as shown below [x, fval] = ga(FitFcn, nVars, [], [], [], [], lb, ub, ConstraintFunction, IntCon, options);

The outputs are "x" and "fval", represent the values of the variables and the response obtained with them. 


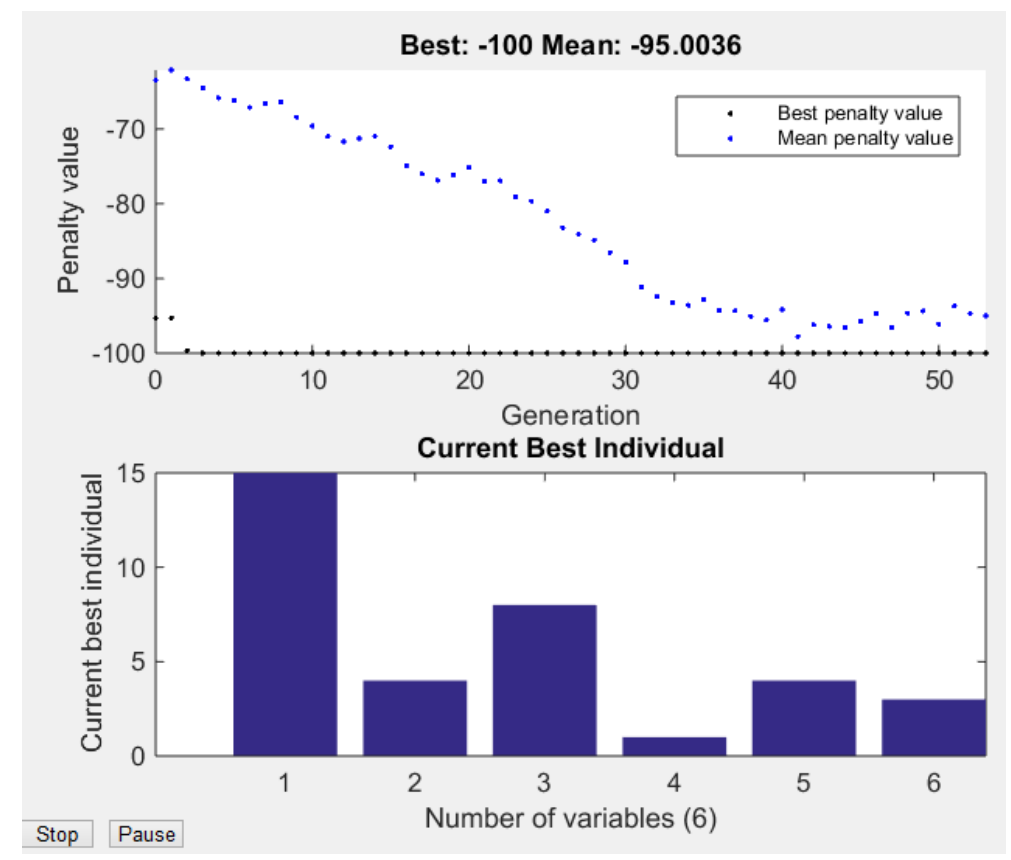

Figure 23. Interactive window showing the convergence of optimization.

\subsubsection{Multi-objective optimization}

The Matlab GAMultiobjective toolbox was used to perform multi-objective optimization. For this, the variables, objective function and constraints must be defined.

Similarly, it was done with the mono-objective optimization, the objectives functions were previously selected by the user; in the case of the variables, depending of the user selection, a vector called $n$ Vars is created where the selected variables are saved. In addition to this, the upper $(u b)$ and lower $(l b)$ limits were created. System constraints are also given in terms of minimum reliability and maximum cost; for this, a function called ConstraintFunction was created for acquiring those values. It is important to note that when working with the Matlab GA Multiobjective toolbox, integer variables are not accepted.

In this case, the structure of the source code of the genetic algorithm for multiobjective solutions was studied. The first action of the algorithm is review all the optimization parameters; from there, it randomly generates the values of the initial population. The first change was made at this stage, since the randomly generated 
values were not required integers. For this, the generation of the initial population was modified defining for the initial population only integer values and verifying that they were within the established limits. Next, changes were made to the Crossover and Mutation functions in the GAMultiobjective toolbox of Matlab, in order to always find integer values as solutions. It is important to note that this solution was performed for this particular optimization since all variables are always integers.

The GAMultiobjective toolbox allows modifications to the genetic algorithm options, the selected ones are shown below

- PopulationSize $=84 * n$ Vars;

- Generations $=200 * n$ Vars;

- MaxStallGenerations=100*nVars;

- FunctionTolerance=1e-8;

- 'CreationFcn',@gacreationuniform,...

- 'SelectionFcn', $\{\{@$ selectiontournament,2\}\},...

- 'CrossoverFcn',@crossoverintermediate,...

- 'MutationFcn',@mutationadaptfeasible,...

The modifiable variables were selected by doing tests trying to obtain low execution time and similar responses in each run, which would indicate that the process was being performed correctly and always converged to the same solution. The GAMultiobjective presents a Pareto Front as a solution of the system; the user can observe how it is finding the Pareto front while the system performs the optimization in Figure 24.

With all optimization parameters defined, the GAMultiobjective toolbox is called as shown below

$[\mathrm{x}, \mathrm{fval}]=$ gamultiobj(FitFcn,nVars, [], [], [], [], lb, ub, ConstraintFunction, options); 
The outputs are " $\mathrm{x}$ " and "fval", which represent the vector values of the variables (Pareto front) and the response obtained with each of them.

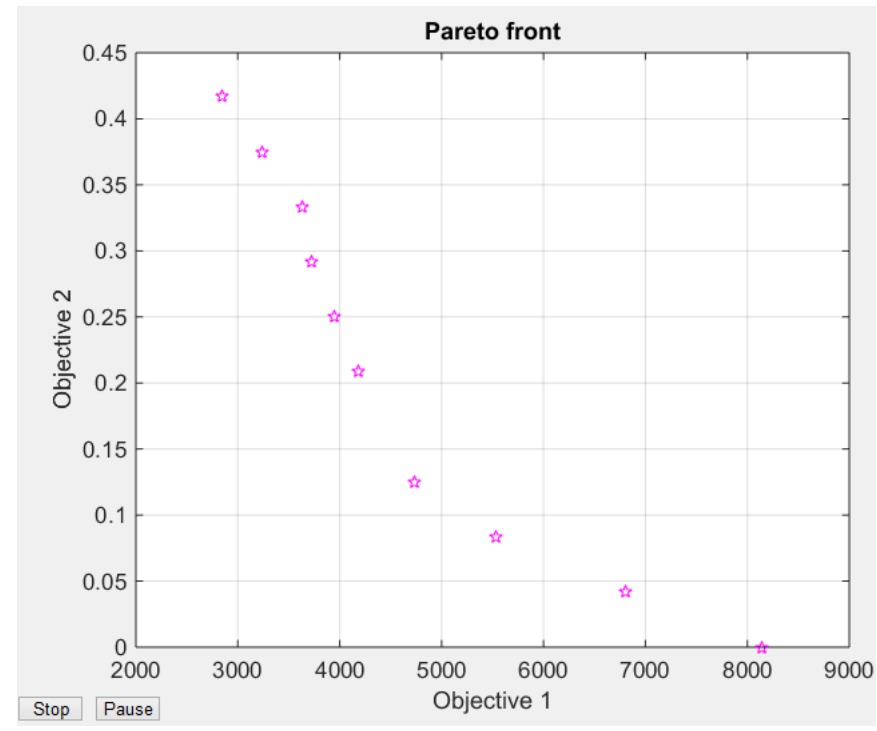

Figure 24. Interactive window showing the Pareto Front obtained

\subsection{Economic analysis}

The economic analysis seeks to give information about the initial and life cycle costs of the system and the time of recovery of the investment. Equation (7) shows the initial cost of the system and equation (8) shows the calculation of the system life cycle cost.

$$
\begin{aligned}
& \text { InitialCost }=C_{\text {pan }} * N_{\text {pan }}+C_{w t} * N_{w t}+C_{b a t} * N_{b a t}+C_{i n v} \\
& L C C=C_{l p}+C_{l w}+C_{l b}+C_{l i n v}
\end{aligned}
$$

For the time of recovery of the investment, the concept of flow diagram was used to visualize how the investment flows through the time. The analysis period of the system was performed annually and the flow diagram of the system is presented in Figure 25.

For this, ICC is the value of the initial cost of the system, AE are the annual profits generated and 0\&M are the annual operation and maintenance costs of the system. RCB is also observed which is the replacement cost of batteries that are assumed to have a lifetime of X years and RCW is the replacement cost of wind turbines that 
have an $\mathrm{X}+1$ life cycle years. For this example, the lifetime of the system is assumed to be $2 \mathrm{X}+3$ and the life cycle of the panels is equal to or greater than this value.

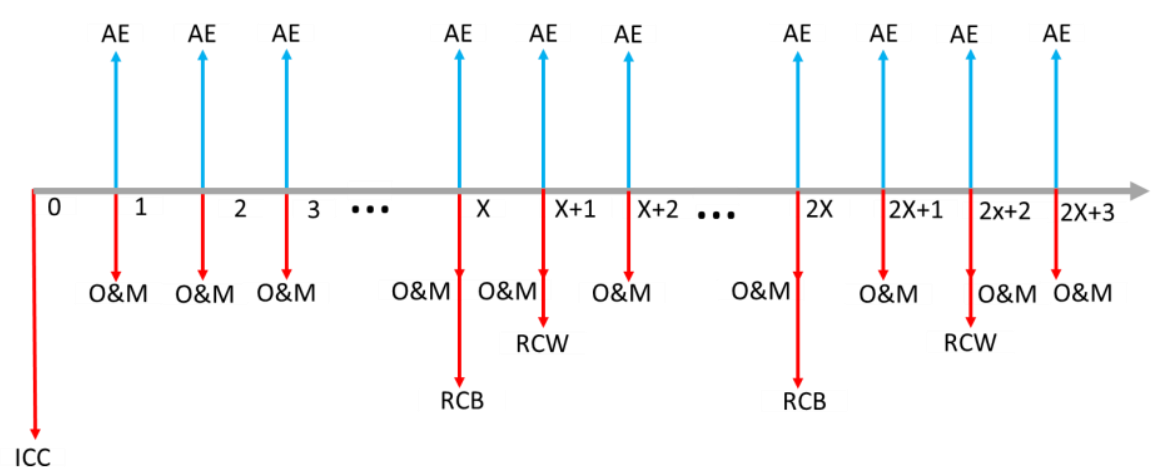

Figure 25. Flow diagram in the system life cycle time.

The ICC value was obtained according to equation (8); The O\&M values were obtained as a result of the sum of the annual operation and maintenance costs of the solar panels, wind turbines and batteries that were requested to the customer as input to the system. The AE value was calculated as shown in equation (10).

$$
A E=\sum_{1}^{24} \frac{\text { Power supplied by system }\left(P_{S}\right)}{1000} *\left(365 * \frac{\text { Reliability per year }}{100} * \text { Price KWh }\right)
$$

This value is the saving of the KWh that were supplied by the system to the load on the days that the annual reliability statistically ensure.

With these values, the flow chart was used to calculate the years of return on investment. For this purpose, all future values were taken to present value using equation (1) and the outputs with the entries seeking to obtain a VPN equal or less than zero, which means that the user has recovered the money invested.

$$
P=\frac{F}{(1+i)^{n}}
$$




\subsection{Final remarks}

The proposed system for optimizing the sizing of the renewable energy hybrid system with solar and wind energy was developed using the tools of Matlab GA and GAMultiobjective; however, there was a need to modify them to fit the needs of this system.

To generate benefit to the study presented, the development of an interactive tool is important. The way it is adaptable to the needs and limitations of each case study and its technological development becomes vital in this type of studies because the conditions are always variables.

The next chapters present experimental results to evaluate the development of the sizing system presented; in addition, conclusions and future work are shown. 


\section{PART III}

\section{EXPERIMENTS}

RESULTS AND

CONCLUSIONS 


\section{Chapter 6}

\section{Analysis of the Experimental Results}

This chapter presents the discussion and analysis of the empirical experiments and testing that have been carried out for the proposed test beds. The results depicted in this chapter demonstrate the utility, feasibility and reliability of the overall proposed approach presented in the previous chapters.

\subsection{Experimental Results}

The focus of the experiments was established in order to demonstrate the independence and variability of the results of the system against the different case studies. For this, we compare the results obtained by the system implemented in this thesis: Hybrid Renewable Energy System (HYRES) with the results of the commercial software HOMER in order to show the utility, feasibility and reliability of HYRES. In addition, a comparison of the results obtained with HYRES according to different input variables is shown.

In order to compare the results delivered by the HYRES with the results obtained by HOMER, it is important to emphasize that HOMER has the particularity of minimize the Net Present Value (NPV) of the system in every optimization case. In addition, other variables of HYRES are not possible to modify using HOMER as the optimization of the elements references and the initial maximum cost.

Consequently, the following characteristics are defined for the analysis of results: Variant Factors:

1. Reliability: establishes the annual reliability of the system with values of $50 \%$, which is equivalent to the average and $75 \%$ according to the suggestion presented in section 5.1 . 
2. Location: three different locations were established: Miami-US, BarranquillaColombia and Fortaleza-Brazil due to their different positions.

3. User demand: two user demands were selected with differences in the demand curve and daily consumption. The selected demand profiles were taken from (Essa, 2004), as different users. Values are presented in Figures 26 and 27.

4. System configuration: Two configurations of the system were selected, Configuration 1: Solar PV - Wind Turbines - Batteries - Grid connection (non possibility of selling energy to grid) and Configuration 2: Solar PV - Wind Turbines - Grid connection (possibility of selling energy to grid).

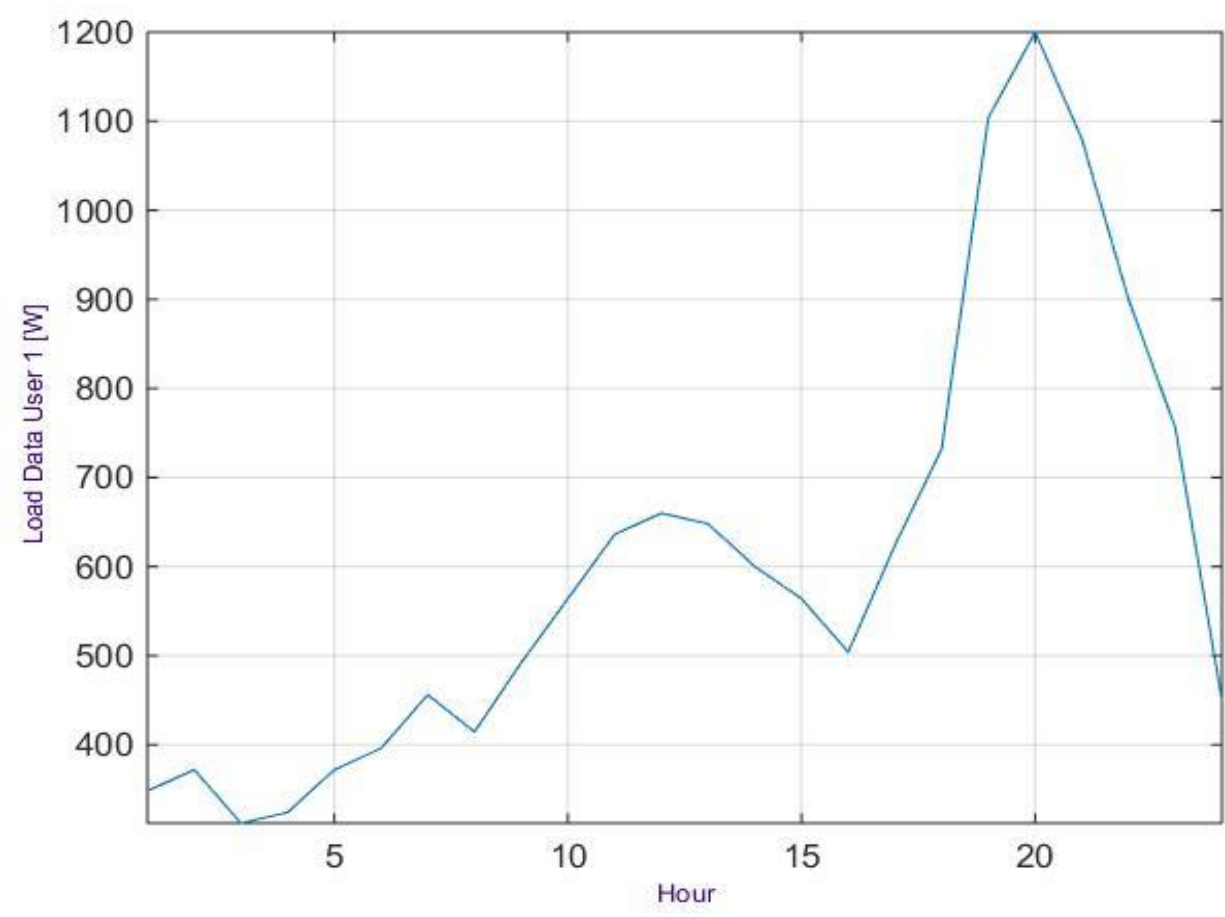

Figure 26. Load demand of user 1. 


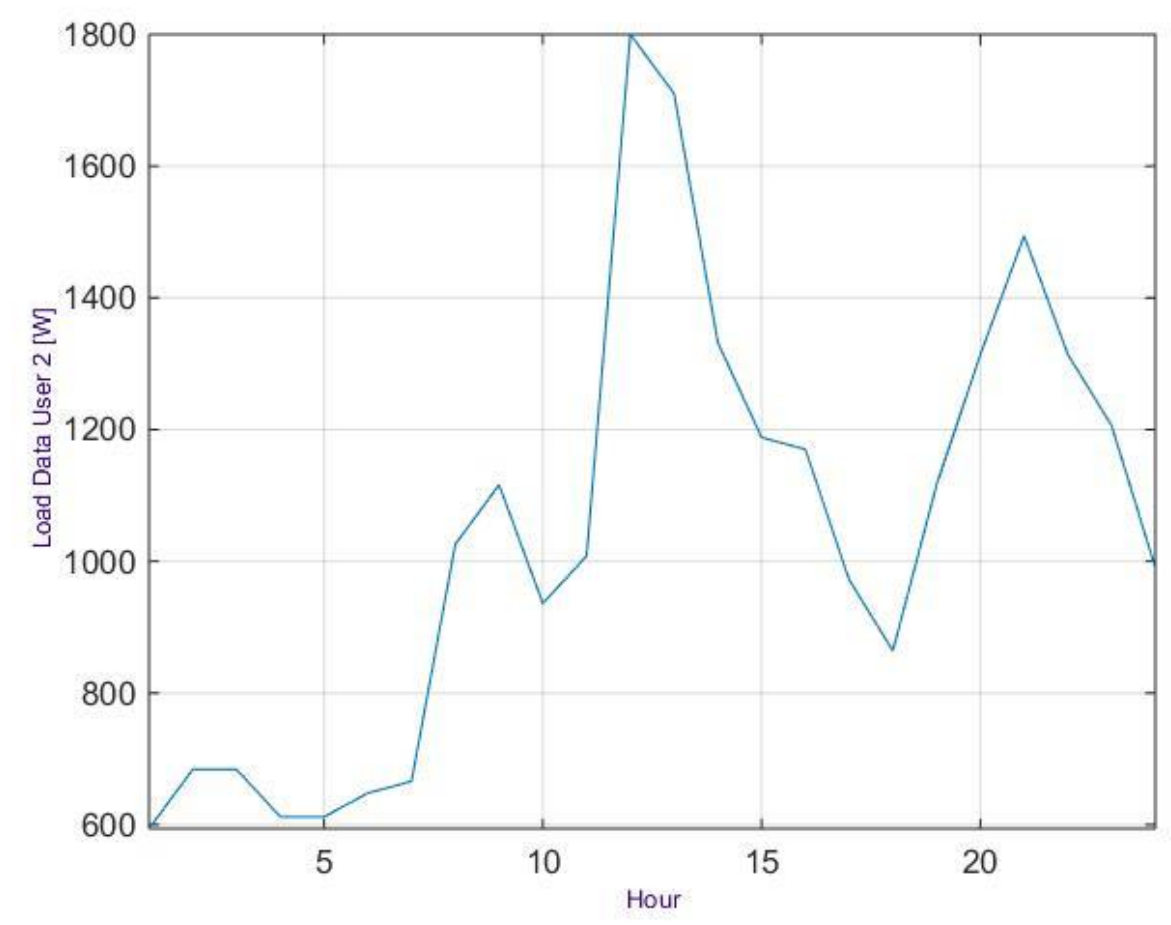

Figure 27. Load demand of User 2.

\section{Constant Factors:}

- Currency: Dollars

- Maximum initial cost: $\$ 15000$

- $\quad$ KWh Cost: $\$ 0.2$

- Solar Area Panel: $72 \mathrm{~m}^{2}$ - 37 panels - 11.8 KW

- Wind turbines maximum number: 4

- Interest rate per year: $4.95 \%$

- Operation and maintenance of solar panels: \$10

- Operation and maintenance of wind turbines: $\$ 30$

- Operation and maintenance of batteries: \$10

- Optimization approach: NPC

\section{Reference and detail of the selected elements:}

Solar panel:

Reference: ASTRONERGY Cristalline CHSM6612P series - 320 Wp NOCT: 46 
Rated power: 320

Short Circuit Current: 9.06 A

Open Circuit Voltage: $45.69 \mathrm{~V}$

Current at maximum power point: $8.93 \mathrm{~A}$

Voltage at maximum power point: $35.86 \mathrm{~V}$

Short circuit current coefficient: $0.05 \% / \mathrm{K}$

Open circuit voltage coefficient: $-0.003 \% / \mathrm{K}$

Panel Dimension: $1.954 \mathrm{~m} * 0.99 \mathrm{~m}$

Price per unit: 236.31

Life cycle: $\$ 25$ years

Wind Turbine:

Reference: Air X

Rated power: $400 \mathrm{~W}$

Power coefficient CP: 0.45

Wind speed cut in: $3.6 \mathrm{~m} / \mathrm{s}$

Wind speed rated power: $12.5 \mathrm{~m} / \mathrm{s}$

Mechanical component efficiency: 1

Wind turbine dimension: $r=1.15 \mathrm{Hub}=10 \mathrm{~m}$

Price per unit: $\$ 849$

Life cycle: 25 years

Battery:

Reference: Energy Cell GH

Maximum current: 167

Maximum voltage: 6

Maximum depth of battery discharge: 10\%

Self discharging rate: $1 \%$

Battery bank efficiency charge: 96\%

Price per unit: 291.25

Life cycle: 12 
Taking into account the above, the possible cases studies to compare both systems are presented in Table 4.

\begin{tabular}{|c|c|c|c|c|}
\hline Case Study & Configuration & Location & User & Reliability [\%] \\
\hline Case1 & Configuration 1 & Miami & User 1 & 50 \\
\hline Case2 & Configuration 1 & Miami & User 1 & 75 \\
\hline Case3 & Configuration 1 & Miami & User 2 & 50 \\
\hline Case4 & Configuration 1 & Miami & User 2 & 75 \\
\hline Case 5 & Configuration 1 & Barranquilla & User 1 & 50 \\
\hline Case6 & Configuration 1 & Barranquilla & User 1 & 75 \\
\hline Case7 & Configuration 1 & Barranquilla & User 2 & 50 \\
\hline Case8 & Configuration 1 & Barranquilla & User 2 & 75 \\
\hline Case9 & Configuration 1 & Fortaleza & User 1 & 50 \\
\hline Case10 & Configuration 1 & Fortaleza & User 1 & 75 \\
\hline Case11 & Configuration 1 & Fortaleza & User 2 & 50 \\
\hline Case12 & Configuration 1 & Fortaleza & User 2 & 75 \\
\hline Case13 & Configuration 2 & Miami & User 1 & 50 \\
\hline Case14 & Configuration 2 & Miami & User 1 & 75 \\
\hline Case15 & Configuration 2 & Miami & User 2 & 50 \\
\hline Case16 & Configuration 2 & Miami & User 2 & 75 \\
\hline Case17 & Configuration 2 & Barranquilla & User 1 & 50 \\
\hline Case18 & Configuration 2 & Barranquilla & User 1 & 75 \\
\hline Case19 & Configuration 2 & Barranquilla & User 2 & 50 \\
\hline Case20 & Configuration 2 & Barranquilla & User 2 & 75 \\
\hline Case21 & Configuration 2 & Fortaleza & User 1 & 50 \\
\hline Case22 & Configuration 2 & Fortaleza & User 1 & 75 \\
\hline Case23 & Configuration 2 & Fortaleza & User 2 & 50 \\
\hline Case24 & Configuration 2 & Fortaleza & User 2 & 75 \\
\hline
\end{tabular}

Table 4. Different cases for the comparison between HYRES and HOMER systems.

The output variables to be compared are:

1. Net Present Value

2. Initial Capital Cost

3. Reliability

4. Number of elements 
Results obtained with HOMER and HYRES for each of the cases of Table 4, are presented in Table 5. In addition to the possible output variables mentioned before, Table 5 shows additional results obtained in HYRES that have proven to be of great utility when designing a hybrid renewable systems such as the payback time and the percentage of hours not supplied with renewable energy (LPS).

\begin{tabular}{|c|c|c|c|c|c|c|c|c|c|c|}
\hline \multirow{3}{*}{ CASE } & \multicolumn{4}{|c|}{ HOMER } & \multicolumn{6}{|c|}{ HYRES } \\
\hline & \multirow{2}{*}{ ICC \$ } & \multirow{2}{*}{ NPC \$ } & \multirow{2}{*}{$\begin{array}{l}\mathrm{RF} \\
\%\end{array}$} & \multirow{2}{*}{$\begin{array}{c}\text { Conf } \\
\text { P-WT-B }\end{array}$} & \multirow{2}{*}{ ICC \$ } & \multirow{2}{*}{ NPC \$ } & \multirow{2}{*}{$\begin{array}{l}\text { RF } \\
\%\end{array}$} & \multirow{2}{*}{$\begin{array}{c}\text { Conf } \\
\text { P-WT-B }\end{array}$} & \multirow{2}{*}{ PY } & \multirow{2}{*}{ LPS \% } \\
\hline & & & & & & & & & & \\
\hline Case1 & 2507 & 11254 & 50 & $2.56-0-3$ & 2236 & 11720 & 51 & $8-0-3$ & 7 & 62.5 \\
\hline Case2 & 4774 & 11409 & 76 & $3,31-0-8$ & 4583 & 10916 & 77 & $12-0-6$ & 6 & 37.5 \\
\hline Case3 & 3810 & 17726 & 52 & $3.98-0-3$ & 3560 & 18342 & 50 & $14-0-1$ & 7 & 62.5 \\
\hline Case4 & 7359 & 17918 & 76 & 5.63-0- & 7347 & 17946 & 77 & $20-0-9$ & 6 & 42 \\
\hline Case5 & 4739 & 8577 & 98 & $2.46-0-10$ & 4858 & 8541 & 100 & $7-0-11$ & 8 & 0 \\
\hline Case6 & 4739 & 8577 & 98 & $2.46-0-10$ & 4858 & 8541 & 100 & 7-0-11 & 8 & 0 \\
\hline Case7 & 7170 & 12980 & 97 & $4.19-0-14$ & 6968 & 12570 & 98 & $11-0-15$ & 7 & 4 \\
\hline Case8 & 7170 & 12980 & 97 & $4.19-0-14$ & 6968 & 12570 & 98 & $11-0-15$ & 7 & 4 \\
\hline Case9 & 4124 & 7796 & 93 & $1.31-2-5$ & 3099 & 6166 & 100 & $1-2-4$ & 5 & 0 \\
\hline Case 10 & 4124 & 7796 & 93 & $1.31-2-5$ & 3099 & 6166 & 100 & $1-2-4$ & 5 & 0 \\
\hline Case11 & 5175 & 19358 & 53 & $3.16-3-1$ & 5088 & 10261 & 100 & $1-4-5$ & 5 & 0 \\
\hline Case12 & 9587 & 20771 & 75 & $5.62-4-7$ & 5088 & 10261 & 100 & $1-4-5$ & 5 & 0 \\
\hline Case13 & 8746 & -29341 & 87 & $8.75-0-0$ & 8743 & -15728 & 85 & $37-0-0$ & 3 & 58.3 \\
\hline Case14 & 8746 & -29341 & 87 & $11.8-0-0$ & 8743 & -15728 & 85 & $37-0-0$ & 3 & 58.3 \\
\hline Case15 & 8746 & -18433 & 81 & $11.8-0-0$ & 8743 & -12322 & 78.7 & $37-0-0$ & 4 & 54.1 \\
\hline Case 16 & 8746 & -18433 & 81 & $11.8-0-0$ & 8743 & -12322 & 78.7 & $37-0-0$ & 4 & 54.1 \\
\hline Case17 & 8746 & -47532 & 90 & $11.8-0-0$ & 8743 & -65279 & 92.5 & $37-0-0$ & 4 & 58.3 \\
\hline Case 18 & 8746 & -47532 & 90 & $11.8-0-0$ & 8743 & -65279 & 92.5 & $37-0-0$ & 4 & 58.3 \\
\hline Case19 & 8746 & -36623 & 86 & $11.8-0-0$ & 8743 & -50241 & 87 & $37-0-0$ & 4 & 58.3 \\
\hline Case20 & 8746 & -36623 & 86 & $11.8-0-0$ & 8743 & -50241 & 87 & $37-0-0$ & 4 & 58.3 \\
\hline Case21 & 12142 & -34482 & 100 & $11.8-4-0$ & 12139 & -52282 & 100 & $37-4-0$ & 4 & 8.3 \\
\hline Case22 & 12142 & -34482 & 100 & $11.8-4-0$ & 12139 & -52282 & 100 & $37-4-0$ & 4 & 8.3 \\
\hline Case 23 & 12142 & -23573 & 92 & $11.8-4-0$ & 12139 & -40867 & 94 & $37-4-0$ & 4 & 8.3 \\
\hline Case24 & 12142 & -23573 & 92 & $11.8-4-0$ & 12139 & -40867 & 94 & $37-4-0$ & 4 & 8.3 \\
\hline
\end{tabular}

Table 5. Comparison between HOMER and HYRES systems.

Below, Figures 28 and 29 shows the behavior in terms of power of the systems during a day in a case example of the ones presented in Table 5. 


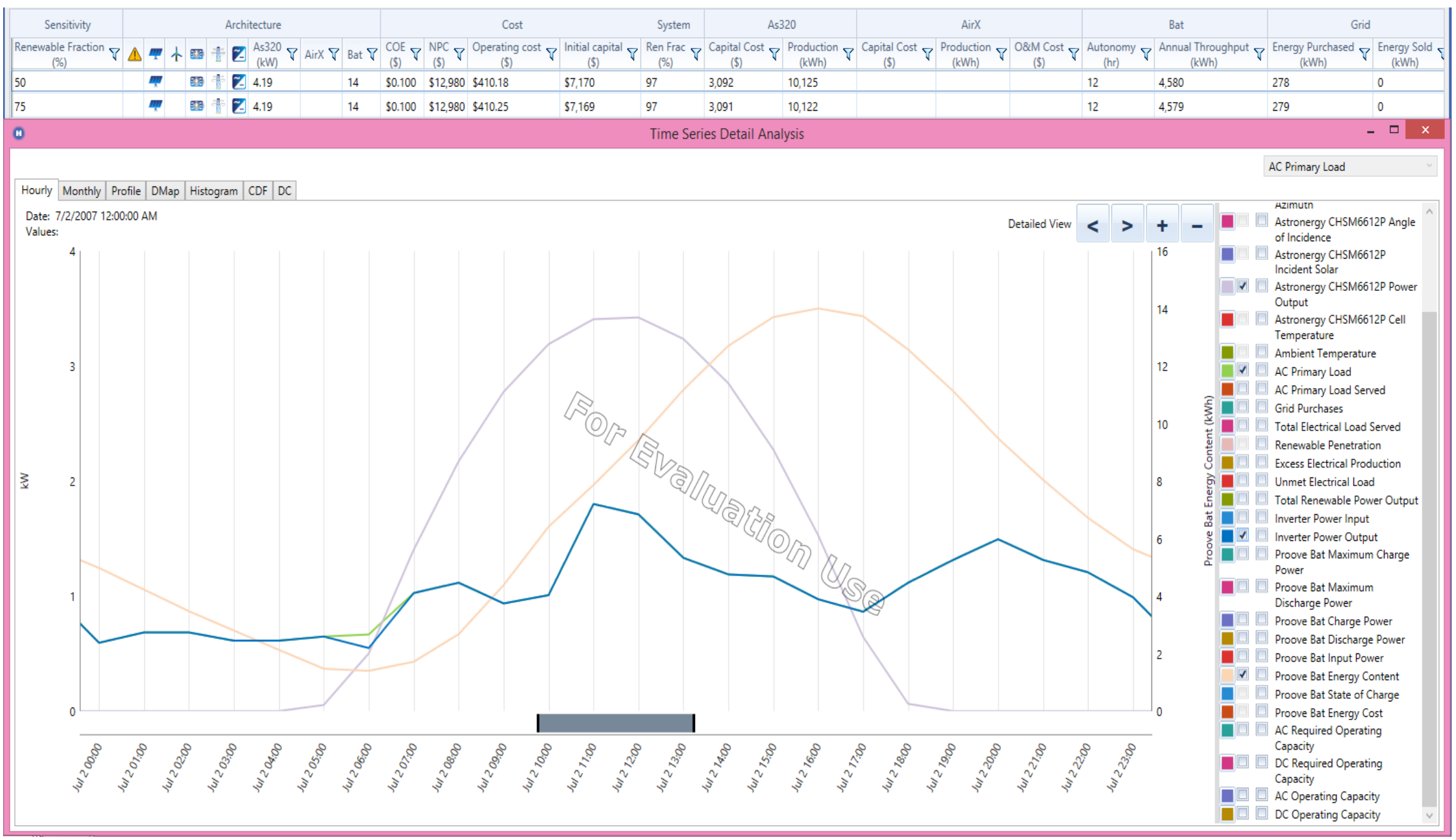

Figure 28. Result from HOMER for Case7. 


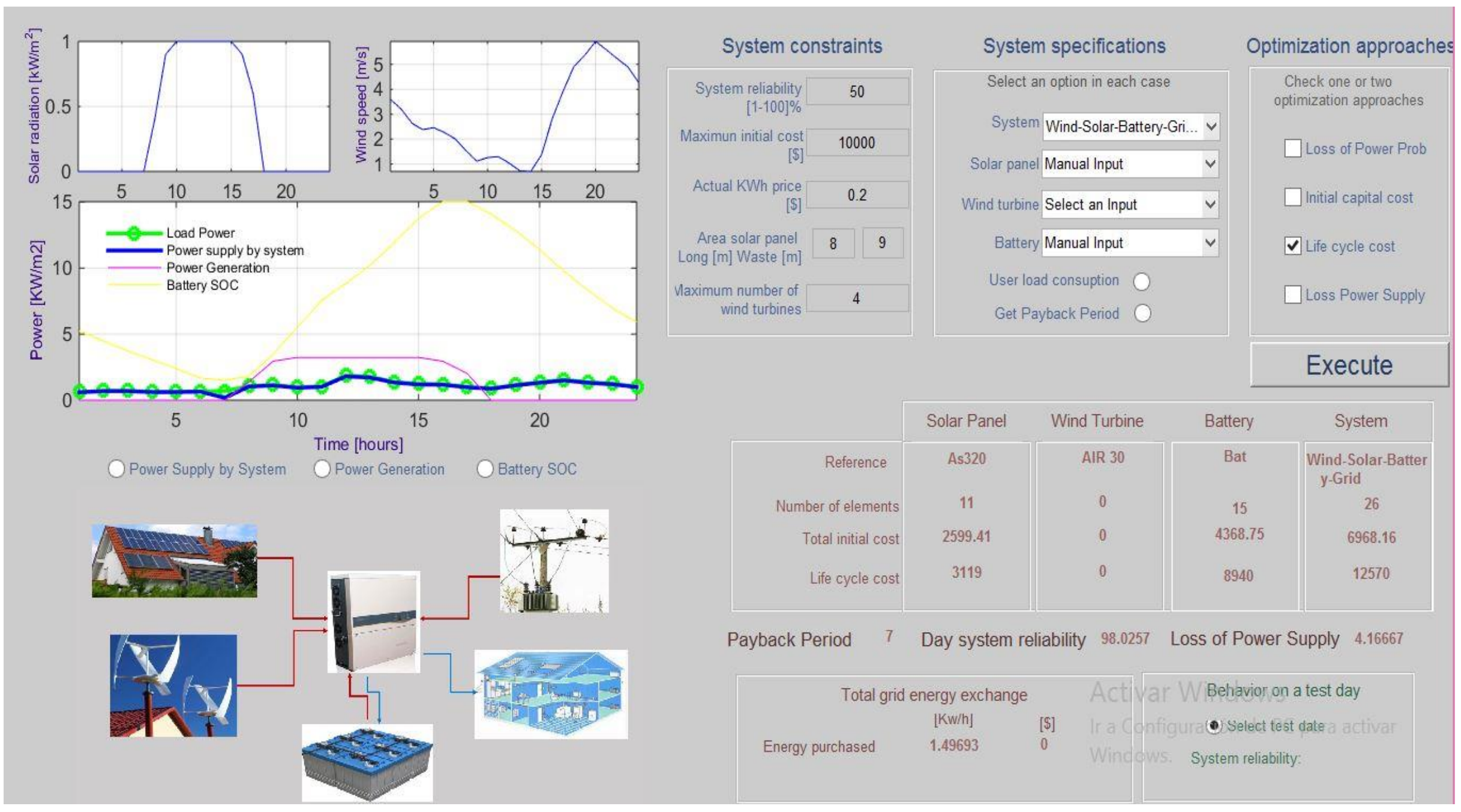

Figure 29. Result from HYRES for Case7. 
Table 5 shows orange underlined rows, these are cases with equal variant factors except the required reliability, which changes from $50 \%$ to $75 \%$ in each case. When making the comparison between both systems for this change of reliability, it can be observed that there are many similarities in the answers, as in the ICC that changes from case one to case two in a ratio of 1.9 and 2.0 for HOMER and HYRES respectively. However, there is a change in the NPC, in HOMER software increases from case 1 to case 2, in HYRES decreases.

As for the rows underlined in green, there is a change in the city where the system is installed. The first city is Barranquilla, it has good values of solar radiation and low values for wind speed, on the other hand, Fortaleza is a city with high speed values of wind and average values of solar radiation. In the results it is observed that both systems are adjusted to these climatic conditions, prioritizing in case of Fortaleza wind energy and in the case of Barranquilla solar energy.

The underlined rows in blue have a change in the demand of energy in the users, besides the type of system selected and by the possibility of sale of energy to the network, both systems maximize the use of renewable energies looking for obtain the highest profitability in the sale of energy. The notorious change in this system is that it is not possible to deliver the same amount of renewable energy in both loads, mainly due to the demand in hours where there is no generation and the absence of a storage system.

Finally, for rows underlined with red, there is a change in the configuration. The results show that although in the second configuration the renewable generation seeks its limit to have benefits from the sale of energy to the grid, in the first one is limited the use of renewable energy to meet user requirements.

Next, a comparison of the results obtained in the HYRES system implemented in this thesis by varying the different input possibilities is shown. The parameters established to make the comparison were: 


\section{Variable parameters:}

System reliability: $50 \%-75 \%$

Initial cost: 8000-16000

System implemented: 4

Objective optimization approach: 4

Multiobjective optimization approach: 4

\section{Constant parameters:}

Currency: Dollar

Location: Barranquilla, Colombia

Cost of KWh: 0.2

Available area: $8 m * 9 m$

References of elements: suggested by the system

User request: User 2

Interest rate: $5 \%$

Cost of operation and maintenance panels: 10

Cost of operation and maintenance wind turbines: 70

Cost of operation and maintenance batteries: 12

Tables 6 and 7 shows the results obtained for the different cases. Table 6 shows the results by varying the optimization approaches in a mono-objective optimization. Table 7 shows the results by varying the optimization approaches in a multiobjective optimization. 


\begin{tabular}{|c|c|c|c|c|c|c|c|c|c|}
\hline \multirow{2}{*}{ CASE } & \multicolumn{4}{|c|}{ PARAMETERS } & \multicolumn{5}{|c|}{ RESULTS } \\
\hline & $\begin{array}{c}\text { Reliabili } \\
\text { ty } \%\end{array}$ & $\begin{array}{l}\text { Initial } \\
\text { Cost \$ }\end{array}$ & $\begin{array}{l}\text { Configu } \\
\text {-ration }\end{array}$ & Approach & ICC \$ & LCC \$ & $\begin{array}{c}\text { Payback } \\
\text { years }\end{array}$ & $\begin{array}{c}\text { Reliabili } \\
\text { ty } \%\end{array}$ & LPS \% \\
\hline 1 & 50 & 8000 & 1 & LPP & 7270 & 20012 & 9 & 100 & 0 \\
\hline 2 & 50 & 8000 & 1 & ICC & 1478 & 3221 & 3 & 51.2 & 58.3 \\
\hline 3 & 50 & 8000 & 1 & LCC & 1478 & 3221 & 3 & 51.2 & 58.3 \\
\hline 4 & 50 & 8000 & 1 & LPSP & 7187 & 15619 & 8 & 100 & 0 \\
\hline 5 & 50 & 8000 & 2 & LPP & 7920 & 13558 & No & 87.086 & 25 \\
\hline 6 & 50 & 8000 & 2 & ICC & 2235 & 4933 & 4 & 52.2 & 66.7 \\
\hline 7 & 50 & 8000 & 2 & LCC & 2520 & 3930 & 5 & 52.6 & 100 \\
\hline 8 & 50 & 8000 & 2 & LPSP & 7902 & 12271 & 8 & 86.42 & 25 \\
\hline 9 & 50 & 8000 & 3 & LPP & 18253 & 33433 & No & 100 & 0 \\
\hline 10 & 50 & 8000 & 3 & ICC & 4576 & 12511 & 6 & 100 & 0 \\
\hline 11 & 50 & 8000 & 3 & LCC & 5296 & 10756 & 6 & 100 & 0 \\
\hline 12 & 50 & 8000 & 3 & LPSP & 13906 & 26843 & 18 & 100 & 0 \\
\hline 13 & 50 & 8000 & 4 & LPP & 7920 & 13558 & 8 & 87.1 & 25 \\
\hline 14 & 50 & 8000 & 4 & ICC & 2235 & 4933 & 4 & 52.2 & 66.7 \\
\hline 15 & 50 & 8000 & 4 & LCC & 2520 & 3930 & 5 & 52.6 & 100 \\
\hline 16 & 50 & 8000 & 4 & LPSP & 7653 & 12445 & 8 & 86.4 & 25 \\
\hline 17 & 50 & 16000 & 1 & LPP & 11780 & 26033 & 14 & 100 & 0 \\
\hline 18 & 50 & 16000 & 1 & ICC & 1478 & 3221 & 3 & 51.82 & 58.33 \\
\hline 19 & 50 & 16000 & 1 & LCC & 1478 & 3221 & 3 & 51.8 & 58.3 \\
\hline 20 & 50 & 16000 & 1 & LPSP & 10938 & 24758 & 13 & 100 & 0 \\
\hline 21 & 50 & 16000 & 2 & LPP & 12436 & 19088 & 11 & 87.1 & 25 \\
\hline 22 & 50 & 16000 & 2 & ICC & 2235 & 4933 & 4 & 52.2 & 66.7 \\
\hline 23 & 50 & 16000 & 2 & LCC & 2520 & 3930 & 5 & 52.6 & 100 \\
\hline 24 & 50 & 16000 & 2 & LPSP & 14672 & 28829 & No & 84.4 & 25 \\
\hline 25 & 50 & 16000 & 3 & LPP & 11206 & 24346 & 18 & 100 & 0 \\
\hline 26 & 50 & 16000 & 3 & ICC & 4576 & 12511 & 6 & 100 & 0 \\
\hline 27 & 50 & 16000 & 3 & LCC & 5296 & 10756 & 6 & 100 & 0 \\
\hline 28 & 50 & 16000 & 3 & LPSP & 9675 & 22773 & 14 & 100 & 0 \\
\hline 29 & 50 & 16000 & 4 & LPP & 6000 & 9946 & 10 & 43.9 & 62.5 \\
\hline 30 & 50 & 16000 & 4 & ICC & 2235 & 4933 & 4 & 52.2 & 66.7 \\
\hline 31 & 50 & 16000 & 4 & LCC & 2520 & 3930 & 5 & 52.6 & 100 \\
\hline 32 & 50 & 16000 & 4 & LPSP & 6000 & 9946 & 10 & 43.88 & 62.5 \\
\hline 33 & 75 & 8000 & 1 & LPP & 7760 & 19476 & 7 & 100 & 0 \\
\hline 34 & 75 & 8000 & 1 & ICC & 5232 & 12769 & 4 & 79.8 & 33.3 \\
\hline 35 & 75 & 8000 & 1 & LCC & 5232 & 12769 & 4 & 79.8 & 33.3 \\
\hline 36 & 75 & 8000 & 1 & LPSP & 7832 & 20099 & 7 & 100 & 0 \\
\hline 37 & 75 & 8000 & 2 & LPP & 8020 & 14080 & 10 & 43.2 & 70.1 \\
\hline
\end{tabular}




\begin{tabular}{|c|c|c|c|c|c|c|c|c|c|}
\hline 38 & 75 & 8000 & 2 & ICC & 8020 & 14080 & 10 & 43.2 & 70.1 \\
\hline 39 & 75 & 8000 & 2 & LCC & 8020 & 14080 & 10 & 43.2 & 70.1 \\
\hline 40 & 75 & 8000 & 2 & LPSP & 17346 & 34414 & 14 & 100 & 0 \\
\hline 41 & 75 & 8000 & 3 & LPP & 13327 & 27607 & 9 & 100 & 0 \\
\hline 42 & 75 & 8000 & 3 & ICC & 7600 & 19175 & 7 & 100 & 0 \\
\hline 43 & 75 & 8000 & 3 & LCC & 7600 & 19175 & 7 & 100 & 0 \\
\hline 44 & 75 & 8000 & 3 & LPSP & 19823 & 35754 & 14 & 100 & 0 \\
\hline 45 & 75 & 8000 & 4 & LPP & 8020 & 14080 & 10 & 43.3 & 70.8 \\
\hline 46 & 75 & 8000 & 4 & ICC & 8020 & 14080 & 10 & 43.3 & 70.8 \\
\hline 47 & 75 & 8000 & 4 & LCC & 8020 & 14080 & 10 & 43.3 & 70.8 \\
\hline 48 & 75 & 8000 & 4 & LPSP & 8020 & 14080 & 10 & 43.3 & 70.8 \\
\hline 49 & 75 & 16000 & 1 & LPP & 14520 & 33270 & 14 & 100 & 0 \\
\hline 50 & 75 & 16000 & 1 & ICC & 5232 & 12769 & 4 & 79.8 & 33.3 \\
\hline 51 & 75 & 16000 & 1 & LCC & 5232 & 12769 & 4 & 79.8 & 33.3 \\
\hline 52 & 75 & 16000 & 1 & LPSP & 12024 & 27802 & 9 & 100 & 0 \\
\hline 53 & 75 & 16000 & 2 & LPP & 15944 & 25669 & 15 & 47.2 & 70.8 \\
\hline 54 & 75 & 16000 & 2 & ICC & 6000 & 9946 & 22 & 6.42 & 100 \\
\hline 55 & 75 & 16000 & 2 & LCC & 15944 & 25669 & 15 & 47.2 & 70.8 \\
\hline 56 & 75 & 16000 & 2 & LPSP & 15865 & 23899 & 15 & 46.4 & 70.8 \\
\hline 57 & 75 & 16000 & 3 & LPP & 21785 & 37929 & 17 & 100 & 0 \\
\hline 58 & 75 & 16000 & 3 & ICC & 7600 & 19175 & 7 & 100 & 0 \\
\hline 59 & 75 & 16000 & 3 & LCC & 7600 & 19175 & 7 & 100 & 0 \\
\hline 60 & 75 & 16000 & 3 & LPSP & 25580 & 43927 & 19 & 100 & 0 \\
\hline 61 & 75 & 16000 & 4 & LPP & 15865 & 23899 & 15 & 46.4 & 70.8 \\
\hline 62 & 75 & 16000 & 4 & ICC & 15944 & 25669 & 15 & 47.2 & 70.8 \\
\hline 63 & 75 & 16000 & 4 & LCC & 15944 & 25669 & 15 & 47.2 & 70.8 \\
\hline 64 & 75 & 16000 & 4 & LPSP & 15944 & 25669 & 15 & 47.2 & 70.8 \\
\hline
\end{tabular}

Table 6. Results of HYRES with mono-objective optimization 


\begin{tabular}{|c|c|c|c|c|c|c|c|c|c|}
\hline \multirow{2}{*}{ CASE } & \multicolumn{4}{|c|}{ PARAMETERS } & \multicolumn{5}{|c|}{ RESULTS } \\
\hline & $\begin{array}{c}\text { Reliabili } \\
\text { ty } \%\end{array}$ & $\begin{array}{l}\text { Initial } \\
\text { Cost \$ }\end{array}$ & $\begin{array}{l}\text { Configu } \\
\text {-ration }\end{array}$ & Approach & ICC \$ & LCC \$ & $\begin{array}{c}\text { Payback } \\
\text { years }\end{array}$ & $\begin{array}{c}\text { Reliabili } \\
\text { ty } \%\end{array}$ & LPS \% \\
\hline 1 & 50 & 8000 & 1 & LPP-ICC & 4508 & 10096 & 6 & 98.1 & 4.16 \\
\hline 2 & 50 & 8000 & 1 & LPP-LCC & 5296 & 10756 & 6 & 100 & 0 \\
\hline 3 & 50 & 8000 & 1 & LPSP-ICC & 4576 & 12511 & 6 & 100 & 0 \\
\hline 4 & 50 & 8000 & 1 & LPSP-LCC & 5296 & 10756 & 6 & 100 & 0 \\
\hline 5 & 50 & 8000 & 2 & LPP-ICC & 7920 & 13558 & 8 & 87.2 & 25 \\
\hline 6 & 50 & 8000 & 2 & LPP-LCC & 7920 & 13558 & 8 & 87.1 & 25 \\
\hline 7 & 50 & 8000 & 2 & LPSP-ICC & 6420 & 11071 & 7 & 86.4 & 25 \\
\hline 8 & 50 & 8000 & 2 & LPSP-LCC & 6420 & 11071 & 7 & 86.4 & 25 \\
\hline 9 & 50 & 8000 & 3 & LPP-ICC & 4576 & 12511 & 6 & 100 & 0 \\
\hline 10 & 50 & 8000 & 3 & LPP-LCC & 4740 & 11021 & 6 & 100 & 0 \\
\hline 11 & 50 & 8000 & 3 & LPSP-ICC & 4576 & 12511 & 6 & 100 & 0 \\
\hline 12 & 50 & 8000 & 3 & LPSP-LCC & 4740 & 11021 & 6 & 100 & 0 \\
\hline 13 & 50 & 8000 & 4 & LPP-ICC & 7920 & 13558 & 8 & 87.1 & 25 \\
\hline 14 & 50 & 8000 & 4 & LPP-LCC & 7920 & 13558 & 8 & 87.1 & 25 \\
\hline 15 & 50 & 8000 & 4 & LPSP-ICC & 6420 & 11071 & 7 & 86.4 & 25 \\
\hline 16 & 50 & 8000 & 4 & LPSP-LCC & 6420 & 11071 & 7 & 86.4 & 25 \\
\hline 17 & 50 & 16000 & 1 & LPP-ICC & 4508 & 10096 & 6 & 98.1 & 4.16 \\
\hline 18 & 50 & 16000 & 1 & LPP-LCC & 5296 & 10756 & 6 & 100 & 0 \\
\hline 19 & 50 & 16000 & 1 & LPSP-ICC & 4576 & 12511 & 6 & 100 & 0 \\
\hline 20 & 50 & 16000 & 1 & LPSP-LCC & 5296 & 10756 & 6 & 100 & 0 \\
\hline 21 & 50 & 16000 & 2 & LPP-ICC & 7920 & 13558 & 8 & 87.2 & 25 \\
\hline 22 & 50 & 16000 & 2 & LPP-LCC & 7920 & 13558 & 8 & 87.1 & 25 \\
\hline 23 & 50 & 16000 & 2 & LPSP-ICC & 6420 & 11071 & 7 & 86.4 & 25 \\
\hline 24 & 50 & 16000 & 2 & LPSP-LCC & 6420 & 11071 & 7 & 86.4 & 25 \\
\hline 25 & 50 & 16000 & 3 & LPP-ICC & 4576 & 12511 & 6 & 100 & 0 \\
\hline 26 & 50 & 16000 & 3 & LPP-LCC & 4740 & 11021 & 6 & 100 & 0 \\
\hline 27 & 50 & 16000 & 3 & LPSP-ICC & 7920 & 13558 & 8 & 87.1 & 25 \\
\hline 28 & 50 & 16000 & 3 & LPSP-LCC & 7920 & 13558 & 8 & 87.1 & 25 \\
\hline 29 & 50 & 16000 & 4 & LPP-ICC & 7920 & 13558 & 8 & 87.1 & 25 \\
\hline 30 & 50 & 16000 & 4 & LPP-LCC & 7920 & 13558 & 8 & 87.1 & 25 \\
\hline 31 & 50 & 16000 & 4 & LPSP-ICC & 6420 & 11071 & 7 & 86.4 & 25 \\
\hline 32 & 50 & 16000 & 4 & LPSP-LCC & 6420 & 11071 & 7 & 86.4 & 25 \\
\hline 33 & 75 & 8000 & 1 & LPP-ICC & 7528 & 18551 & 6 & 100 & 0 \\
\hline 34 & 75 & 8000 & 1 & LPP-LCC & 7848 & 19153 & 7 & 100 & 0 \\
\hline 35 & 75 & 8000 & 1 & LPSP-ICC & 7600 & 19175 & 7 & 100 & 0 \\
\hline 36 & 75 & 8000 & 1 & LPSP-LCC & 7600 & 19175 & 7 & 100 & 0 \\
\hline 37 & 75 & 8000 & 2 & LPP-ICC & 2080 & 3912 & 4 & 36.5 & 75 \\
\hline
\end{tabular}




\begin{tabular}{|c|c|c|c|c|c|c|c|c|c|}
\hline 38 & 75 & 8000 & 2 & LPP-LCC & 2080 & 3912 & 4 & 36.5 & 75 \\
\hline 39 & 75 & 8000 & 2 & LPSP-ICC & 2080 & 3912 & 4 & 36.5 & 75 \\
\hline 40 & 75 & 8000 & 2 & LPSP-LCC & 2080 & 3912 & 4 & 36.5 & 75 \\
\hline 41 & 75 & 8000 & 3 & LPP-ICC & 7600 & 19175 & 7 & 100 & 0 \\
\hline 42 & 75 & 8000 & 3 & LPP-LCC & 7600 & 19175 & 7 & 100 & 0 \\
\hline 43 & 75 & 8000 & 3 & LPSP-ICC & 7600 & 19175 & 7 & 100 & 0 \\
\hline 44 & 75 & 8000 & 3 & LPSP-LCC & 7600 & 19175 & 7 & 100 & 0 \\
\hline 45 & 75 & 8000 & 4 & LPP-ICC & 2080 & 3912 & 4 & 36.5 & 75 \\
\hline 46 & 75 & 8000 & 4 & LPP-LCC & 2080 & 3912 & 4 & 36.5 & 75 \\
\hline 47 & 75 & 8000 & 4 & LPSP-ICC & 2080 & 3912 & 4 & 36.5 & 75 \\
\hline 48 & 75 & 8000 & 4 & LPSP-LCC & 2080 & 3912 & 4 & 36.5 & 75 \\
\hline 49 & 75 & 16000 & 1 & LPP-ICC & 7528 & 18551 & 6 & 100 & 0 \\
\hline 50 & 75 & 16000 & 1 & LPP-LCC & 7848 & 19153 & 7 & 100 & 0 \\
\hline 51 & 75 & 16000 & 1 & LPSP-ICC & 7600 & 19175 & 7 & 100 & 0 \\
\hline 52 & 75 & 16000 & 1 & LPSP-LCC & 7600 & 19175 & 7 & 100 & 0 \\
\hline 53 & 75 & 16000 & 2 & LPP-ICC & 2080 & 3912 & 4 & 36.5 & 75 \\
\hline 54 & 75 & 16000 & 2 & LPP-LCC & 2080 & 3912 & 4 & 36.5 & 75 \\
\hline 55 & 75 & 16000 & 2 & LPSP-ICC & 2080 & 3912 & 4 & 36.5 & 75 \\
\hline 56 & 75 & 16000 & 2 & LPSP-LCC & 2080 & 3912 & 4 & 36.5 & 75 \\
\hline 57 & 75 & 16000 & 3 & LPP-ICC & 7600 & 19175 & 7 & 100 & 0 \\
\hline 58 & 75 & 16000 & 3 & LPP-LCC & 7600 & 19175 & 7 & 100 & 0 \\
\hline 59 & 75 & 16000 & 3 & LPSP-ICC & 7600 & 19175 & 7 & 100 & 0 \\
\hline 60 & 75 & 16000 & 3 & LPSP-LCC & 7600 & 19175 & 7 & 100 & 0 \\
\hline 61 & 75 & 16000 & 4 & LPP-ICC & 2080 & 3912 & 4 & 36.5 & 75 \\
\hline 62 & 75 & 16000 & 4 & LPP-LCC & 2080 & 3912 & 4 & 36.5 & 75 \\
\hline 63 & 75 & 16000 & 4 & LPSP-ICC & 2080 & 3912 & 4 & 36.5 & 75 \\
\hline 64 & 75 & 16000 & 4 & LPSP-LCC & 2080 & 3912 & 4 & 36.5 & 75 \\
\hline
\end{tabular}

Table 7. Results of HYRES with multi-objective optimization

Tables 6 and 7 show underlined rows with different colors. The rows with orange color show equal variance factors except for the optimization approach, which in table 6 is mono-objective and in table 7 is multi-objective. For the first case, it is observed that in reliability LPP and LPSP optimization approaches, 100\% system reliability is obtained by paying a high cost for this, however, when referring to ICC and LCC cost approaches, cost decreases significantly but low reliability values are obtained. It needs to be highlighted that the cases in which the investment is recovered faster is in the lower cost. On the other hand, as regards Table 7 , it is 
observed that the results are similar in each case studied, reducing initial costs and maximizing reliability.

As for the rows underlined with blue, they change in the configuration used. According to this, the reliability obtained is maximized, although, in cases where the storage system is not available, it is smaller.

The purple rows reflect a change in the minimum required reliability. For monoobjective, minimum reliability values have been found at low initial cost levels compared to Table 7, which increases reliability values in both cases.

The rows underlined with green color refer to the maximum value of initial cost, in this case, for mono-objective is limited to cover at the lowest cost the required reliability, for multi-objective, the reliability increases taking into account the established constraint.

\subsection{Analysis of Results}

When comparing the data obtained with the HOMER system and with the system presented in this thesis HYRES, we can perform a statistical analysis between the data obtained by each response variable in order to look at the relationship between them. For this, a variance analysis was performed first to compare the means of the results, followed by a multivariate test. These processes were performed using the anovan and multcompare toolbox of MATLAB.

Analysis of variance compares the means of several groups to test the hypothesis that they are all equal, against the general alternative that they are not all equal. After the analysis of variance a test that allows identifying which means are different and furthermore carry out a comparison between a means group is necessary to implement. In this proposal was used multiple comparison test named TukeyKramer to identify the means significantly different. When there are many groups, there are many pairs to compare. If an ordinary t-test is applied in this situation, the 
alpha value would apply to each comparison, so the chance of incorrectly finding a significant difference would increase with the number of comparisons. Multiple comparison procedures are designed to provide an upper bound on the probability that any comparison will be incorrectly found significant.

\section{INITIAL CAPITAL COST (ICC) comparison between HOMER and HYRES:}

Figure 30 shows the results of the multiple comparison test of the Initial Capital Cost of HOMER and HYRES system. Results shows that there are no means significantly different from between the systems experienced.

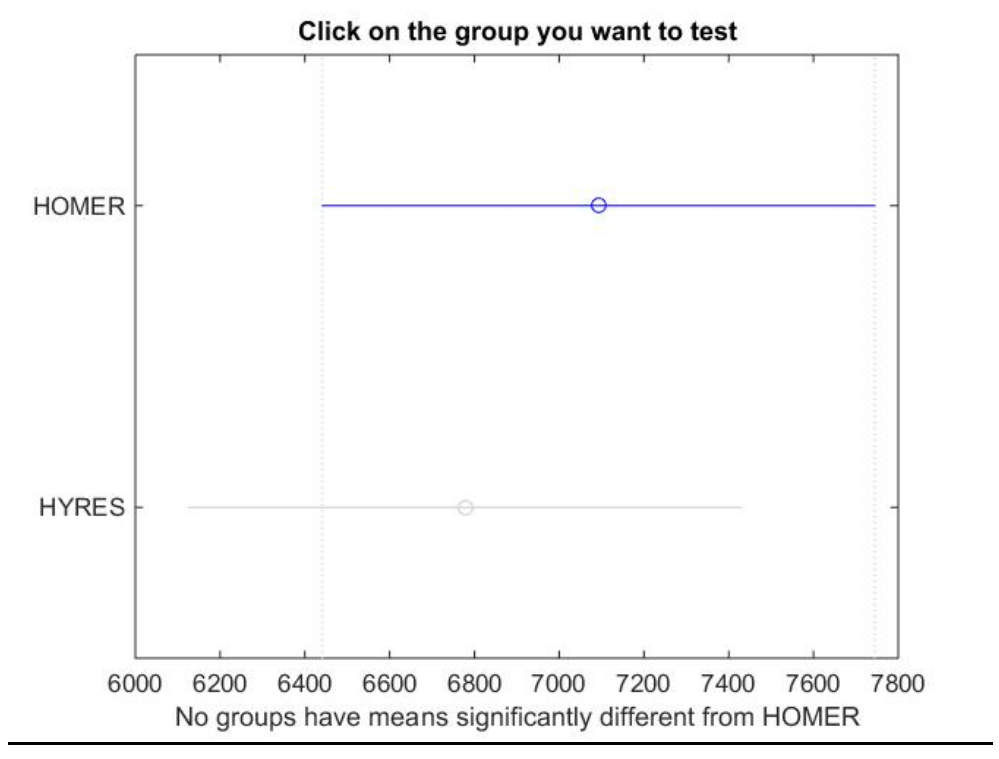

Figure 30. Multiple comparison test of ICC.

\section{NET PRESENT VALUE (NPV) comparison between HOMER and HYRES:}

Figure 31 shows the results of the multiple comparison test of the Net Present Value of HOMER and HYRES system. Results shows that there are no means significantly different from between the systems experienced. 


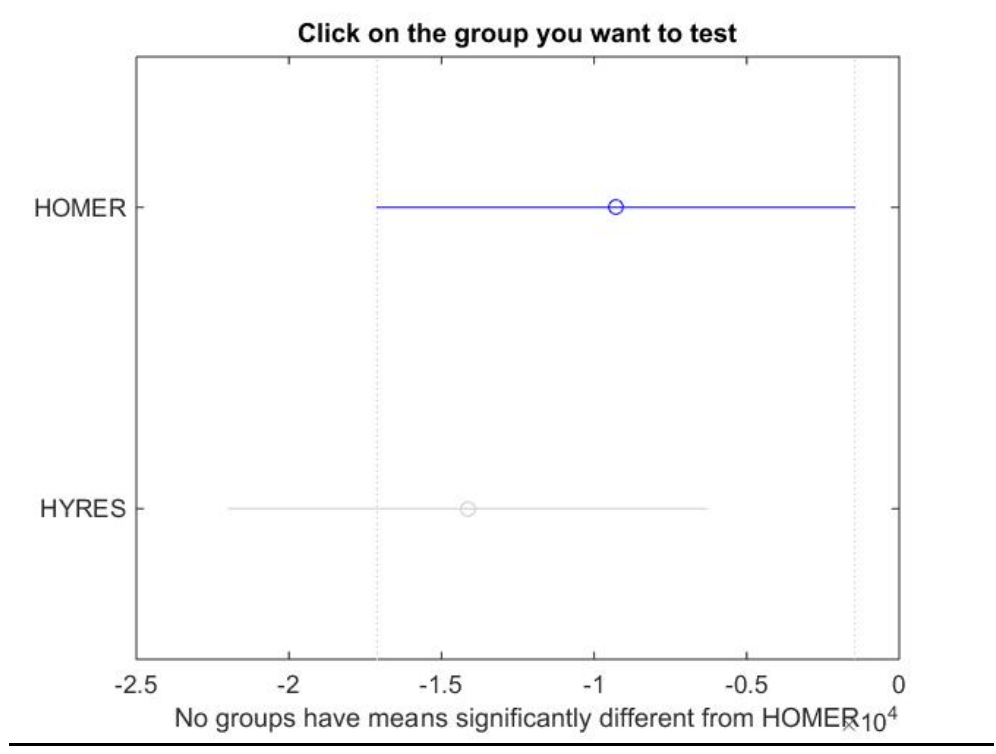

Figure 31. Multiple comparison test of NPC.

\section{RENEWABLE FRACTION (RF) comparison between HOMER and HYRES:}

Figure 32 shows the results of the multiple comparison test of the Renewable Fraction of HOMER and HYRES system. Results shows that there are no means significantly different from between the systems experienced.

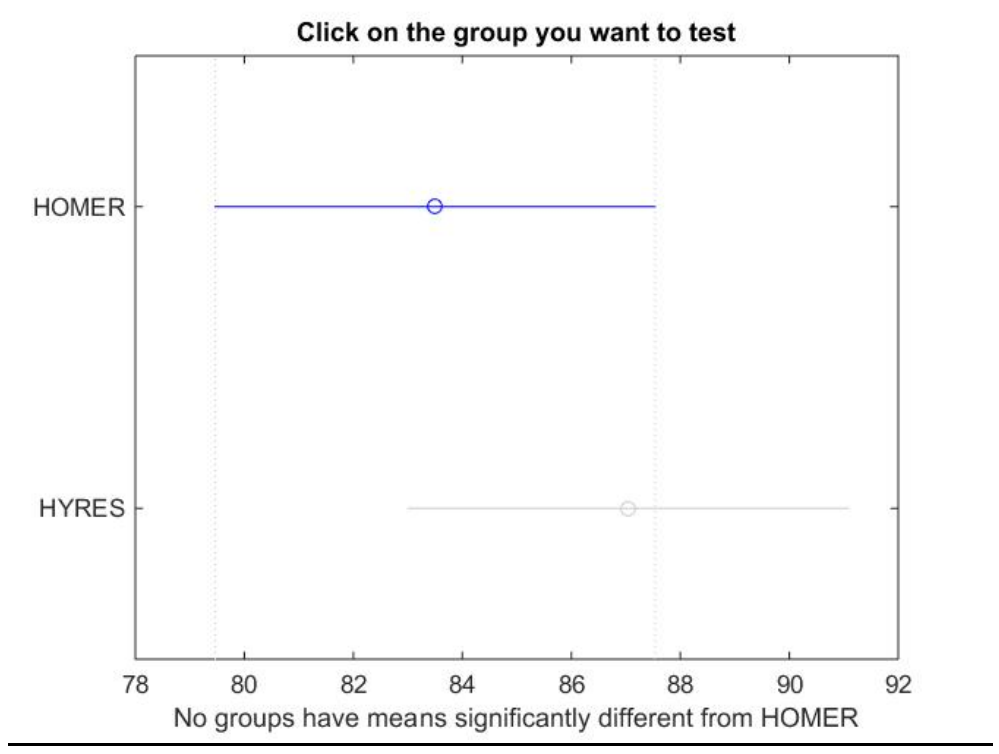

Figure 32. Multiple comparison test of Renewable Fraction.

According to the previous results, it is possible to observe that there is no statistical difference between the HOMER responses means values and the mean responses values of the HYRES. With this, it is possible to verify the reliability of the implemented system as compared to a commercial system that is the leader 
software in modeling microgrids worldwide. However, HOMER system only provides the possibility of performing the optimization under parameters of Net Present Cost (NPC), in addition of having no initial cost restrictions. Another significant difference between the systems compared is that the number of solar panels in HOMER is not an integer value and the optimization is done according to areas and powers parameters, while HYRES performs the optimization with the number of solar panels to be used. This is done taking into account the area parameters of the solar panel reference selected.

Next, a comparison between the results obtained in the system implemented in this thesis HYRES according to the optimization approaches is presented. Figure 33, shows the multiple comparison test of the mono-objective and multi-objective optimization experiments.

According to figure 33, it is possible to observe that in cases associated with costs, for example ICC and LCC, the multi-objective approach presents a statistically mean significantly lower than the mono-objective approach. As for the efficiency case, it is shown that the mean is not significantly different, however, the power loss supply LPS is lower when optimizing with the multi-objective approach. Finally, the payback years are higher in mono-objective optimization. With this, it can be affirmed that the multi-objective optimization has a better cost-benefit ratio than the mono-objective optimization. A significant advantage of the HYRES system, as well as being a system adaptable to different case studies, reliable and feasible as shown above, it presents the possibility of performing multi-objective optimizations that has been proven to have a best performance than the mono-objective optimization, and is not implemented in other commercial systems like HOMER. 
a) ICC comparison Click on the group you want to test

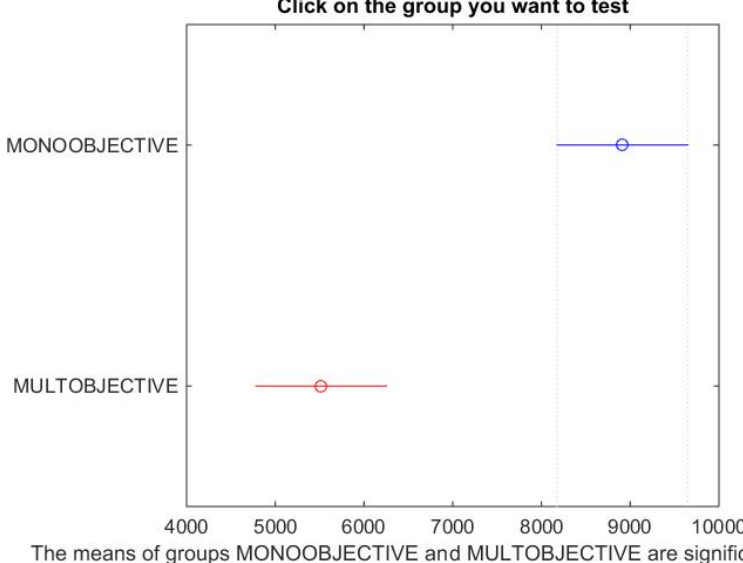

c) LPS comparison

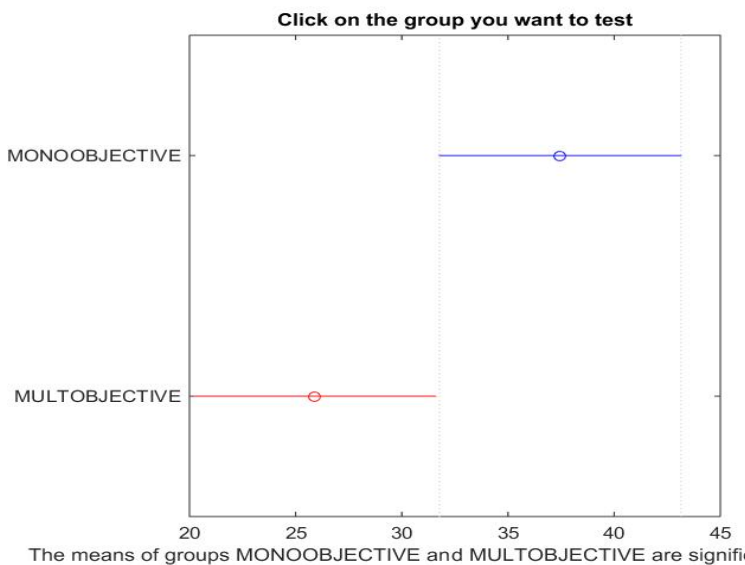

b) LCC comparison Click on the group you want to test

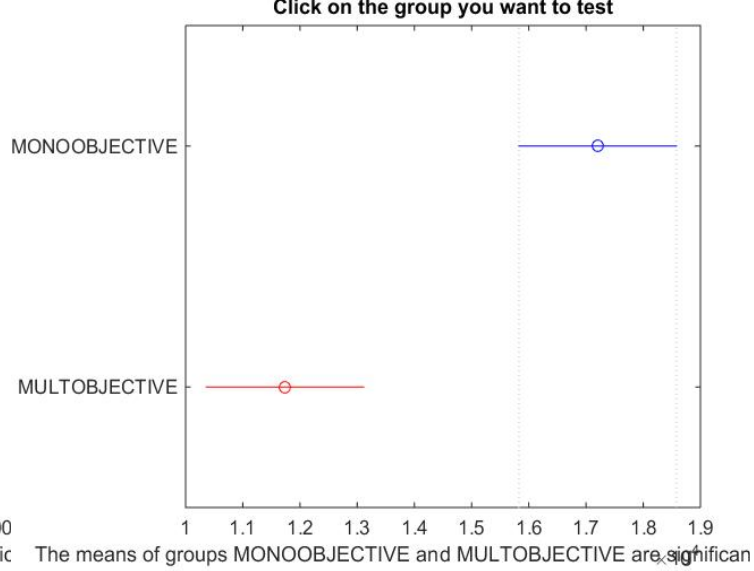

d) Reliability comparison

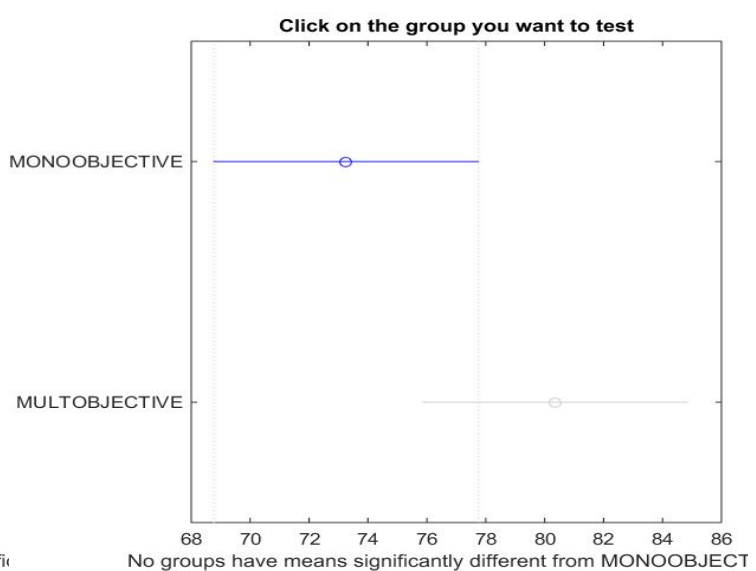

e) Payback years comparison

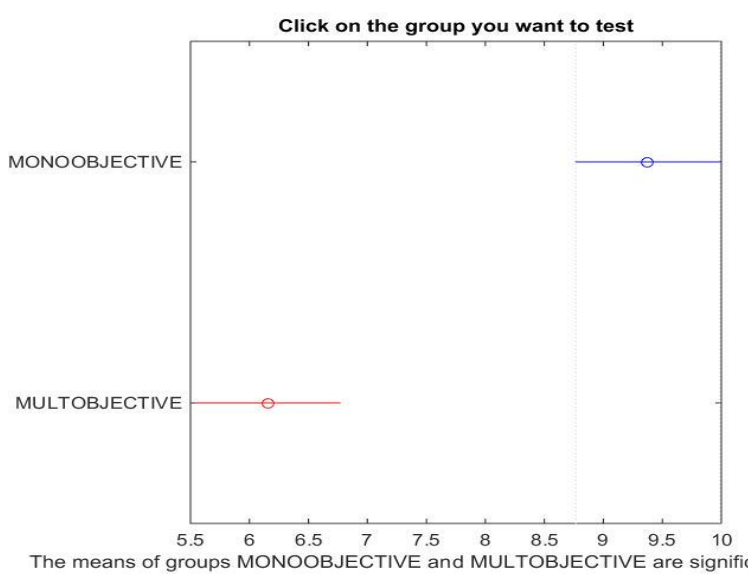

Figure 33. Comparison of the results of mono-objective and multi-objective optimization with a) ICC, b)LCC, c)LPS, d)Reliability, e)Payback years approaches. 


\section{Chapter 7}

\section{Conclusions and Future Works}

This chapter summarizes the main conclusions arisen of the analysis and discussion of the results reported in this work. The chapter also reviews the dissertation's scientific contributions and then discusses promising directions for future research and applications in certain topics in which the work of this thesis can continue. Finally, some concluding remarks are drawn.

\subsection{Conclusions}

This study could play a vital role in decision making towards sustainability. It is shown that a HRES allows the renewable energy to be used efficiently and economically, aiming to achieve the best power reliability and lowest cost. When a system has a variety of design possibilities, it becomes attractive as it allows various input parameters because the current needs vary from one case study to another in respect of climatic conditions, reliability and economic views.

HYRES, the system implemented in this thesis, has demonstrated utility, feasibility and reliability. The comparisons made with HOMER, a commercial system leader in designing microgrids has shown that HYRES has statistically accepted responses; however, HYRES system proposes additional features that make it more viable to explore different scenarios of a case study.

The main features of this system are the possibility to make multi-objective optimization by using genetic algorithms. The adaptability of the system in terms of restrictions like initial capital cost, minimum reliability, maximum area available for solar panels and wind turbines. The possibility of optimizing different reference of the system components to find the best ones for each case. Finally the response of 
the HYRES, because it shows the amount of elements that should be installed in a system.

\subsection{Main Contributions}

Motivated by the challenges mentioned in Chapter 1 , this thesis presents a system oriented to dimensioning hybrid renewable energy systems with solar photovoltaic and wind resources. The proposed system contributes with powerful tools to perform the optimization of renewable hybrid systems, through the use of genetic algorithms, which as shown in Chapter 3, are algorithms highly used for this type of studies due to their adaptive properties. The results analysis for the different case studies where the system was tested show that it has properties of utility, viability and reliability.

This thesis makes the following contributions in sizing hybrid renewable energy systems:

- An intelligent approach for sizing renewable energy systems capable to be adapted in different scenarios, with reliability, adaptability and flexibility features.

- Two reliability variables for hybrid systems, annual reliability and hourly reliability.

- To improve the cost-benefit rate of hybrid systems, it is developed a computational tool with multi-objective optimization techniques using genetic algorithms.

\subsection{Future Research and Directions}

The development of strategies focused on hybrid renewable energy systems are based in cost-benefits analysis with the main components as solar panels, wind turbines and batteries, according to the configuration of the system. In this thesis, different aspects of renewable systems were taking into account; however some 
aspects even can be improved, hence below they are present some topics to review for future works:

- Optimization approach taking into account ambient features as CO2 emissions.

- Life cycle of the elements in dependence of the usage and ambient conditions.

- Load priorization and management when the grid is not available for gridconnected systems or off grid systems.

- Include elements as load controllers, battery chargers and insulation transformers.

- Include other renewable energy systems or additional sources as generators. 


\section{References}

Al., A. et. (2017). A statistical approach for hybrid energy storage system sizing based on capacity distributions in an autonomous PV/Wind power generation system. Renewable Energy, 103, 81-93. http://doi.org/10.1016/j.renene.2016.11.024

Al Busaidi, A. S., Kazem, H. A., Al-Badi, A. H., \& Farooq Khan, M. (2016). A review of optimum sizing of hybrid PV-Wind renewable energy systems in oman. Renewable and Sustainable Energy Reviews, 53, 185-193. http://doi.org/10.1016/j.rser.2015.08.039

Alsayed, M., Cacciato, M., Scarcella, G., \& Scelba, G. (2013). Multicriteria optimal sizing of photovoltaic-wind turbine grid connected systems. IEEE Transactions on Energy Conversion, 28(2), 370-379. http://doi.org/10.1109/TEC.2013.2245669

Arabali, A., Ghofrani, M., Etezadi-Amoli, M., \& Fadali, M. S. (2014). Stochastic performance assessment and sizing for a hybrid power system of Solar/Wind/Energy Storage. IEEE Transactions on Sustainable Energy, 5(2), 363-371. http://doi.org/10.1109/TSTE.2013.2288083

Baca Urbina, G. (2007). Fundamentos de ingeniería económica (IV Edition). Mc Graw Hill. Retrieved from https://erods.files.wordpress.com/2013/02/fundamentos-de-ingenierc3adaeconc3b3mica-gabriel-baca-

urbina.pdf\%5Cnhttps://erods.files.wordpress.com/2013/02/fundamentosde-ingenierc3ada-econc3b3mica-gabriel-baca-urbina.pdf

Bayod-Rújula, Á. A., Haro-Larrodé, M. E., \& Martínez-Gracia, A. (2013). Sizing criteria of hybrid photovoltaic-wind systems with battery storage and selfconsumption considering interaction with the grid. Solar Energy, 98, 582-591. http://doi.org/10.1016/j.solener.2013.10.023

Belmili, H., Almi, M. F., Bendib, B., \& Bolouma, S. (2013). A computer program development for sizing stand-alone Photovoltaic-Wind hybrid systems. Energy Procedia, 36, 546-557. http://doi.org/10.1016/j.egypro.2013.07.063

Bilal, B. O., Sambou, V., Ndiaye, P. A., \& Kébé, C. M. F. (2013). Multi-objective Design 
of PV-Wind-Batteries Hybrid Systems by Minimizing the Annualized Cost System and the Loss of Power Supply Probability ( LPSP ), 861-868.

Bilal, F. (2013). Kurdistan Engineering Colleges and Using of Artificial Neural Network for Knowledge Representation in learning process, 3(6).

Caballero, F., Sauma, E., \& Yanine, F. (2013). Business optimal design of a gridconnected hybrid PV (photovoltaic)-wind energy system without energy storage for an Easter Island's block. Energy, 61, 248-261. http://doi.org/10.1016/j.energy.2013.08.030

danial kitchener. (n.d.). SIMULATED ANNEALING TECHNIQUES AND OVERVIEW.

Essa, E. D. S. S. . E. (2004). Norma Para el Cálculo y Diseño de Sistemas de Distribución.

González, A., Riba, J.-R., Rius, A., \& Puig, R. (2015). Optimal sizing of a hybrid gridconnected photovoltaic and wind power system. Applied Energy, 154, 752-762. http://doi.org/10.1016/j.apenergy.2015.04.105

Grouz, F., \& Sbita, L. (2014). A safe and easy methodology for design and sizing of a stand-alone hybrid PV-wind system. 2014 International Conference on Electrical Sciences and Technologies in Maghreb, CISTEM 2014. http://doi.org/10.1109/CISTEM.2014.7077043

Hosseinalizadeh, R., Shakouri G, H., Amalnick, M. S., \& Taghipour, P. (2016). Economic sizing of a hybrid (PV-WT-FC) renewable energy system (HRES) for stand-alone usages by an optimization-simulation model: Case study of Iran. Renewable and Sustainable Energy Reviews, 54, 139-150. http://doi.org/10.1016/j.rser.2015.09.046

Jana, J., Saha, H., \& Das Bhattacharya, K. (2016). A review of inverter topologies for single-phase grid-connected photovoltaic systems. Renewable and Sustainable Energy Reviews, 72(April 2015), 0-1. http://doi.org/10.1016/j.rser.2016.10.049

Kalogirou, S. A. (2013). Solar Energy Engineering (II edition, Vol. 5). Elsevier. http://doi.org/10.5597/lajam00087

Katsigiannis, Y. A., Georgilakis, P. S., \& Karapidakis, E. S. (2012). Hybrid Simulated Annealing\&\#x2013;Tabu Search Method for Optimal Sizing of Autonomous Power Systems With Renewables. Sustainable Energy, IEEE Transactions on, 
3(3), 330-338. http://doi.org/10.1109/TSTE.2012.2184840

Khare, V., Nema, S., \& Baredar, P. (2016). Solar-wind hybrid renewable energy system: A review. Renewable and Sustainable Energy Reviews, 58, 23-33. http://doi.org/10.1016/j.rser.2015.12.223

Kirthiga, M. V., \& Daniel, S. A. (2010). Optimal sizing of hybrid generators for autonomous operation of a micro-grid. 2010 IEEE 26th Convention of Electrical and Electronics Engineers in Israel, IEEEI 2010, 864-868. http://doi.org/10.1109/EEEI.2010.5662085

Ma, T., Yang, H., \& Lu, L. (2014). A feasibility study of a stand-alone hybrid solarwind-battery system for a remote island. Applied Energy, 121, 149-158. http://doi.org/10.1016/j.apenergy.2014.01.090

Mahesh, A., \& Sandhu, K. S. (2015). Hybrid wind/photovoltaic energy system developments: Critical review and findings. Renewable and Sustainable Energy Reviews, 52, 1135-1147. http://doi.org/10.1016/j.rser.2015.08.008

Maleki, A., Pourfayaz, F., \& Rosen, M. A. (2016). A novel framework for optimal design of hybrid renewable energy-based autonomous energy systems: A case study for Namin, Iran. Energy, 98, 168-180. http://doi.org/10.1016/j.energy.2015.12.133

MatWorks, I. (2015). MathWorks - Makers of MATLAB and Simulink. Retrieved April 24, 2017, from http://es.mathworks.com/index.html?s_tid=gn_logo

Mokheimer, E. M. a., Al-Sharafi, A., Habib, M. a., \& Alzaharnah, I. (2015). A New Study for Hybrid PV/Wind off-Grid Power Generation Systems with the Comparison of Results from Homer. International Journal of Green Energy, 12(5), 526-542. http://doi.org/10.1080/15435075.2013.833929

Ogunjuyigbe, A. S. O., Ayodele, T. R., \& Akinola, O. A. (2016). Optimal allocation and sizing of PV/Wind/Split-diesel/Battery hybrid energy system for minimizing life cycle cost, carbon emission and dump energy of remote residential building. Applied Energy, 171, 153-171. http://doi.org/10.1016/j.apenergy.2016.03.051

Ould Bilal, B., Sambou, V., Ndiaye, P. A., Kébé, C. M. F., \& Ndongo, M. (2013). Multiobjective design of PV-wind-batteries hybrid systems by minimizing the annualized cost system and the loss of power supply probability (LPSP). Proceedings of the IEEE International Conference on Industrial Technology, 861- 
868. http://doi.org/10.1109/ICIT.2013.6505784

Pirim, H., Bayraktar, E., \& Eksioglu, B. (2008). Tabu Search : A Comparative Study, (October), 1-29.

Pradhan, A. K., \& Kar, S. K. (2015). Modeling, Simulation and Economic analysis of Offgrid Hybrid Renewable Power System for an UnElectrified Village in Odisha. Rangnekar, S., Khare, A., Mittal, A., \& Suhane, P. (2016). Sizing and performance analysis of standalone wind-photovoltaic based hybrid energy system using ant colony optimisation. IET Renewable Power Generation, 10(7), 964-972. http://doi.org/10.1049/iet-rpg.2015.0394

S.N. Sivanandam, \& S.N. Deepa. (2008). Introduction to Genetic Algorithms. Springer. Saharia, B. J., Zaheeruddin, Manas, M., \& Ganguly, A. (2016). Optimal sizing and cost assesment of hybrid Renewable Energy Systems for Assam Engineering College. 12th IEEE International Conference Electronics, Energy, Environment, Communication, Computer, Control: (E3-C3), INDICON 2015, 1-6. http://doi.org/10.1109/INDICON.2015.7443106

Sharafi, M., ElMekkawy, T. Y., \& Bibeau, E. L. (2015). Optimal design of hybrid renewable energy systems in buildings with low to high renewable energy ratio. Renewable Energy, 83, 1026-1042. http://doi.org/10.1016/j.renene.2015.05.022

Shi, Z., Wang, R., \& Zhang, T. (2015). Multi-objective optimal design of hybrid renewable energy systems using preference-inspired coevolutionary approach. Solar Energy, 118, 96-106. http://doi.org/10.1016/j.solener.2015.03.052

Sinha, S., \& Chandel, S. S. (2015a). Review of recent trends in optimization techniques for solar photovoltaic-wind based hybrid energy systems. Renewable and Sustainable Energy Reviews, 50, 755-769. http://doi.org/10.1016/j.rser.2015.05.040

Sinha, S., \& Chandel, S. S. (2015b). Review of recent trends in optimization techniques for solar photovoltaic-wind based hybrid energy systems. Renewable and Sustainable Energy Reviews, 50, 755-769. http://doi.org/10.1016/j.rser.2015.05.040

Sinha, S., \& Chandel, S. S. (2015c). Review of recent trends in optimization techniques for solar photovoltaic - wind based hybrid energy systems. 
Renewable and Sustainable Energy Reviews, 50, 755-769. http://doi.org/10.1016/j.rser.2015.05.040

Sørensen, B. (2004). Renewable Energy (III Editio). http://doi.org/10.1016/B978-0$12-656153-1.50019-4$

Subrahmanyam, J. B. V, Alluvada, P., Bhanupriya, K., \& Shashidhar, C. (2012). Renewable Energy Systems: Development and Perspectives of a Hybrid SolarWind System. Engineering, Technology \& Applied Science Research, 2(1), 177181.

Twidell, J., \& Weir, T. (2006). Renewable Energy Resources (2nd ed.). Abingdon, UK: Taylor \& Francis. http://doi.org/10.4324/9780203478721

Wu, Z., Tazvinga, H., \& Xia, X. (2015). Demand side management of photovoltaicbattery hybrid system. Applied Energy, 148, 294-304. http://doi.org/10.1016/j.apenergy.2015.03.109

Xu, L., Ruan, X., Mao, C., Zhang, B., \& Luo, Y. (2013). An improved optimal sizing method for wind-solar-battery hybrid power system. IEEE Transactions on Sustainable Energy, $4(3)$, 774-785. http://doi.org/10.1109/TSTE.2012.2228509

Zeng, J., Li, M., Liu, J. F., Wu, J., \& Ngan, H. W. (2010). Operational Optimization of a Stand-alone Hybrid Renewable Energy Generation System based on an Improved Genetic Algorithm. Power and Energy Society General Meeting, EEE, 1-6. http://doi.org/10.1109/PES.2010.5589885

Zhou, W., Lou, C., Li, Z., Lu, L., \& Yang, H. (2010). Current status of research on optimum sizing of stand-alone hybrid solar-wind power generation systems. Applied Energy, $87(2)$ 380-389. http://doi.org/10.1016/j.apenergy.2009.08.012 


\section{APPENDIX}

The items stored in the backup references are shown in Table 8 for solar panels, Table 9 for wind turbines and Table 10 for batteries.

\begin{tabular}{|c|c|c|c|c|}
\cline { 2 - 5 } \multicolumn{1}{c|}{} & Panel 1 & Panel 2 & Panel 3 & Panel 4 \\
\hline Reference & CSun250 & LG320N1C & KD330 & HYUNDAI S280 \\
\hline Nhort circuit current [A] & 45 & 46 & 45 & 45 \\
\hline Open circuit voltage [V] & 8.81 & 10.05 & 8.79 & 38.9 \\
\hline Current at maximum power point [A] & 37.3 & 40.9 & 49.9 & 8.9 \\
\hline Voltage at maximum power point [V] & 29.9 & 9.53 & 8.15 & 31.5 \\
\hline Short circuit current coefficient \%/K & 0.045 & 0.03 & 0.06 & 0.032 \\
\hline Open circuit voltage coefficient \%/K & -0.408 & -0.28 & -0.36 & -0.32 \\
\hline Panel Dimension [m] & 1.6 & 1.64 & 1.66 & 1.640 \\
\hline Panel Dimension [m] & 0.99 & 1 & 1.32 & 988 \\
\hline Price per unit USD\$ & 160 & 340.2 & 488 & 242.55 \\
\hline Life Cycle [Years] & $\mathbf{2 5}$ & $\mathbf{2 5}$ & $\mathbf{2 5}$ & $\mathbf{2 5}$ \\
\hline
\end{tabular}

Table 8. Solar panel references as backup of the system

\begin{tabular}{|c|c|c|c|c|}
\cline { 2 - 5 } \multicolumn{1}{c|}{} & WT 1 & WT 2 & WT 3 & WT 4 \\
\hline Reference & Excel1 & Pika T701 & AIR X & Air 30 \\
\hline Rated Power [W] & 1000 & 1500 & 400 & 550 \\
\hline Power Coefficient [\%] & 0.45 & 0.45 & 0.45 & 0.45 \\
\hline Wind Speed cut in [m/s] & 2.5 & 3 & 3.6 & 3.1 \\
\hline Wind speed rated power [m/s] & 11 & 10 & 12.5 & 12.5 \\
\hline Wind speed cut out [m/s] & 54 & 66 & 49 & 35 \\
\hline Mechanical component efficiency [\%] & 1 & 0.95 & 0.95 & 0.95 \\
\hline Diameter of rotor [m] & 2.5 & 3 & 1.15 & 1.17 \\
\hline Price per unit [USD\$] & 1500 & 5295 & 849 & 795 \\
\hline Life Cycle [Years] & 25 & 10 & 25 & 12 \\
\hline
\end{tabular}

Table 9. Wind turbine references as backup of the system 


\begin{tabular}{|c|c|c|c|}
\cline { 2 - 4 } \multicolumn{1}{c|}{} & Battery 1 & Battery2 & Battery 3 \\
\hline Reference & BBOX 38 & G30-H & 8GGC21 \\
\hline Maximum current [A] & 38 & 97.6 & 182 \\
\hline Maximum voltage [V] & 12 & 12 & 12 \\
\hline Maximum depth of battery [\%] & 0.14 & 0.1 & 0.1 \\
\hline Self-discharging rate [\%] & 1 & 1 & 1 \\
\hline Battery bank efficiency charge [\%] & 94 & 96 & 96 \\
\hline Price per unit [USD\$] & 129 & 198 & 232 \\
\hline Life cycle [years] & 6 & 5 & 5 \\
\hline
\end{tabular}

Table 10. Batteries references as backup of the system 